NUMERICAL SIMULATION OF FLOW IN FLUIDIC VALVES IN

ROTATING DETONATION ENGINES

by

\author{
NANDINI GOPALAKRISHNAN \\ Presented to the Faculty of the Graduate School of \\ The University of Texas at Arlington in Partial Fulfillment \\ of the Requirements \\ for the Degree of \\ MASTER OF SCIENCE IN AEROSPACE ENGINEERING
}

THE UNIVERSITY OF TEXAS AT ARLINGTON

August 2017 
Copyright @ by Nandini Gopalakrishnan 2017

All Rights Reserved 


\section{Acknowledgements}

I would like to express my sincere gratitude to Dr. Donald Wilson, my thesis advisor, for giving me the opportunity to work with him on this research. I am grateful for his guidance, time and encouragement. I would like to thank Dr. Frank Lu and Dr. Harry Barnard for agreeing to serve on my committee and for their valuable suggestions. I want to take this opportunity to specially thank Dr. Barnard for always having had my best interests in heart, for taking interest in my research and for helping me out in every way that he has been able to.

I would like to acknowledge my lab mates Nandakumar Vijayakumar and James Peace at the ARC for giving me suggestions whenever I needed them. I am much obliged to my friend and fellow lab mate Nirmal Kumar Umapathy, who has helped me generously in every way at every stage of this research. I am thankful to all my friends at UTA who made my stay here comfortable and fun.

I am forever indebted to my mother, grandfather, uncle, aunt, brother and sisterin-law who have inspired and pushed me to dream big. Graduate studies would have been impossible without their moral and financial support. I owe this to them. I would also like to thank my new family for encouraging me to pursue my studies even after marriage. Lastly, I am grateful to God for all his blessings. 


\author{
Abstract \\ NUMERICAL SIMULATION OF FLOW IN FLUIDIC VALVES IN \\ ROTATING DETONATION ENGINES
}

Nandini Gopalakrishnan, MS

The University of Texas at Arlington, 2017

Supervising Professor: Donald Wilson

\begin{abstract}
Rotating detonation engines (RDE) have received considerable research attention in recent times for use in propulsion systems. The cycle frequency of operation of an RDE can be as high as $10,000 \mathrm{~Hz}$. Conventional mechanical valves cannot operate at such high frequencies, leading to the need for propellant injectors or valves with no moving parts. A fluidic valve is such a valve and is the focus of this study. The valve consists of an orifice connected to a constant area plenum cavity which operates at constant pressure. The fluidic valve supplies propellants to the detonation tube through the orifice. Hydrogen oxygen detonation is studied in a tube with fluidic valves. A detailed 19-step chemical reaction mechanism has been used to model detonation and the flow simulated in ANSYS Fluent. This research aims to determine the location of contact surface in the cavity and the time taken for the contact surface to leave the valve after a shock wave has passed through it. This will help us understand if the steady-state flow in the cavity is comprised of detonation products or fresh propellants.
\end{abstract}


Table of Contents

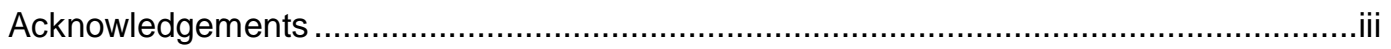

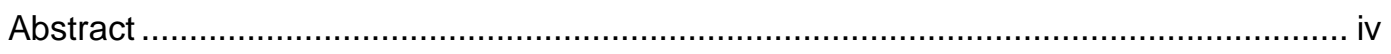

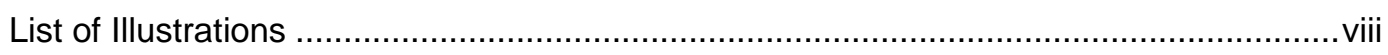

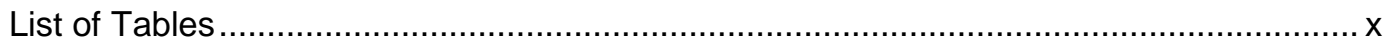

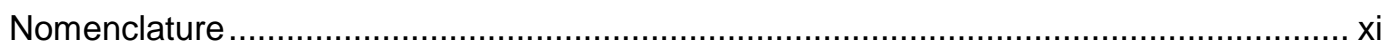

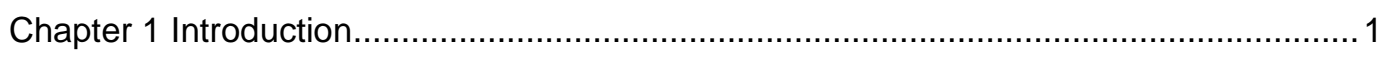

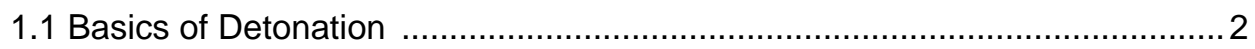

1.2 Thermodynamic Cycle and Operational Cycle ......................................... 3

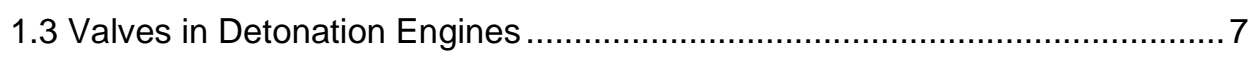

1.4 Motivation for Current Study .......................................................... 10

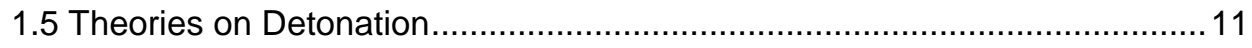

1.6 Structure of a Detonation Wave ........................................................ 16

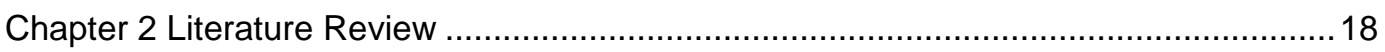

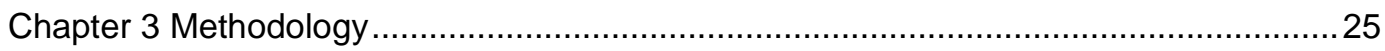

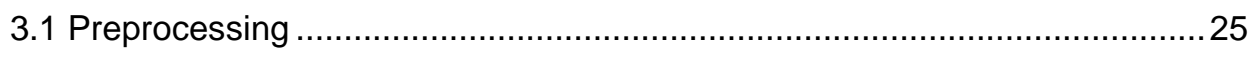

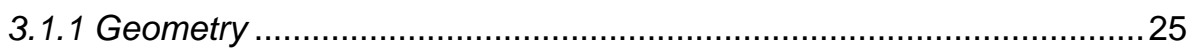

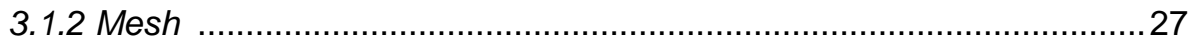

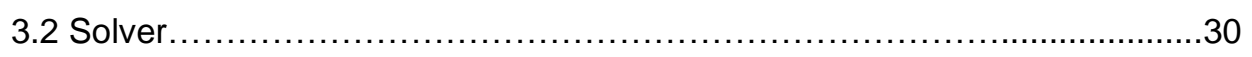

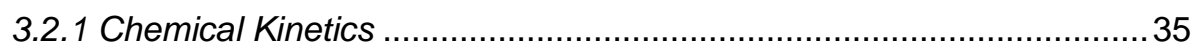

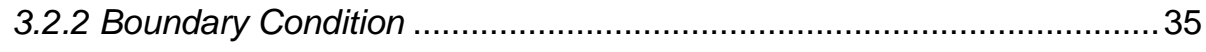

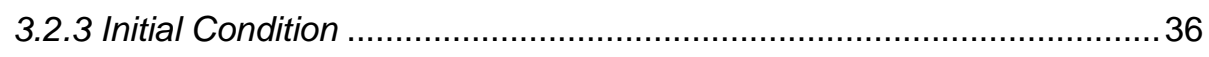

3.2.4 Computational Resource …..................................................... 38

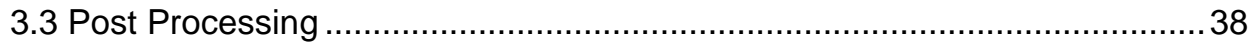

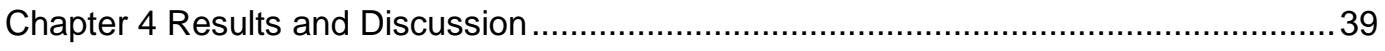




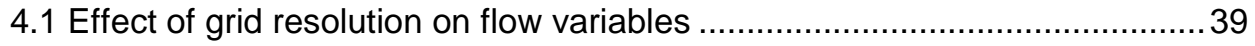

4.2 Calculation of detonation velocity ........................................................... 43

4.3 Visualization and determination of important flow parameters ................... 44

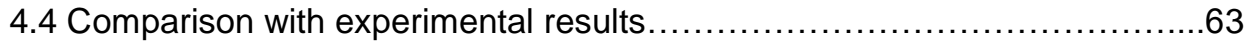

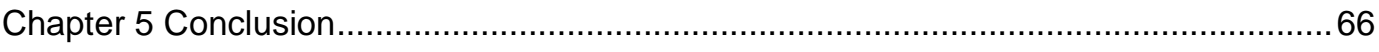

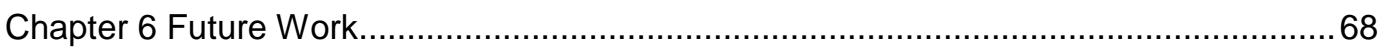

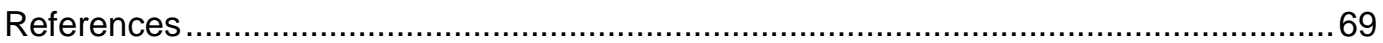


List of Illustrations

Figure 1.1 Pressure vs. Specific volume curves for different thermodynamic cycles......... 3

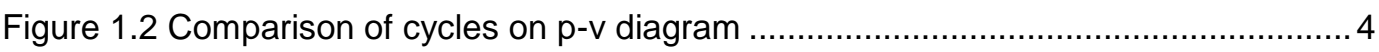

Figure 1.3 Pulsed Detonation Combustion Cycle ................................................... 5

Figure 1.4 Schematic of three-dimensional RDE .....................................................

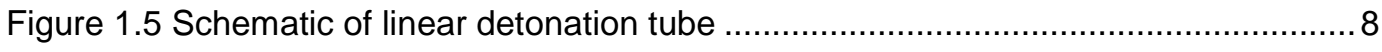

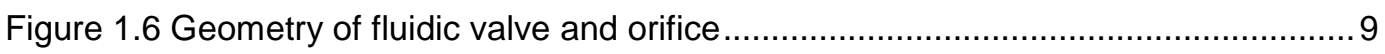

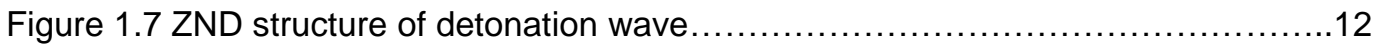

Figure 1.8 Control Volume used in the Hugoniot relations ........................................ 13

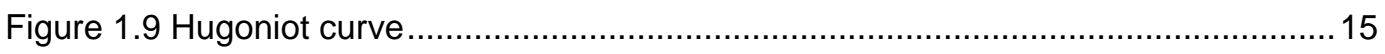

Figure 1.10 Inert and reactive Hugoniot curves ...................................................... 15

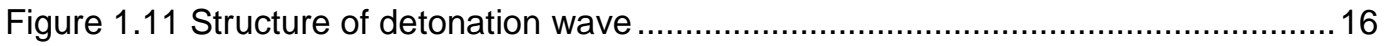

Figure 1.12 Various elements of a detonation wave .................................................. 17

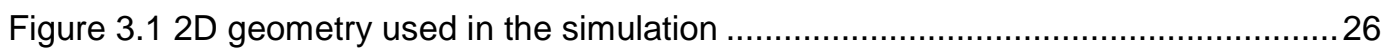

Figure 3.2 Enlarged view of the geometry to show fluidic valve ..................................26

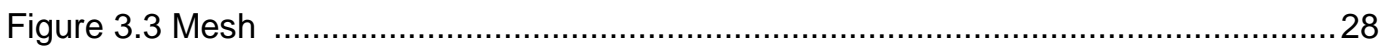

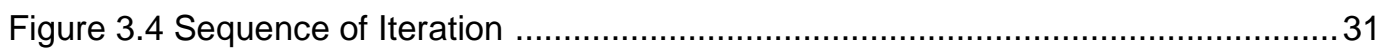

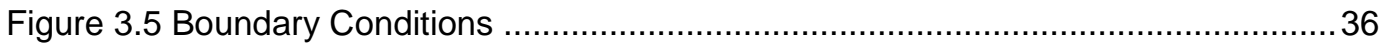

Figure 3.6 Portion of the detonation tube showing patched region ................................ 37

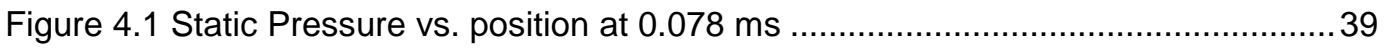

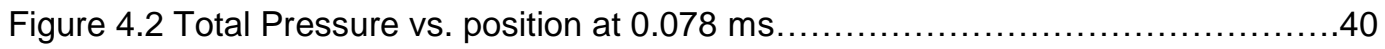

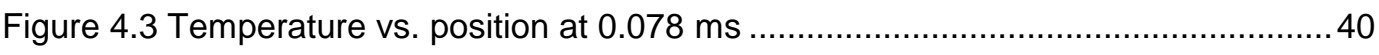

Figure 4.4 Temperature vs. position at $0.665 \mathrm{~ms}$.................................................. 41

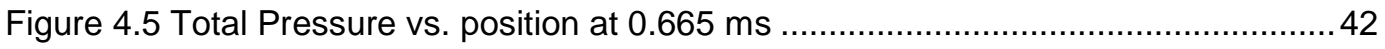

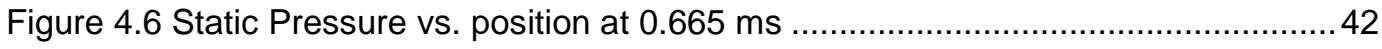




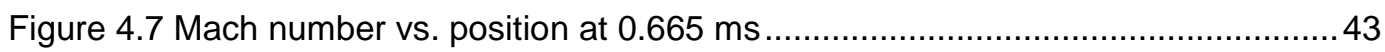

Figure 4.8 Static pressure contours at different instances............................................ 44

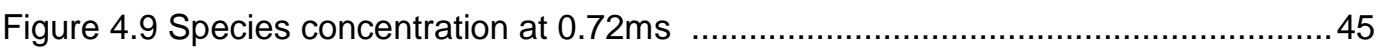

Figure 4.10 Injection of Oxygen into the tube …................................................... 46

Figure 4.11 Location of points in the plenum cavity .................................................... 47

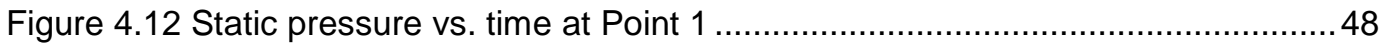

Figure 4.13 Static pressure vs. time at Point 1 showing reflections ............................... 48

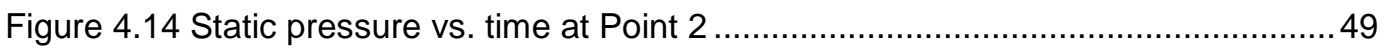

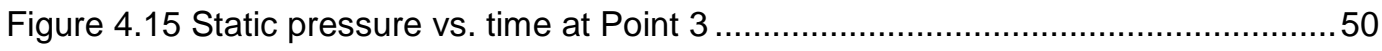

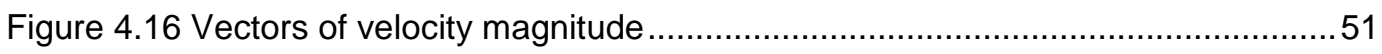

Figure 4.17 Static pressure vs. time indicating multiple reflections within the cavity .......52

Figure $4.18 \mathrm{x}$-t diagram based on static pressure showing first reflection inside the cavity.

Figure $4.19 \mathrm{x}$-t diagram based on experimental values showing multiple reflections......53

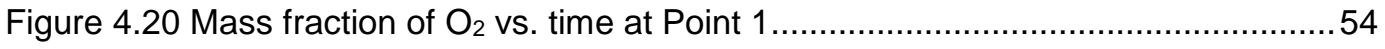

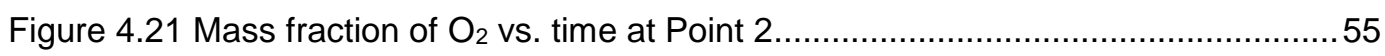

Figure 4.22 Mass fraction of $\mathrm{O}_{2}$ vs. time at Point 3................................................ 55

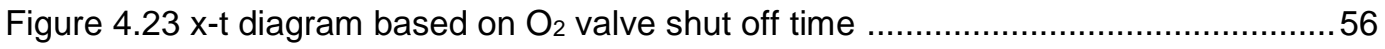

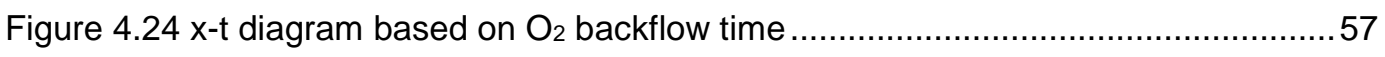

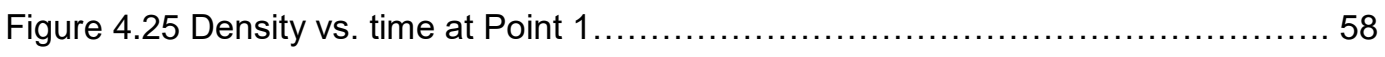

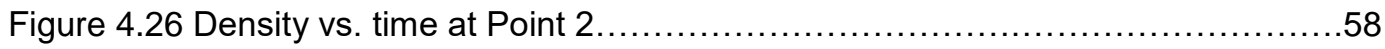

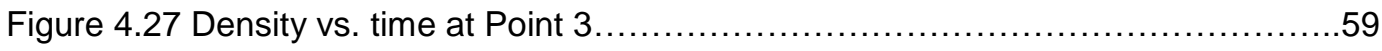

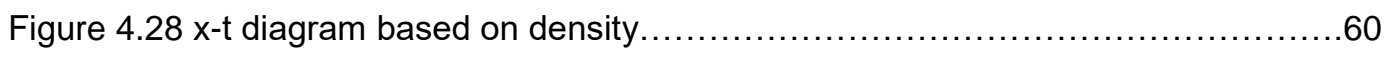

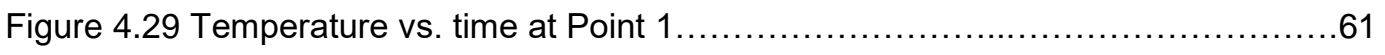

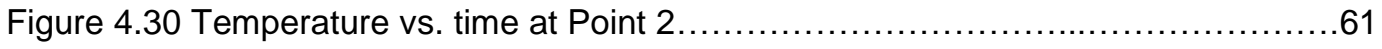


Figure 4.31 Temperature vs. time at Point 3

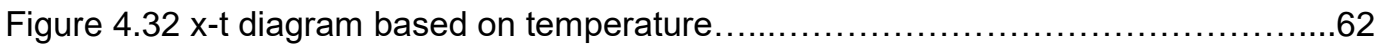

Figure 4.33 Plot of Non-dimensional pressure vs. Interruption time.....................65 


\section{List of Tables}

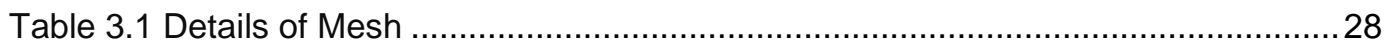

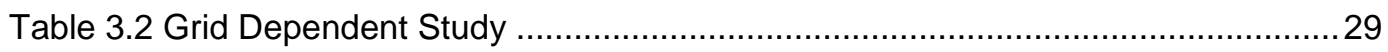

Table 3.3 Deviation of detonation parameters from CEA values ...................................29

Table 3.4 Hydrogen - Air Combustion Mechanism ...................................................... 34

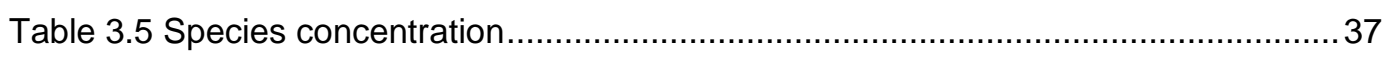

Table 4.1 Comparison with experimental results..................................64 


$\begin{array}{ll}P & =\text { Static pressure } \\ T & =\text { Temperature } \\ \rho & =\text { Density } \\ \mathrm{V} & =\text { Velocity } \\ \mathrm{u} & =\text { } \text { component of velocity vector } \\ \mathrm{V} & =\text { y component of velocity vector } \\ \mathrm{M} & =\text { Mach number } \\ \mathrm{A} & =\text { Cross sectional area } \\ \dot{m} & =\text { Mass flow rate } \\ h & =\text { Specific enthalpy } \\ q & =\text { Energy input } \\ r & =\text { Specific heat ratio } \\ \mathrm{C}_{\mathrm{p}} & =\text { Specific heat at constant pressure } \\ \mathrm{E} & =\text { Total internal energy } \\ \mathrm{Y}_{\mathrm{n}} & =\text { Species mass fraction } \\ \mathrm{R}_{\mathrm{n}} & =\text { Rate of production of Species } \\ \mathrm{K}_{\mathrm{t}, \mathrm{r}} & =\text { Forward reaction rate constant } \\ \mathrm{k}_{\mathrm{b}, \mathrm{r}} & =\text { Backward reaction rate constant } \\ \mathrm{K}_{\mathrm{r}} & =\text { Equilibrium constant } \\ \mathrm{A}_{\mathrm{r}} & =\text { Pre-exponential Arrhenius factor } \\ \mathrm{E}_{\mathrm{r}} & =\text { Activation energy for reaction } \mathrm{r} \\ \beta & =\text { Temperature exponent } \\ \mathrm{R} & =\text { Universal gas constant } \\ \mathrm{t} & =\text { Time } \\ \mathrm{Pop}_{\mathrm{op}} & =\text { Operating pressure } \\ \mathrm{M}_{\mathrm{w}} & =\text { Molecular weight } \\ \mathrm{CJ} & =\text { Chapman Jouguet state } \\ \mathrm{ZND} & =\text { Zel'dovich-von Neumann- Doring state } \\ \mathrm{PDE} & =\text { Pulse detonation engine } \\ \mathrm{RDE} & =\text { Rotary detonation engine } \\ \mathrm{F} / \mathrm{O} & =\text { Fuel-oxidizer } \\ & =\end{array}$


Chapter 1

Introduction

Propulsion technology has evolved significantly over the last century. 1903 marked the birth of the first controlled, heavier-than-air aircraft developed by the Wright brothers, making fixed wing powered flight possible [1]. Although the concept of the gas turbine engine had been around since the $18^{\text {th }}$ century, it was in 1930 that it was used for jet propulsion. Turbo engines have been modified ever since and are in use even today, powering most aircraft. The idea of ramjet engines was conceived in 1913 and it led to the development of airplanes flying at supersonic speeds. SR-71 is the fastest air breathing manned aircraft that flew at Mach 3.3. It was powered by two axial flow turbo-jet engines and used a ramjet bypass concept. High Mach limitations of ramjet engines led to an interest in scramjet engines which made hypersonic flights possible. The North American X-15 is the fastest manned aircraft powered by a rocket engine that attained Mach 6.72. Another of the $\mathrm{X}$-series planes that requires mention is the $\mathrm{X}-43 \mathrm{~A}$; a hypersonic, scramjet, unmanned aircraft that flew at Mach 9.6.

In the meanwhile, the idea of using detonation for a propulsion system was brewing parallely. Although no detonation engine has gone into production till date, the first aircraft powered by a pulsed detonation engine flew in January 2008 [2]. Detonation engines have received considerable research attention in recent times due to their potential advantages over conventional propulsion systems. The reduction in the number of compressor (and turbine) stages required due to the pressure gain with detonation combustion - leading to both cost and weight savings, higher thermal efficiency, better performance, ease of manufacture, higher thrust compared to deflagration, etc. have drawn detonation engines to possibly replace existing propulsion systems in air breathing engines and rockets. 


\subsection{Basics of Detonation}

Detonation occurs when a shock wave propagates into a reactive with the leading shock front strong enough to ignite the reactive mixture, generating a chemical reaction with heat release. This exothermic process triggers a volumetric expansion of the burned gases, forcing the shock to propagate into unburned reactants [3]. Thus, detonation is sustained by the energy released during the combustion process. The pressure rise across a detonation wave is substantial. These waves typically travel in the order of $2000 \mathrm{~ms}^{-1}$ [4], but the downstream velocity (in the wave frame of reference) is always equal to the local speed of sound, i.e., the downstream Mach number is unity. A deflagration on the other hand is a subsonic combustion wave, its speed limited to about $100 \mathrm{~ms}^{-1}[4]$. The pressure decreases or almost remains constant across such a wave. Diffusion and heat transfer play a significant role in the propagation of a deflagration while their effects are negligible in the case of detonation [5].

There are several ways by which a detonation wave can be generated. DDT (deflagration-to-detonation transition) is the most common process by which a detonation is obtained experimentally. The Shchelkin spiral is a device which aids this transition and helps reduce the length of the detonation tube. A direct initiation of the detonation wave is also possible with a sufficiently long tube having a very high energy ignition source. Alternatively, by a process known as shock-induced detonation, a shock wave from another device can be transferred to a tube of reactants causing detonation to occur immediately [6]. 


\subsection{Thermodynamic Cycle and Operational Cycle}

The operation of a conventional turbo engine is based on the Brayton cycle in which heat is added at constant pressure. Detonation based engines have been known to be modelled by Humphrey cycle wherein the fuel and oxidizer are compressed by a shock wave and heat is added to the mixture at a constant volume. The thermodynamic efficiency of a detonation engine based on Humphrey cycle is higher than a conventional turbo engine owing to the sudden compression of the $\mathrm{F} / \mathrm{O}$ mixture by the detonation waves at supersonic velocities [6]. But it was later found that other cycles represented the detonation process more closely than the Humphrey cycle. A modification of the Humphrey cycle is the Fickett Jacobs cycle which is even more efficient. In this cycle, the fuel - oxidizer mixture is compressed to the upper CJ point and heat is added through a Rayleigh heat addition process. [7]

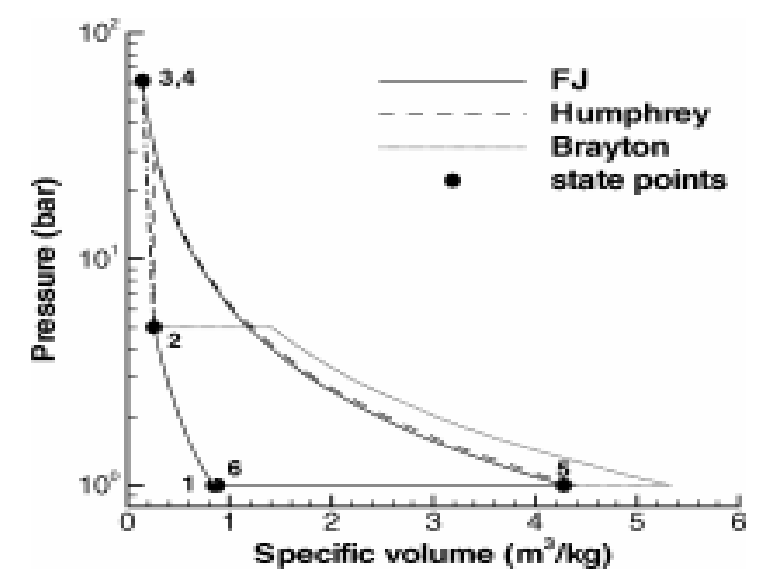

Figure 1.1: Pressure vs. Specific Volume curves for different thermodynamic cycles ${ }^{[8]}$

Another cycle that has been developed for the PDE cycle analysis is the ZND cycle [9]. As the name suggests, it follows the ZND theory. In this cycle, during the heat 
addition process, the mixture is first taken to the von-Neumann state through an inert Rankine - Hugoniot curve and then to the CJ point located on the reactive Rankine Hugoniot curve, where the reactions take place [9]. Then it follows an isentropic Taylor expansion such that final pressure is equal to the initial pressure.

The plot below compares the ZND, Brayton and Humphrey cycles. It is evident from the plot that the Humphrey cycle is closer to the Brayton cycle (hardly visible due to overlap) than the ZND cycle [9]. Thus the ZND represents a cycle of PDE more accurately than Humphrey or Fickett - Jacob cycles.

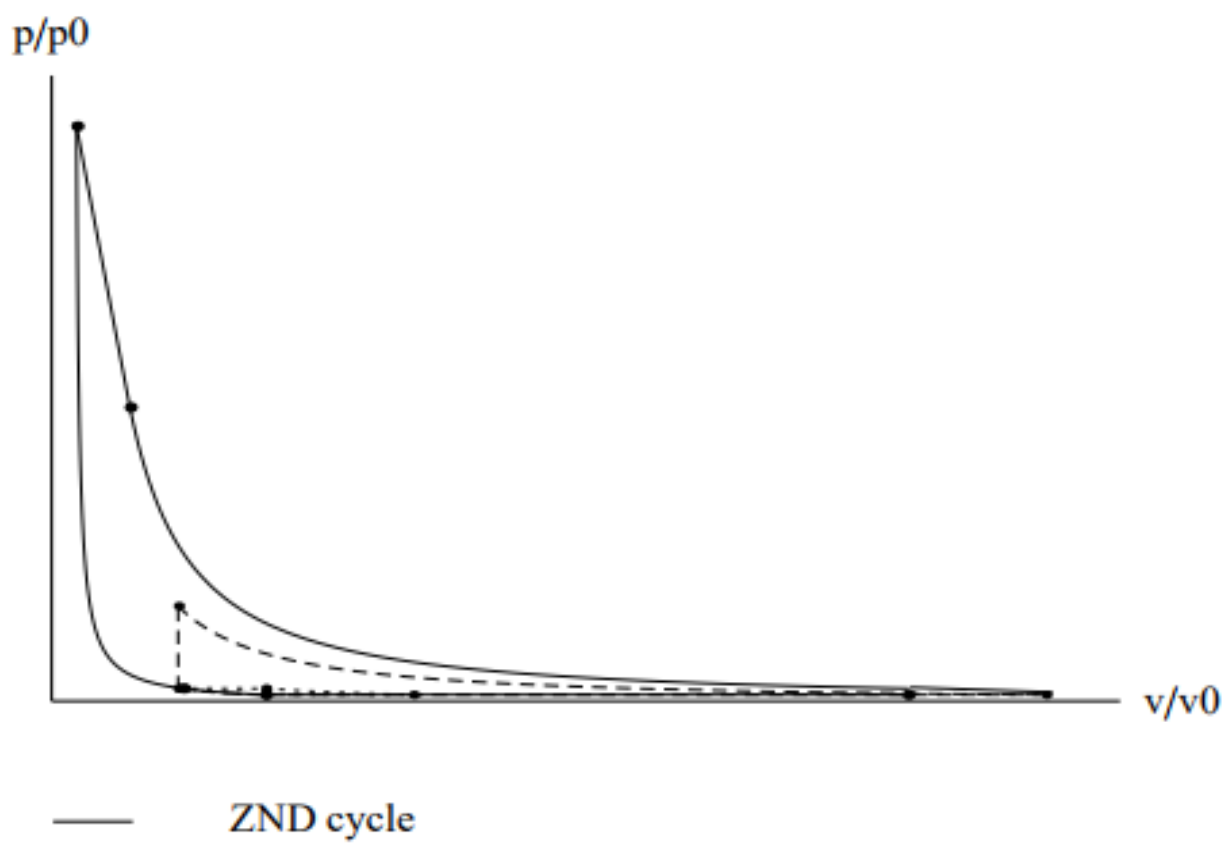

B.... Brayton cycle

---. Humphrey cycle

Figure 1.2: Comparison of cycles on $p$-v diagram [9] 
Detonation based propulsion systems are desirable because they attain higher temperatures and pressures with minimum entropy generation when compared to all other forms of combustion. Minimizing entropy maximizes thermodynamic efficiency as heat wastage is reduced.

Detonation can be of two types: pulsed detonation (unsteady) and continuous detonation (steady).

Pulsed detonation engines (PDEs) are propulsion systems that use detonation waves to combust the fuel-oxidizer mixture. These engines are usually long cylinders with one end open and the other end closed. They are unsteady/intermittent because a fresh supply of the propellant mixture has to be supplied to the engine each time a cycle of detonation is completed, i.e., the engine is pulsed between successive detonations. As a result, the thrust produced by a PDE is also pulsed.

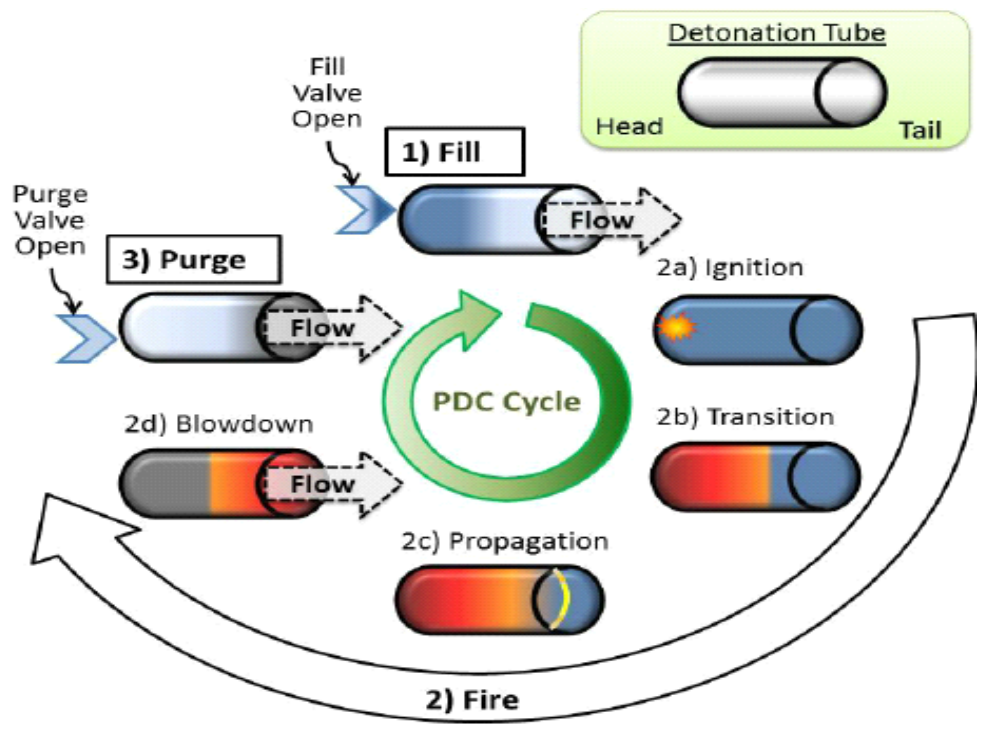

Figure 1.3: Pulsed Detonation Combustion Cycle [10] 
Figure 1.3 shows the different stages of operation of a PDE. The first step is the fill process in which the detonation tube is filled with the fuel and oxidizer. They are injected from the head end (closed end) through valves or nozzles. The second step - fire has been divided into four stages - ignition, transition, propagation and blowdown. An ignition source placed at the head end of the tube ignites a premixed fuel-oxidizer mixture. Once the deflagration transitions into a detonation, the wave propagates forward and finally, the high pressure, high temperature combustion products exit through the open or tail end of the tube in a process known as blowdown. At this stage, a purge valve opens at the head end and fills the tube with fresh air to purge any remaining combustion products. This completes one cycle, following which the tube is filled with fresh propellants again to initiate another detonation wave. Although not evident from the figure, it should be noted that, as soon as the combustion products exit the tube, an expansion wave flows back into the tube lowering the pressure inside, which aids in starting the fill process again.

Rotating detonation engines (RDEs) are a type of continuous detonation engines which have an annular ring for a combustion chamber. The detonation wave travels along the circumference of the tube continuously. Such an engine produces a continuous and quasi steady thrust. Once a detonation is initiated in an RDE, it is ideally self-sustained unlike a PDE. 


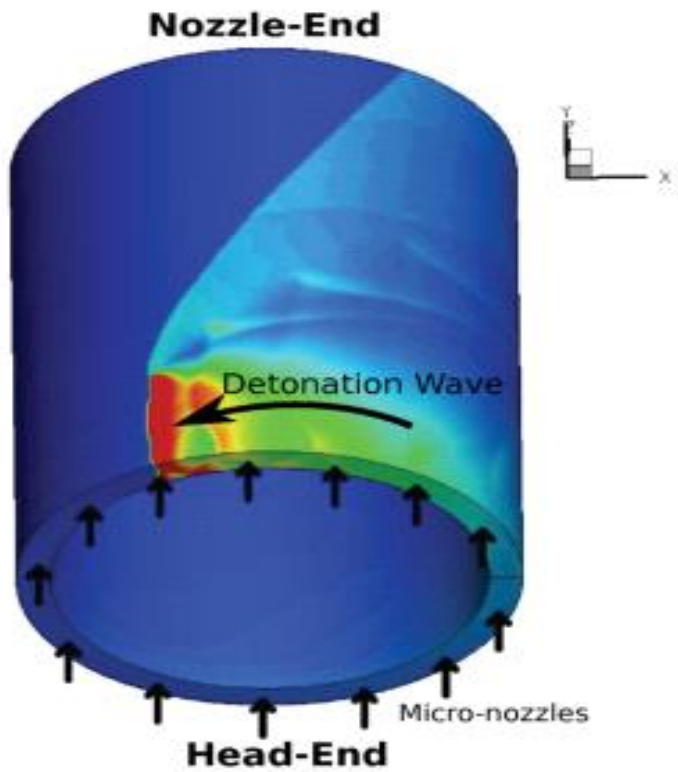

Figure 1.4: Schematic of three dimensional RDE [11]

\subsection{Valves in Detonation Engines}

The cycle frequency of operation for a PDE varies from a few hertz to about 100 $\mathrm{Hz}$. This limit could be due to the difficulty in designing a mechanical valve which is capable of opening and closing a few hundred times per second or because of a desired refilling time [12]. However, this can be overcome by using an RDE which is capable of realizing operating frequencies up to $10,000 \mathrm{~Hz}$; but these frequencies are beyond the operating limits of a conventional mechanical valve. This calls for a fluidic valve or hydrodynamic valve, which basically injects fuel and oxidizer into the tube with the valve having no moving parts. The need for a valve less mechanism led to an experiment conducted by Braun [6]. 
The experiment involved using a linear detonation tube approximately $3 \mathrm{~m}$ long with fluidic valves (FV1 \& FV2) attached radially on either side of the tube as shown in the figure below.

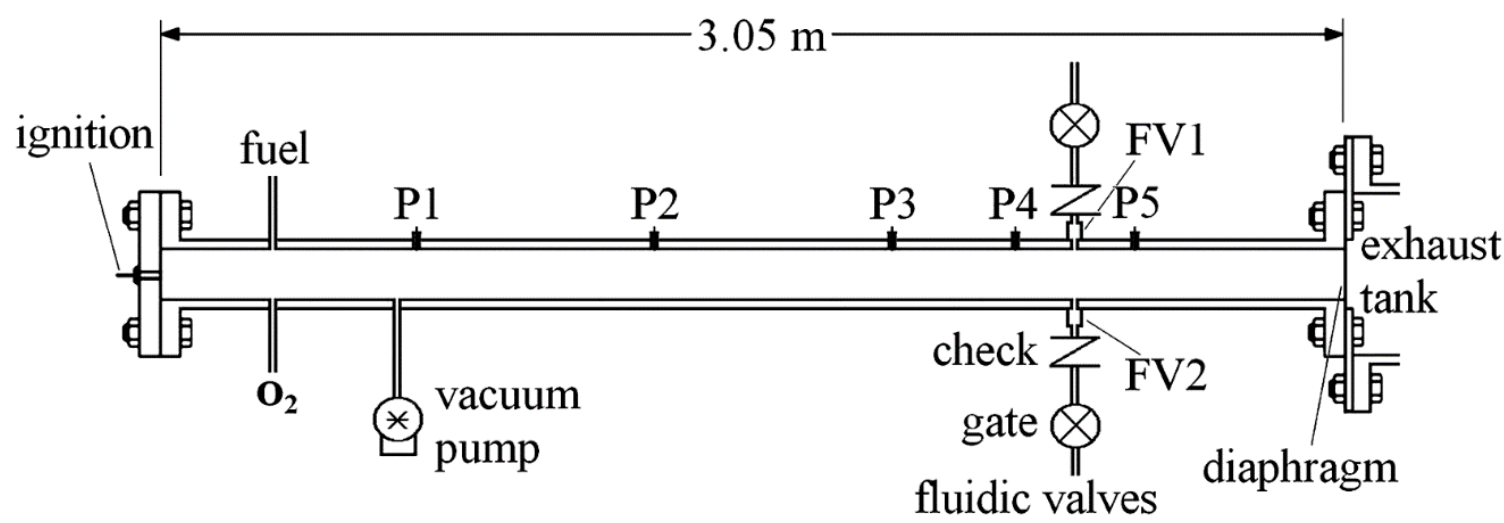

Figure 1.5: Schematic of linear detonation tube [12]

These valves serve as injectors. They consist of an orifice connected to a constant area plenum cavity which is further connected to a pipe fitting. The detailed geometry of the valve and orifices respectively are presented below. 


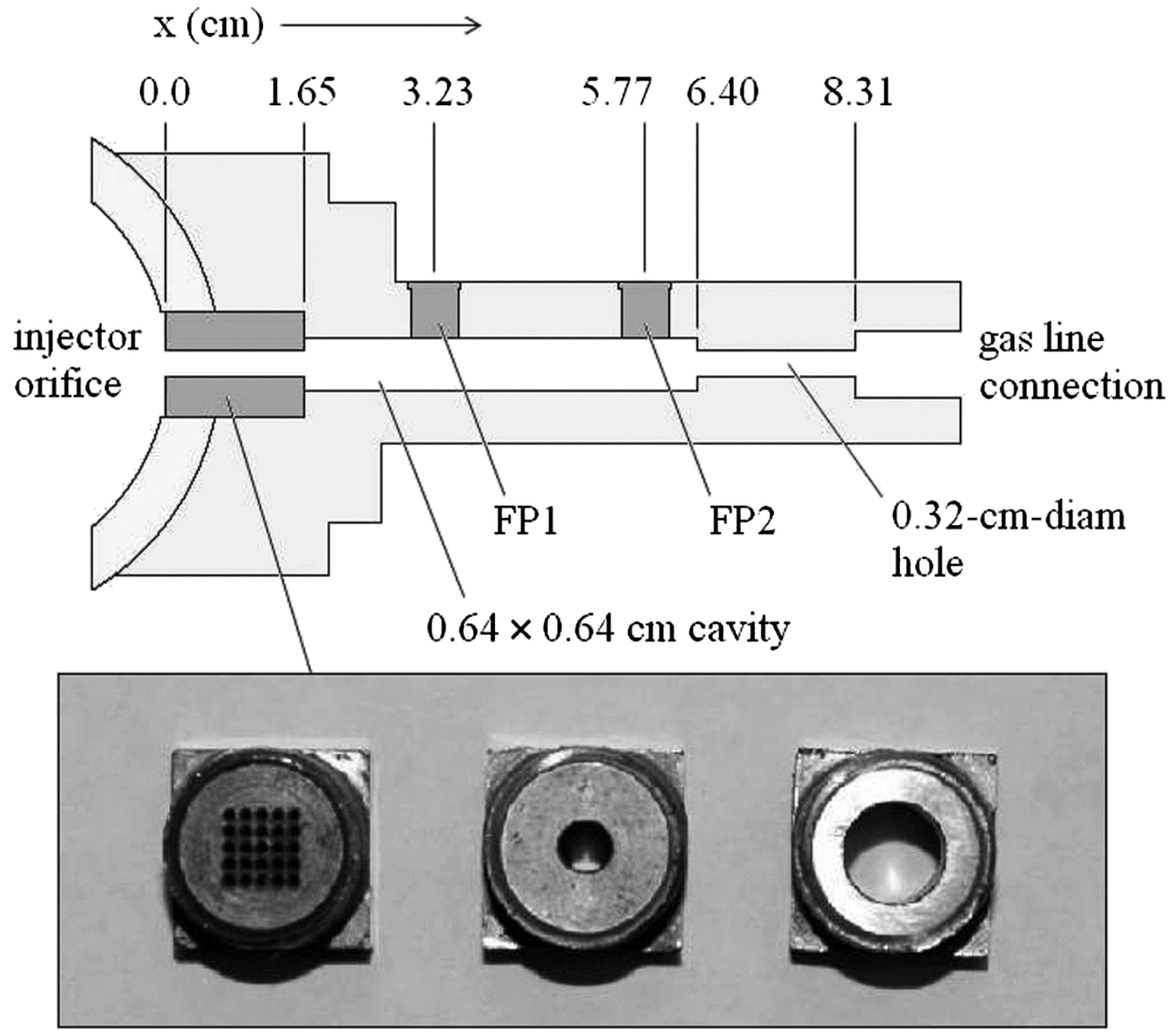

Figure 1.6: Geometry of fluidic valve and orifice [12]

Three different sets of orifices were used in the experiment. The figures from left to right are the $25 \times 0.5 \mathrm{~mm}$ orifice array, the $0.32 \mathrm{~cm}$ diameter orifice and the $0.64 \mathrm{~cm}$ diameter orifice. With the $0.64 \mathrm{~cm}$ diameter orifice, the cross-sectional area is same as that of the plenum cavity. The area is reduced upstream of the cavity to connect to the high pressure gas connection fitting. The fluidic valve uses its supply pressure to inject fuel and oxidizer into the detonation tube and thus has no moving parts. 
The detonation tube is first filled with fuel and oxidizer, and maintained at 1 atm pressure. The plenum cavity always operates at a constant pressure which is much higher than the pressure in the main tube. It is due to this pressure difference that the propellant flows into the tube. At this point, an automotive ignition source placed at the head end ignites the mixture in the tube initiating a detonation wave. It should be noted that when there is flow from the cavity into the tube, the valve is in the open position. As the detonation wave propagates and passes over the injector orifice, the high pressure combustion products flow into the cavity. This reverses the propellant flow and shuts the valve off. As the shock wave travels in the cavity, it hits the wall ahead of it and reflects back. The appearance of a Taylor rarefaction wave in the main tube causes resumption of flow into the tube from the cavity. Eventually, the pressure in the cavity returns to its steady state value, thereby turning the valve on again.

In order to have a successful RDE, it is crucial to reduce the time during which the valve is shut off and to minimize the backflow condition in the cavity. This implies that pressure in the cavity has to return to its original steady-state value as quickly as possible.

The fluidic valve mechanism was tested and validated using injector orifices with different geometries, by varying the steady-state injection pressure, by using various combinations of fuel and oxidizer in the tube, and by varying the propellants in the cavity. The primary concern in each of these tests was the time taken to return to steady state cavity pressure [6].

\subsection{Motivation for Current Study}

The experiment discussed above successfully proved that a fluidic valve capable of refueling between successive detonation wave fronts was both possible and functional 
[12]. However this work does not tell us precisely when the steady-state flow of fresh propellants is resumed. Using pressure transducers, it is not possible to experimentally determine the location of the contact surface in the cavity or the time it takes for the contact surface to leave the valve.

This calls for further investigation using computational methods which leads to the motivation of this study. The current research involves using a computational tool Fluent to simulate flow in a detonation tube with fluidic valves, having the same physics setup as the experiment.

\subsection{Theories on Detonation}

Chapman and Jouguet were one of the first to propose theories on detonations. The CJ theory is applicable to infinitesimally thin detonations and it assumes that the chemical reactions occur within the shock itself. The $\mathrm{CJ}$ condition says that behind the shock front or at the end of the reaction zone, where the chemical reaction has come to an end and the products have attained equilibrium, there exists a sonic region $(M=1)$. This means that the velocity of the gaseous products with respect to the detonation wave front is equal to the local speed of sound. This is the minimum speed at which a detonation wave travels and is known as the Chapman-Jouguet velocity.

The more recent and commonly accepted theory on detonations is the ZND theory, named after Zeldovich, Von Neumann and D"oring [13 -15]. This theory suggests that based on the composition of the reactants, a finite rate chemical reaction occurs behind the shock wave, suggesting the existence of a separate reaction zone. According to the ZND model, the reactant mixture is first compressed by the shock wave to a very high value of pressure known as the von-Neumann spike, following which the chemical reaction begins. At the end of the reaction, the products attain equilibrium and are at the 
Chapman-Jouguet state. Depending on the chemical kinetics, an induction zone may or may not be present between the leading shock and the reaction zone [16].

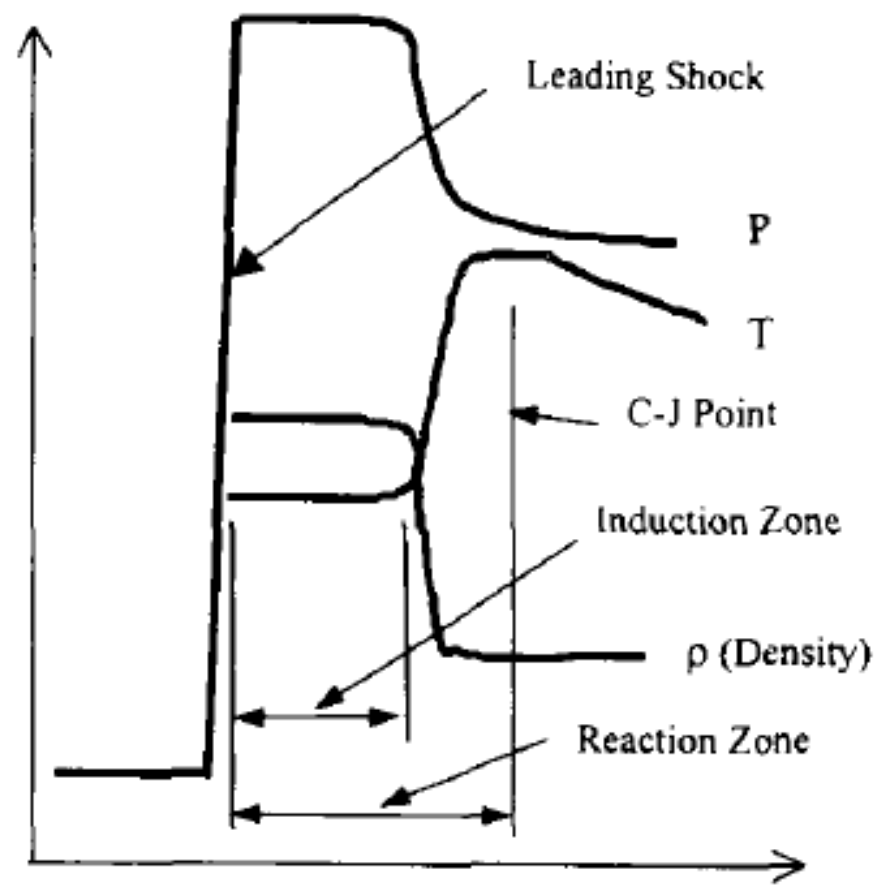

Figure 1.7: ZND structure of detonation wave [17]

The CJ theory can be explained with the help of the Rankine-Hugoniot curve. The Hugoniot relations relate thermodynamic quantities upstream and downstream of a shock wave. These relations follow simple mass, momentum and energy conservation equations. In order to arrive at the Hugoniot equations, the following assumptions are made - flow is steady, inviscid, adiabatic, one dimensional, flowing through a constant area duct, follows ideal gas behavior and neglects body forces. 


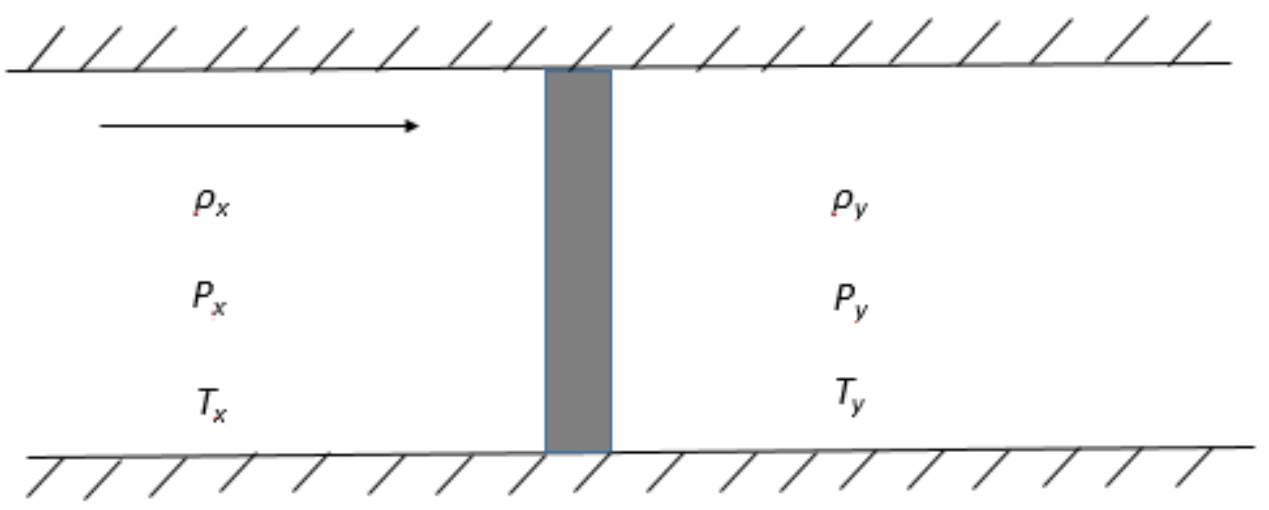

Figure 1.8: Control volume used in the Hugoniot relations

The three Hugoniot relations are:

$\rho_{x} v_{x}=\rho_{y} v_{y}=\dot{m} / A$

$P_{x}+\rho_{x} v_{x}^{2}=P_{y}+\rho_{y} v_{y}^{2}$

$h_{x}+\frac{v_{x}^{2}}{2}=h_{y}+\frac{v_{y}^{2}}{2}$

While these equations are written for a shock under adiabatic conditions, heat is released in a detonation due to chemical reactions and this heat release has to be included in the energy equation.

$h_{x}+\frac{v_{x}^{2}}{2}+q=h_{y}+\frac{v_{y}^{2}}{2}$ 
We arrive at the Rankine - Hugoniot relation by writing enthalpy per unit mass in the above equation in terms of specific heat, pressure in terms of equation of state, and combining equations (1) and (2) and substituting into (4).

$C_{p, y} T_{y}-C_{p, x} T_{x}=q+\frac{1}{2}\left(\frac{1}{\rho x}+\frac{1}{\rho y}\right)\left(P_{y}-P_{x}\right)$

If specific heat is assumed to be constant, then the Rankine - Hugoniot relation can be written as

$q=\frac{r}{r-1}\left(\frac{P y}{\rho y}-\frac{P x}{\rho x}\right)-\frac{1}{2}\left(P_{y}-P_{x}\right)\left(\frac{1}{\rho x}+\frac{1}{\rho y}\right)$

The Hugoniot curve is a plot of pressure versus specific volume (Figure 1.9 \& Figure 1.10). It is the locus of all possible solutions of the Hugoniot relations. The Rayleigh line is used to identify regions of deflagration and detonation on the Hugoniot curve. The Rayleigh line is drawn from the origin (known values of pressure and specific volume) and is tangential to the Hugoniot curve at two points known as the Chapman Jouguet points. The upper CJ point is located on the detonation branch and denotes the existence of a stable detonation while the lower CJ point is on the deflagration branch and it is the point at which a stable deflagration exists.

Region 1 of the Hugoniot curve corresponds to strong detonations with flow going from supersonic to subsonic conditions. Region 2 corresponds to weak detonations and the gas velocity remains supersonic throughout. Region 3 is physically impossible to attain as it does not satisfy the Rankine Hugoniot relations. Regions 4 and 5 are solutions of the Hugoniot curve corresponding to deflagrations. 


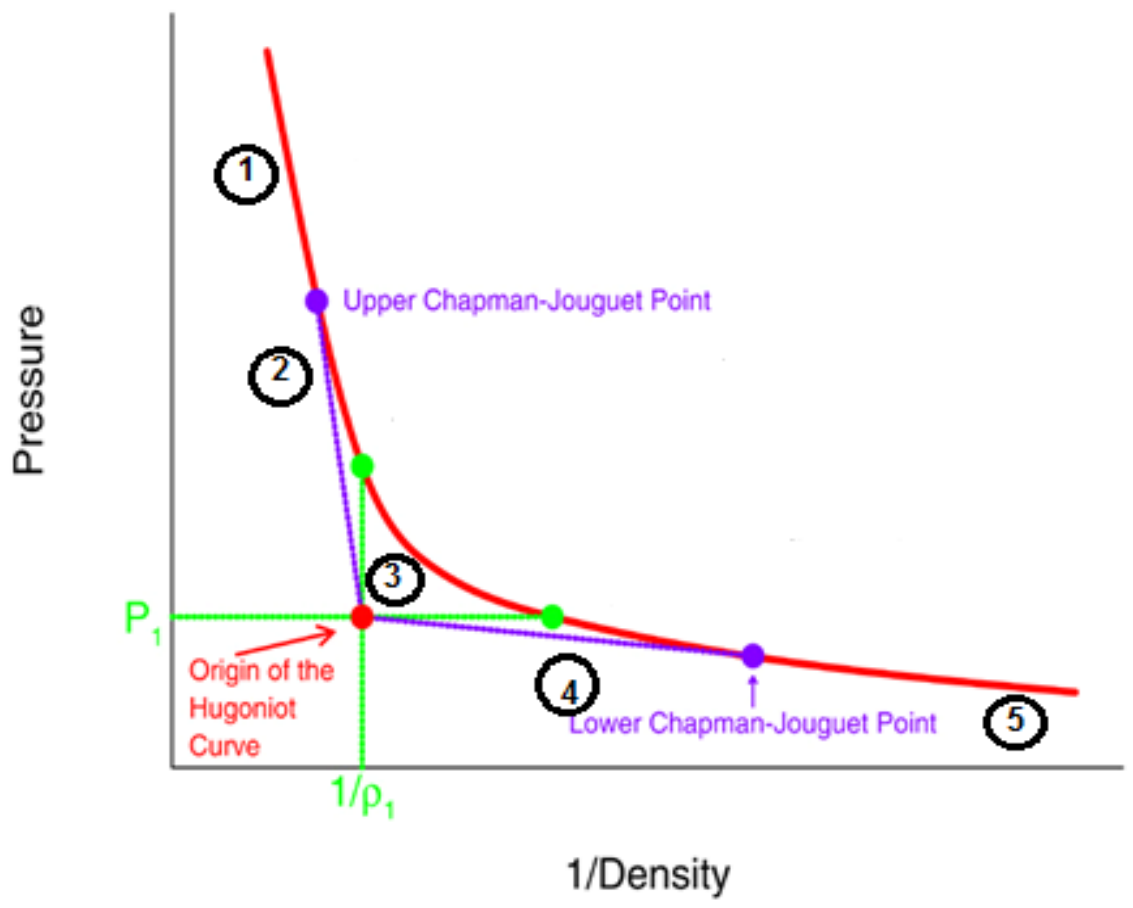

Figure 1.9: Hugoniot curve [18]

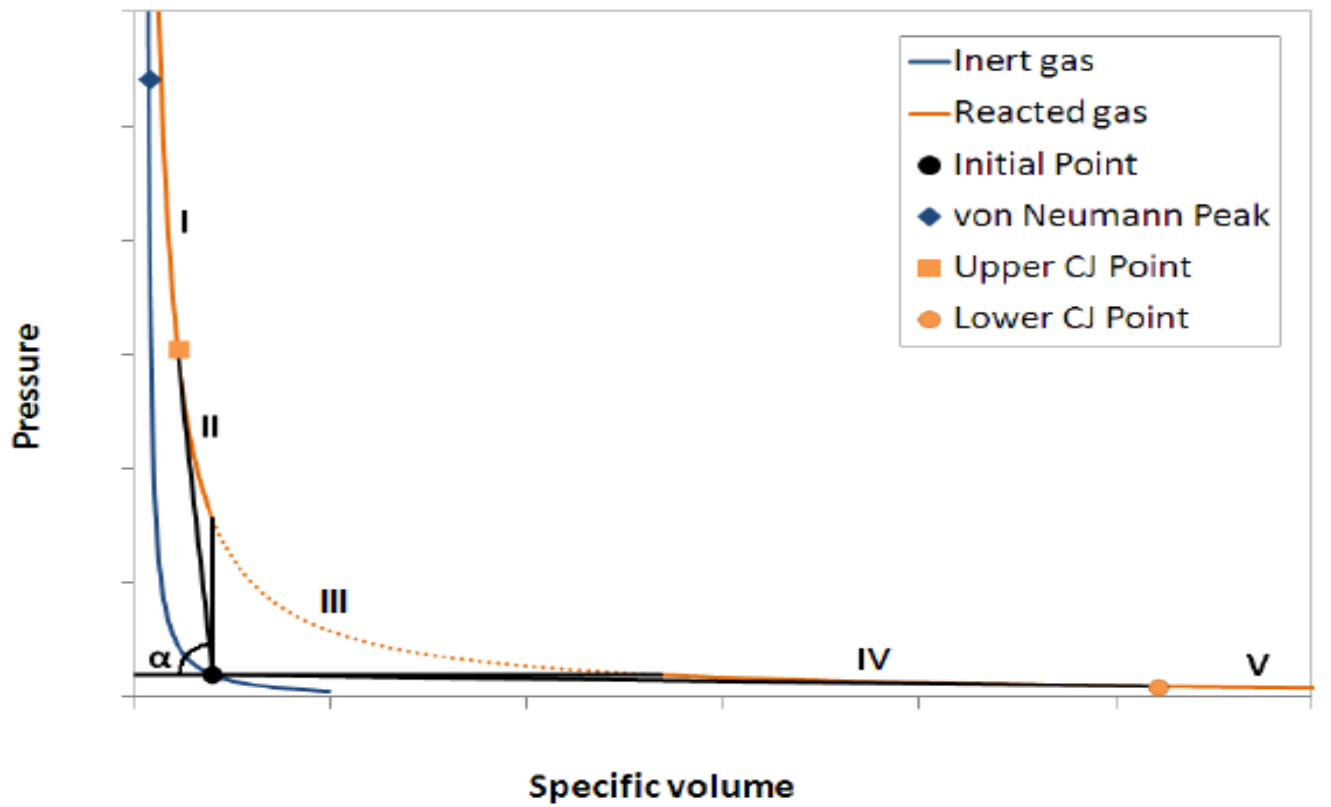

Figure 1.10: Inert and reactive Hugoniot curves [6] 


\subsection{Structure of a Detonation Wave}

Both CJ and ZND theories idealize detonation as a one-dimensional wave satisfying the reactive Euler equations. But, in reality, denotation wave has a complex multi-dimensional wave structure. The figure below clearly shows the structure of detonation, obtained from a hydrogen - oxygen detonation (first two in the presence of Argon and the last two in the presence of Nitrogen) experiment.
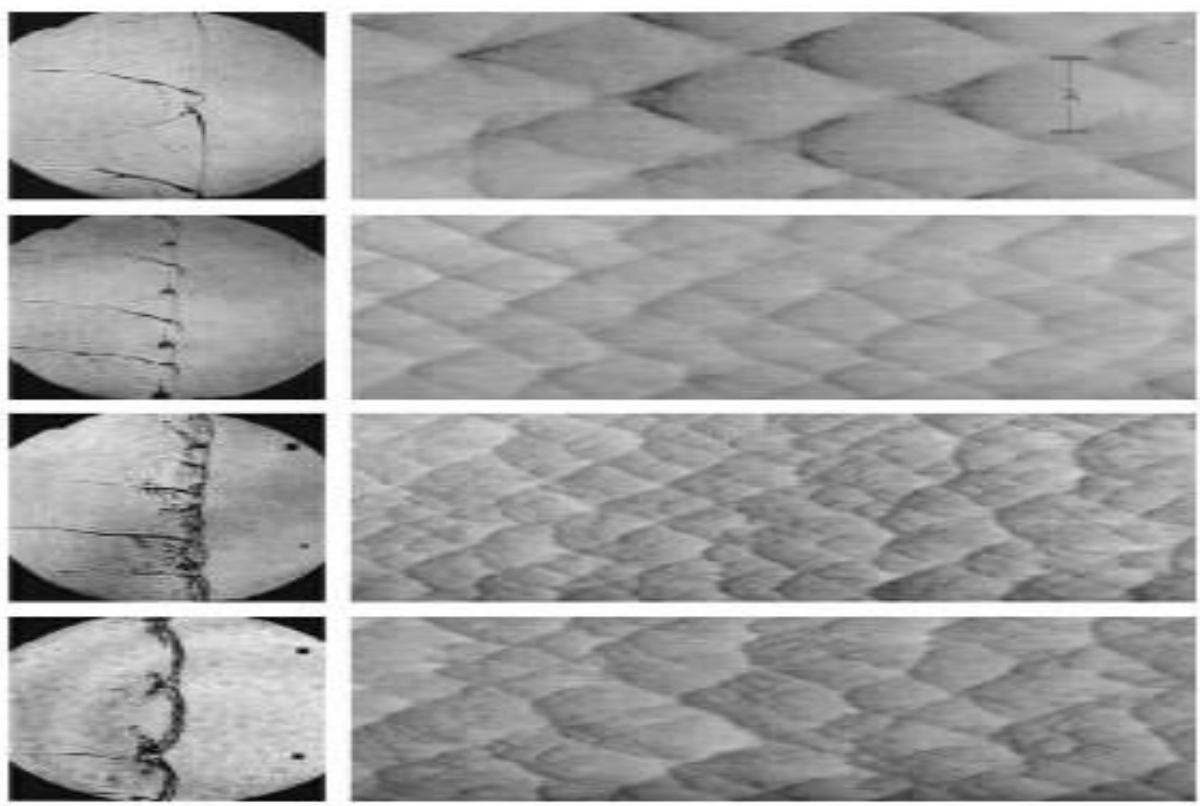

Figure 1.11: Structure of detonation wave ${ }^{[19]}$

The wave has a cellular structure and it consists of leading shock waves, transverse waves and triple points. Triple point is the point of intersection of the leading shock front and transverse waves. The cell width (lambda) is related to the length of the 
induction zone.

These patterns are observed experimentally with the help of a soot mixture placed inside the detonation tube. As the wave passes by, the triple points and thus the cellular structure get imprinted on the soot foil.

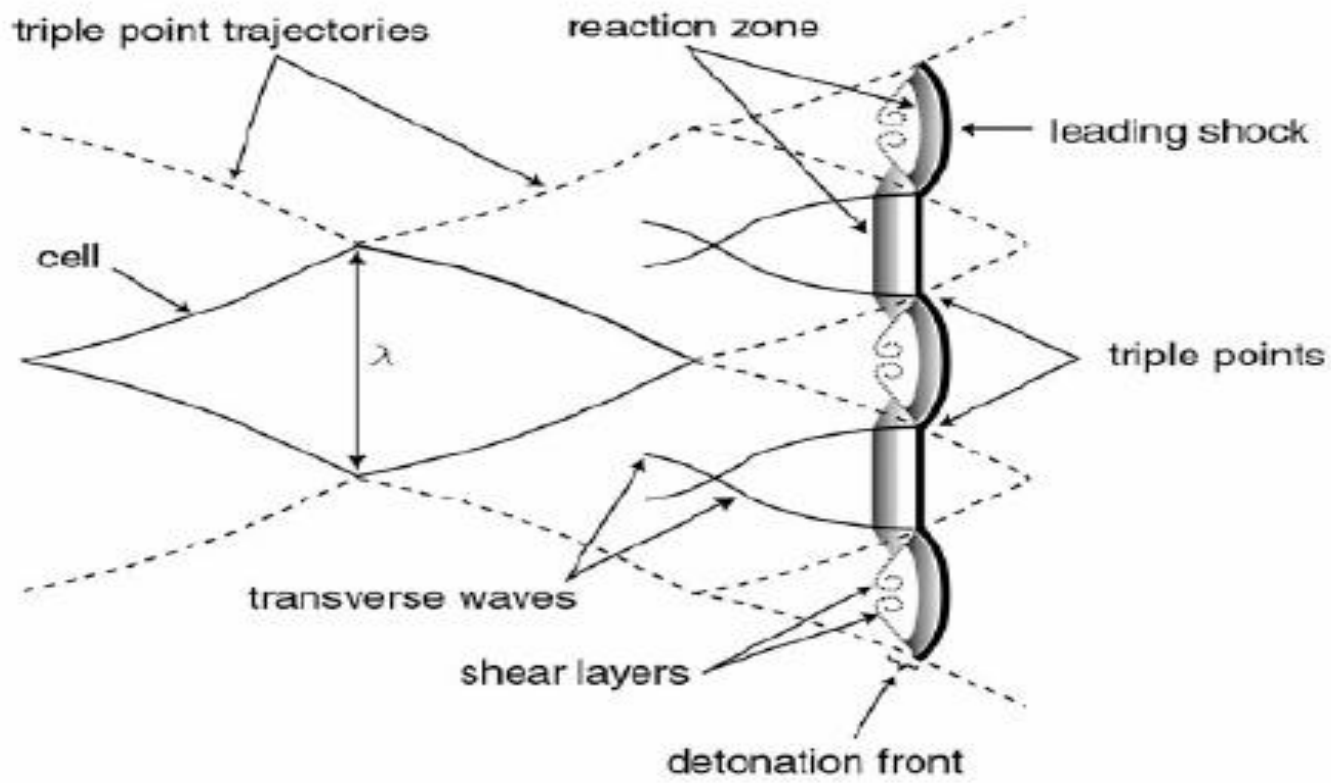

Figure 1.12: Various elements of a detonation wave [19] 
Chapter 2

\section{Literature Review}

Fickett \& Wood [20] studied the stability of one-dimensional detonations based on the numerical solutions of Erpenbeck [21]. They considered an idealized single irreversible chemical reaction, obeying the Arrhenius reaction rate law. Detonation was initiated in the channel by a shock generated by a piston located at the head end. They identified a standard mesh size for their problem, characterized as having about 10 fluid elements along a line between the leading shock front and the point of half reaction. This size was reduced by a factor of 2 for studying steady and unsteady cases. They found out that errors in flow variables due to this mesh spacing were only less than $1 \%$. Their results revealed that, in steady-state solutions that were unstable, the shock pressure oscillated about the steady-state value with peak pressure almost $50 \%$ higher. The findings were in good agreement with Erpenbeck's linearized stability analysis of steady detonations.

Oran et al. [22] studied two-dimensional $\mathrm{H}_{2}-\mathrm{O}_{2}-\mathrm{Ar}$ detonation using a detailed chemical reaction model. They could identify cellular structures in their simulations and they studied the energy release pattern in a detonation cell. Their results revealed that, in addition to the primary energy release behind the Mach stem, a secondary energy release started at about two-thirds of the way into the cell. The numerical method used was a version of the Flux-Corrected Transport algorithm (FCT), LCPFCT is an explicit, finite volume algorithm used to solve one-dimensional continuity equations with source terms. Stoichiometric hydrogen - oxygen gas mixture diluted with $70 \%$ argon at an initial pressure of $6.67 \mathrm{kPa}$ and temperature of $298 \mathrm{~K}$ was used to model detonation. Detonation was initiated on a one-dimensional grid and allowed to propagate until the mean velocity reached the $\mathrm{CJ}$ value. The one-dimensional solution was then placed on a 
two-dimensional grid and disturbed so as to generate transverse waves. The disturbance was created by placing a region of unreacted reactive gas behind the detonation front. The authors raise an important concern about how most studies compute detonation based on reactive Euler equations. They discuss whether it is necessary to use a Navier Stokes solution to model detonation and address the importance of turbulence in describing the detonation structure.

Sharpe and Falle [23] numerically simulated one-dimensional, time dependent detonations using a second-order Godunov scheme. They also performed a stability analysis using Sharpe's [24] normal mode approach to linear stability. Their analysis predicted final frequency of oscillations well for unstable detonations. However, they reported that at very high activation temperatures, detonation propagated as a series of explosions which was quite conflicting with He \& Lee's [25] findings. Their simulations showed that in such cases, detonation dies out and remains at a low pressure for a long time. Sharpe and Falle also observed that at large activation temperatures, large pockets of partly burned fuel were formed. This phenomenon was previously seen only in twodimensional detonations. They noted that the diamond or cellular structure of detonations could be observed in tubes whose width was not much larger than its reaction length scale.

Kailasanath and Patnaik [26] present a study on the effects of outflow boundary conditions and initial conditions on the flow field and performance of PDEs. They developed a code that could compute three dimensional, multi species reactive flow in simple geometries and allow inclusion of species diffusion, heat loss due to radiation and thermal conduction. A one dimensional, stoichiometric hydrogen - air detonation in a 20 $\mathrm{cm}$ long tube with one end closed and the other open is considered. They mention 
different ways of initiating detonation and highlight the difficulties in choosing the right initial conditions. For their study, the first few cells of the tube are patched with high pressure $(90 \mathrm{~atm})$ and high temperature $(3000 \mathrm{~K})$ detonation products at the $\mathrm{CJ}$ plane conditions. The grid size used was $0.002 \mathrm{~cm}$. At the exit, a pressure relaxation boundary condition is imposed such that the pressure reduces to ambient value at a fixed distance. In a typical detonation problem, the downstream boundary condition does not affect the flow, but once the flow begins to leave the tube, the method of specifying pressure relaxation will affect the flow. If the outflow pressure is set to ambient value, i.e, a relaxation length of $0 \mathrm{~cm}$, the high pressure combustion products will have to drop to ambient pressure rapidly, causing the pressure at the head end to drop to values lower than the ambient one. This results in the burned products being drawn back into the tube. However, they observed that if the products had to reach ambient value only gradually (relaxation length of $20 \mathrm{~cm}$ was studied), then the head end pressure would remain significantly above the ambient value. They found that the relaxation of pressure at the exit of the tube had a crucial role in determining the specific impulse, the more gradual the relaxation, the higher the specific impulse.

Hsu and Jemcov [27] studied the effects of finite rate simplified chemical mechanisms on detonation in a pre-mixed hydrogen - air mixture. They also focused on capturing the detonation wave structure - the Von Neumann spike, induction zone and C$\mathrm{J}$ point in particular. Comparison is made between one step, two step, 8 step and 32 step chemical mechanisms. They mention that the one and two step models have Arrhenius parameters that are functions of equivalence ratio while the 8 step and 32 step models are independent of the richness of the mixture. Commercial CFD Fluent 5 is used for the simulations. Flow is assumed to be inviscid as they find turbulence to play a minimum role in detonation propagation in shock tubes. On performing grid convergence checks, 
no major difference is found between $1 \mathrm{~mm}$ and $0.25 \mathrm{~mm}$ grid size. Their results indicate that the induction zone proposed by ZND theory doesn't exist in matured self-sustained detonations. A reaction zone smeared with the leading shock is required to support a self-sustained detonation. The reaction zone is even more closely coalesced with the leading shock for richer mixtures.

Law et al. [28] emphasize the need for comprehensive chemical kinetics to model detonation in their paper. The inadequacy of one step global reaction is explained and the subsequent steps towards developing detailed kinetic mechanisms are presented. They discuss the issue of comprehensiveness of a mechanism - a mechanism is considered adequate only if it can represent all relevant chemical responses over a wide range of system variations that may occur.

Yungster \& Radhakrishnan [29] analyzed the structure and stability of onedimensional detonations in an ethylene - air mixture using a detailed 36 step chemical reaction. Different equivalence ratios, pressures and degrees of overdrive were considered. A multi-level dynamically adaptive grid was used to maintain high numerical resolution. They observed that the detonation wave structure changed with grid spacing. Their study involved finding whether 1D pulsating detonations were observed only for hydrogen - air mixtures or whether the instabilities could be extended to other fuels as well. Their approach was quite different as it eliminated the need for steady state ZND solutions. The results from the various parametric studies conducted were as follows. The structure of detonation for lean mixtures was similar to that of stoichiometric mixtures. It was observed that detonations propagate in a high frequency -low amplitude mode for lean to slightly rich mixtures and in a low frequency - high amplitude mode for richer mixtures. For low driver pressure ratios $(70-100)$, the detonation wave displayed 
short period oscillations but transitioned to long period modes with time. For ratios between 150 and 250, only short period mode was observed and for pressure ratios greater than 275 , detonation began to stabilize. These trends were observed by Bourlioux et al. [30] as well. However, with regard to stability, ethylene - air mixtures behaved differently from hydrogen - air mixtures for same initial conditions. For equivalence ratios up to 2 and for similar degrees of overdrive, the fuel rich hydrogen - air mixture exhibited only short period instability.

Bykovskii et al. [31] formulated a mathematical model for a two-dimensional unsteady spin detonation in a stoichiometric hydrogen - oxygen mixture. A two dimensional transverse detonation wave (TDW) structure was calculated. They observed that a periodic rotating transverse detonation propagated if the combustor length was greater than one and a half TDW size. A minimum combustor diameter as a function of stagnation pressure of the injectors and combustor length was also determined. The twodimensional TDW structure obtained in the mixture was in qualitative agreement with those obtained from previous experiments and from numerical simulations for a propane oxygen mixture.

Baklanov et al. [32] modelled a valveless fuel feed system for a low thrust pulse detonation Engine. Three different configurations of combustion chamber were studied in PDE operating on a stoichiometric Hydrogen - air mixture. The aim was to study the influence of chamber configurations on detonation parameters. Experiments were performed in a cylindrical shock tube $1.6 \mathrm{~m}$ long and valveless fuel and oxidant feed was used to fill a cylindrical combustion chamber $0.585 \mathrm{~m}$ long. In the experiments conducted, annular ring obstacles were placed in the combustion chamber with various blockage ratios and they were seen to influence the DDT length. 
Ken Matsuoka et al. [33] proposed a valve system for PDEs that uses an inflowdriven piston-interrupting valve. The valve produces intermittent flow by using gas enthalpy eliminating the need for any power source. The inflow driven valve is a piston interrupting valve, a kind of reciprocal valve in which inflow interrupts the piston. In the experiments conducted, three piston IDVs were used with no energy unit in the valve system. Their study is based on the work by Golovastov et al. [32] who proposed a PDE valve concept where the reciprocating motion of piston is achieved by spring force and enthalpy generation of a detonation wave. The cylinder has a supply and exhaust port, through which the propellant flows into the combustor intermittently. This concept was evaluated by Matsuoka's group by performing thrust measurements and evaluating stable operation.

One of the recent literatures on RDEs is by Liu et al. [34]. They present results from both experimental and three-dimensional numerical studies on hydrogen - air continuous rotating detonation. A single step irreversible Arrhenius kinetics is used for the hydrogen - air mixture. Grid dependence was performed by comparing different one dimensional detonation properties at various grid scales, and a grid resolution of $0.5 \mathrm{~mm}$ was chosen for the study. Detonation was realized for about $300 \mathrm{~ms}$ with propagation frequency between 5.35 and $5.85 \mathrm{kHz}$ in the experiment and between 6.26 and $6.27 \mathrm{kHz}$ in simulations.

The current study incorporates elements like grid sizing and chemical reaction mechanism from the works listed above. A great amount of research has gone into identifying the ideal grid size for detonation problems, the right combustion mechanism for a hydrogen - oxygen mixture, methods to initiate detonation numerically, and determining initial and boundary conditions. Although the dynamics of detonation waves 
have been studied extensively using various commercial CFD softwares, works on the concept of hydrodynamic/fluidic valves in a detonation engine are limited. This work therefore makes use of the knowledge gathered from literature survey to set up the physics in a detonation tube and explores the dynamics of the wave to have a good understanding of the behavior of propellants inside a fluidic valve. The major challenge encountered in this simulation was identifying the right initial and boundary conditions. A lot of experimentation has been done with this regard, but only the final case has been discussed in the following chapter. 
Chapter 3

Methodology

Numerical modelling has been performed using the commercial computational fluid dynamics software tool ANSYS Fluent 17.0. Three steps involved in any numerical modelling / simulation are Preprocessing, Solver and Post processing.

\subsection{Preprocessing}

Preprocessing is the stage where a geometry or physical model is created and a mesh is generated. ANSYS Workbench has an in-built software for creating models called Design Modeler and a meshing software to generate mesh for the associated geometry. One can also create geometries using external CAD packages like AutoCad, CATIA, Solidworks, etc. and use meshing software like Gambit or Pointwise. These files can then be loaded into ANSYS Solver for solution. For this project, the geometry software built in ANSYS has been used as the model is simple and basic.

\subsubsection{Geometry}

The 2D geometry used for the current simulation is based on Figures 1.5 and 1.6 from Chapter 1. The head end of the tube is closed and the other end open. The detonation tube is $3.05 \mathrm{~m}$ long, with a diameter of $0.0508 \mathrm{~m}$ and the $\mathrm{FV}$ placed at a distance of $2.199 \mathrm{~m}$ from the head end of the tube. Since the geometry (Fig 1.5) is symmetric along the horizontal centerline, only one half of the problem is modelled (height of the detonation tube now becomes $0.0254 \mathrm{~m}$ ). The geometry is created in Design Modeler which is the geometry software in WorkBench. The original dimensions of the detonation tube and the fluidic valve are retained as the results from this simulation have to be verified with the results from the experiment. Although dimensions do not affect gas dynamics properties when the case is initialized as an inviscid model, physical 
parameters such as valve time; change with change in dimension. Figure 3.1 is the geometry used and it can be seen that a rectangular chamber has been added at the right end of the tube. This is explained in Section 3.2.2. Although the physical tube geometry is three dimensional, it has been observed that there is very little flow variation in an RDE in the radial direction when compared to its axial and azimuthal directions since the radial dimension is usually small compared to the other two dimensions [11]. This allows the RDE to be unrolled into two dimensions and thus the $2 \mathrm{D}$ geometry used in the simulation relates to the $3 \mathrm{D}$ physical tube geometry.

Figure 3.1: 2D geometry used in the simulation

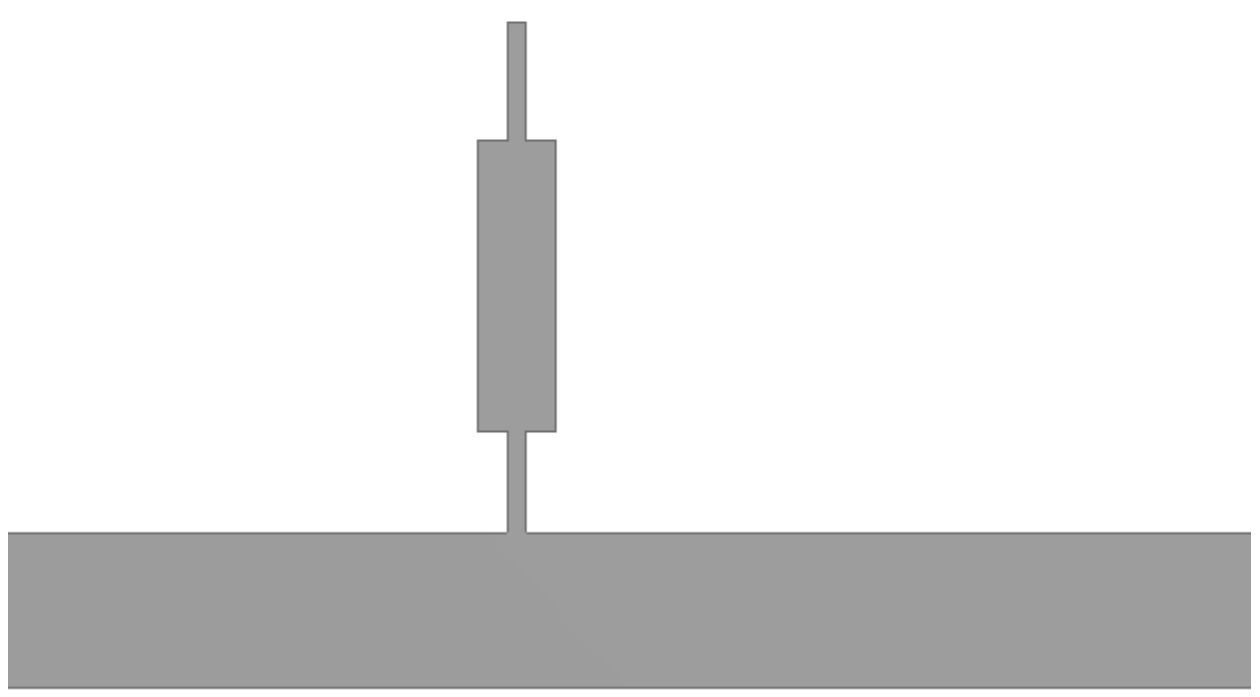

Figure 3.2: Enlarged view of the geometry to show fluidic valve 


\subsubsection{Mesh}

Meshing has been performed with the meshing software Gambit. The geometry is simple and is made of basic shapes such as rectangles making it ideal for structured mesh. Since the tube is $3.05 \mathrm{~m}$ long, it is not necessary to have a uniform fine mesh throughout the geometry. Therefore, non-uniform grids have been used by identifying regions that do not contribute to or change the wave dynamics in any manner and using coarser mesh in those regions. This helps reduce computation time significantly.

The coarsest grid chosen was $3 \times 3 \mathrm{~mm}$ and the finest grid, $0.2 \times 0.2 \mathrm{~mm}$ after performing grid dependent study and referring to relevant papers. The portion of the tube connecting the coarsest and finest regions had unstructured mesh. The distribution of mesh and its details can be found in Figure 3.3 and Table 3.1 respectively. The mesh is finer near and inside the valve because it is of utmost importance for this study to capture the nature and behavior of propellants and combustion products inside the valve. The finer the mesh, the better the flow properties are captured. For the mesh dependent study, grid sizes of $0.05 \mathrm{~mm}, 0.1 \mathrm{~mm}, 0.2 \mathrm{~mm}, 0.3 \mathrm{~mm}, 0.5 \mathrm{~mm}$ and $3 \mathrm{~mm}$ were considered for a simple rectangular geometry. Each of these were run in the manner mentioned in Section 3.1.3. A number of vertical planes were chosen in the rectangular tube and detonation parameters such as CJ Pressure, temperature, detonation velocity etc. were compared at that location for each of the grid sizes. The properties are said to be grid dependent if the values change with grid size. The results from the grid dependent study are presented in Table 3.2, and Table 3.3 shows the deviation of detonation parameters from CEA values for the grid sizes chosen. 

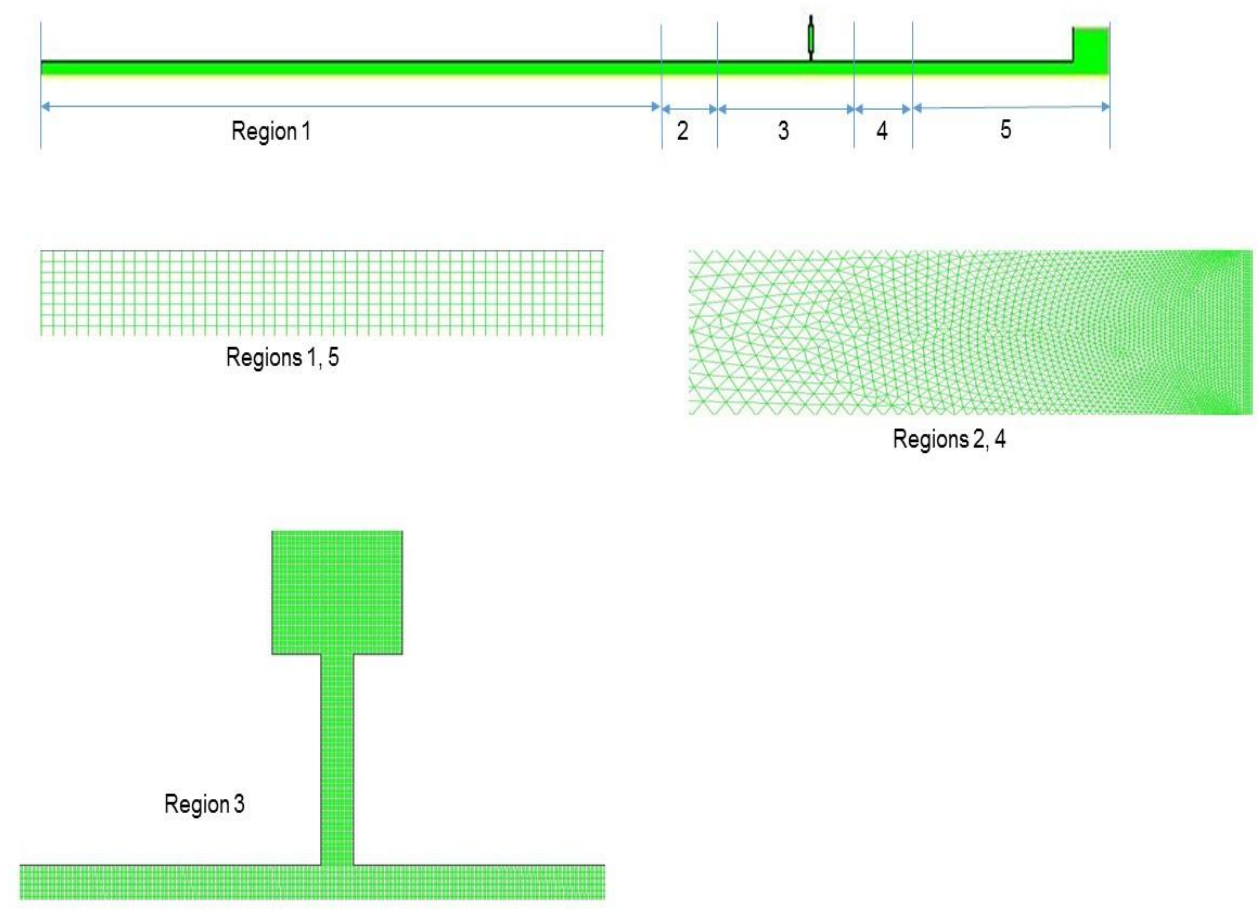

Figure 3.3: Mesh

Table 3.1 Details of Mesh

\begin{tabular}{|l|l|l|l|l|}
\hline Region & Grid Size & Element type & No. of nodes & No. of elements \\
\hline 1 & $3 \times 3 \mathrm{~mm}$ & Structured, Quadrilateral & 6237 & 5536 \\
\hline 2 & Non uniform & Unstructured, Triangular & 2742 & 5279 \\
\hline 3 & $0.2 \times 0.2 \mathrm{~mm}$ & Structured, Quadrilateral & 44548 & 43532 \\
\hline 4 & Non uniform & Unstructured, Triangular & 2753 & 5301 \\
\hline 5 & $3 \times 3 \mathrm{~mm}$ & Structured, Quadrilateral & 2885 & 2604 \\
\hline Total & Mixed & Mixed & 59165 & 62252 \\
\hline
\end{tabular}


Table 3.2 Grid Dependent Study

\begin{tabular}{|c|c|c|c|c|}
\hline $\begin{array}{c}\text { Grid Size } \\
(\mathrm{mm})\end{array}$ & $\begin{array}{c}\text { Location } \\
(\mathrm{m})\end{array}$ & $\begin{array}{c}\text { Pressure } \\
(\mathrm{atm})\end{array}$ & $\begin{array}{c}\text { Temperature } \\
(\mathrm{K})\end{array}$ & $\begin{array}{c}\text { Density } \\
\left(\mathrm{kg} / \mathrm{m}^{3}\right)\end{array}$ \\
\hline 0.05 & 0.01 & 19 & 3700 & 0.92 \\
\hline 0.1 & 0.05 & 19 & 3700 & 0.91 \\
\hline & 0.01 & 19 & 3680 & 0.909 \\
\hline 0.2 & 0.05 & 19 & 3680 & 0.9 \\
\hline & 0.01 & 19 & 3680 & 0.96 \\
\hline 0.3 & 0.05 & 19 & 3690 & 0.9 \\
\hline & 0.01 & 17 & 3640 & 0.82 \\
\hline 0.5 & 0.01 & 18 & 3640 & 0.822 \\
\hline & 0.05 & 18 & 3640 & 0.82 \\
\hline 3 & 0.01 & 16.6 & 3650 & 0.82 \\
\hline & 0.05 & 16.6 & 3650 & 0.82 \\
\hline NASA CEA Values & 18.657 & 3675.9 & 0.9 \\
\hline
\end{tabular}

Therefore, the $0.2 \mathrm{~mm}$ grid size chosen for the study can be justified as it captures the detonation parameters well.

Table 3.3 Deviation of detonation parameters from CEA values

\begin{tabular}{|c|c|c|c|}
\hline \multirow{2}{*}{$\begin{array}{c}\text { Grid Size } \\
(\mathrm{mm})\end{array}$} & \multicolumn{3}{|c|}{ Deviation from CEA values } \\
\cline { 2 - 4 } & Pressure & Temperature & Density \\
\hline 0.2 & $1.8 \%$ & $0.2 \%$ & $3.3 \%$ \\
\hline 3 & $-11.02 \%$ & $-0.7 \%$ & $-8.88 \%$ \\
\hline
\end{tabular}




\title{
3.2 Solver
}

Solver is the stage where the physics of the problem is defined and the solution is initialized.

A 2D, double precision solver is used for the present model. Although single precision solvers provide sufficient accuracy, it is advised to use double precision solvers where the geometry involves very long, thin pipes so that all nodes are represented [35]. Next, the case is setup by specifying the models, materials and boundary conditions. In the 'general' tab of Fluent setup, the following options are checked -

\author{
Solver type: Density Based \\ Velocity formulation: Absolute \\ Time: Transient \\ 2D space: Planar
}

The two traditional flow solvers available in Fluent are Pressure based solver and Density based solver. A Density based solver has been chosen for the simulation of detonation as these solvers are intended for high speed compressible flows. Both solvers solve the governing equations for conservation of mass, momentum, and energy and other scalars and employ a finite-volume discretization process, but they differ in their approach of linearizing and solving the discretized equations [35]. Density based solvers solve continuity, momentum, energy and species transport equations simultaneously. In order to obtain a converged solution, several iterations have to be performed as the governing equations are coupled and non-linear [35]. The schematic below represents the sequence of iteration in a density based solver. This is repeated until the solution converges. 


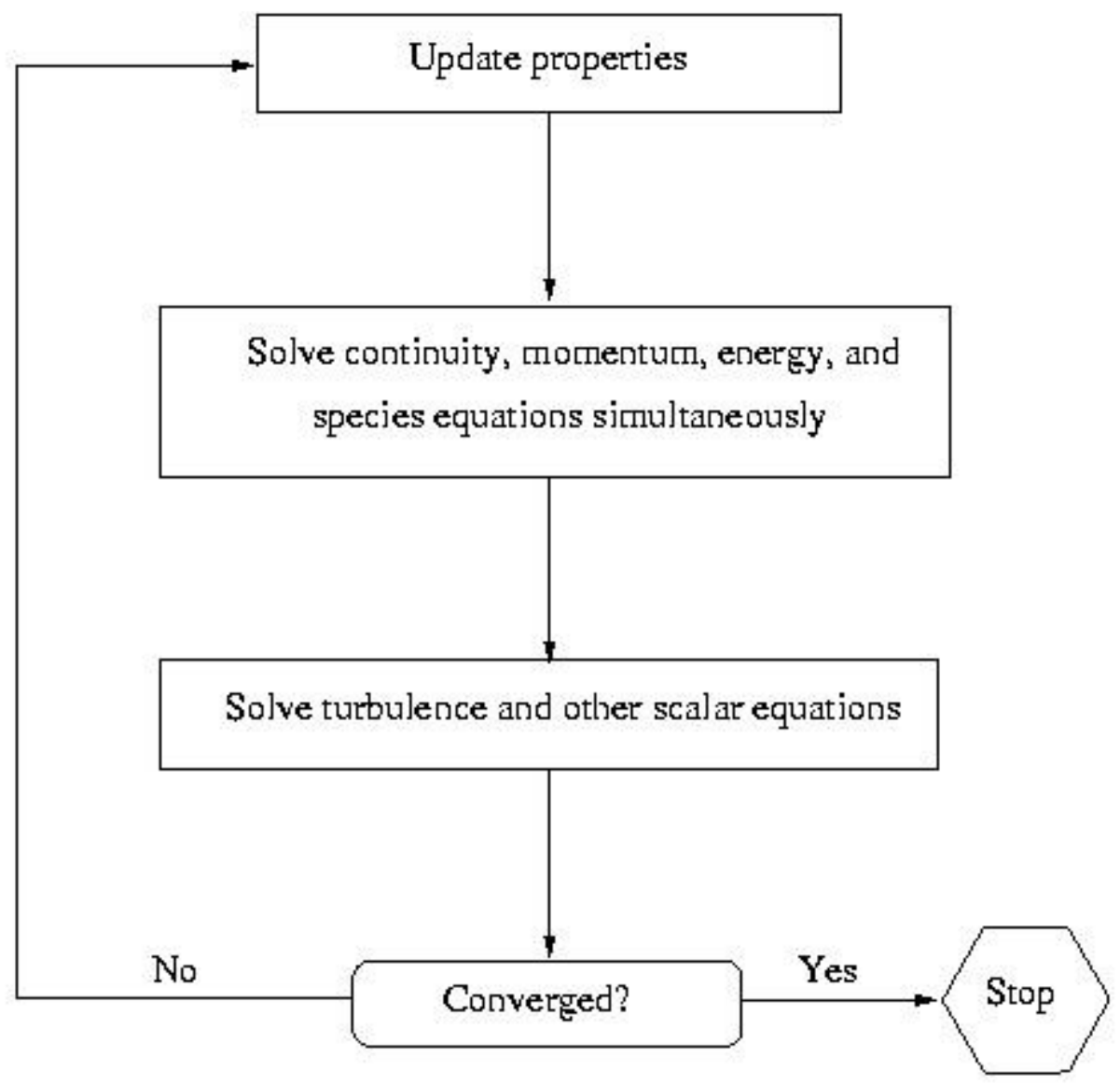

Figure 3.4: Sequence of Iteration [35]

Density based solvers offer two ways in which the coupled system of equations can be solved - implicit and explicit formulation. Explicit formulations are much simpler in the sense that they do not require current values at every iteration to solve the equations [36]. In the explicit method, all the variables are solved one cell at a time, while in the implicit formulation, variables in all cells are solved simultaneously. Therefore, the implicit method takes more computation and more memory although the solution converges faster. Explicit formulation is used in cases where the time step size is very small. In detonation, the time scale of chemical reactions is so small that it is in the order of 
nanoseconds. For the detonation studied in this project, the time step size is $10^{-9} \mathrm{~s}$ and therefore, the explicit formulation has been chosen. This corresponds to a Damkohler number (ratio of convective time scale to reaction time scale) of unity and a CFL number around 0.1. Low values of CFL number help the solution converge faster. Literature on numerical simulation of detonations show that these are indeed the standard numbers. A multi-stage Runge-Kutta time integration method has been used, which is traditional in density based solvers using explicit formulation. An AUSM (Advection Upstream Splitting Method) scheme is chosen for computing flux vectors because of its simplicity and accuracy in capturing shock discontinuities [37]. For spacial discretization, a second order upwind scheme and Least Squares cell based gradient have been used.

Energy model is turned on and the case is run as an inviscid flow. These options can be specified under 'Models' in the setup. Although detonation is a phenomenon involving viscosity and turbulence, initializing it with an inviscid model still captures most of the detonation properties [17]. The model was chosen for the sake of simplicity, although it must be noted that it doesn't affect the results to a great extent.

Fluent solves reactive Euler equations for inviscid flows. The governing equations are as follows.

$$
\begin{aligned}
& (\rho)_{t}+(\rho u)_{x}+(\rho v)_{y}=0 \\
& (\rho u)_{t}+\left(\rho u^{2}+P\right)_{x}+(\rho u v)_{y}=0 ;(\rho v)_{t}+(\rho u v)_{x}+\left(\rho v^{2}+P\right)_{y}=0 \\
& (\rho E)_{t}+(\rho u E+u P)_{x}+(\rho v E+v P)_{y}=0 \\
& \left(\rho Y_{n}\right)_{t}+\left(\rho u Y_{n}\right)_{x}+\left(\rho v Y_{n}\right)_{y}=R_{n}
\end{aligned}
$$

where the subscripts represent partial derivatives. 
The equation of state is used to determine pressure. For density calculations in compressible flows, ideal gas law is used:

$\rho=\left(P_{o p}+P\right) /\left(R T / M_{w}\right)$

The specific heat at constant pressure is calculated using mixing law and the specific heat for the individual species is evaluated by a two-temperature range piecewise polynomial $-300 \mathrm{~K}$ to $1000 \mathrm{~K}$ and $1000 \mathrm{~K}$ to $5000 \mathrm{~K}$.

A finite rate chemistry model is used for the combustion process and it is used to determine reaction rates. The forward reaction rate constant for a reaction $r$ is given by the Arrhenius expression,

$k_{f, r}=A_{r} T^{\beta} e^{-E r / R T}$

The backward rate constant for a reversible reaction is given by

$\mathrm{k}_{\mathrm{b}, \mathrm{r}}=\mathrm{k}_{\mathrm{f}, \mathrm{r}} / \mathrm{K}_{\mathrm{r}}$

A detailed, 19-step finite rate reaction is used to model an eight species hydrogen - oxygen detonation. This combustion mechanism is based on the work of Jachimowski, with slight modifications by Wilson and MacCormack which involves 33 reactions and 13 reaction species as shown below [38 -39]. 
Table 3.4 Hydrogen - Air Combustion Mechanism [38]

\begin{tabular}{|c|c|c|c|c|}
\hline$j$ & Reaction $^{c} j$ & $A_{j}$ & $n_{j}$ & $E_{j}$ \\
\hline$\left(1^{\prime}\right)$ & $\mathrm{H}_{2}+\mathrm{O}_{2} \rightleftharpoons \mathrm{HO}_{2}+\mathrm{H}$ & $1.00 \times 10^{14}$ & 0 & 56,000 \\
\hline (2) & $\mathrm{H}+\mathrm{O}_{2} \rightleftharpoons \mathrm{OH}+\mathrm{O}$ & $2.60 \times 10^{14}$ & 0 & 16,800 \\
\hline (3) & $\mathrm{O}+\mathrm{H}_{2} \rightleftharpoons \mathrm{OH}+\mathrm{H}$ & $1.80 \times 10^{10}$ & 1.00 & 8,900 \\
\hline (4) & $\mathrm{OH}+\mathrm{H}_{2} \rightleftharpoons \mathrm{H}_{2} \mathrm{O}+\mathrm{H}$ & $2.20 \times 10^{13}$ & 0 & 5,150 \\
\hline (5) & $\mathrm{OH}+\mathrm{OH} \rightleftharpoons \mathrm{H}_{2} \mathrm{O}+\mathrm{O}$ & $6.30 \times 10^{12}$ & 0 & 1,090 \\
\hline (6) & $\mathrm{H}+\mathrm{OH}+M \rightleftharpoons \mathrm{H}_{2} \mathrm{O}+M$ & $2.20 \times 10^{22}$ & -2.00 & 0 \\
\hline (7) & $\mathrm{H}+\mathrm{H}+M \rightleftharpoons \mathrm{H}_{2}+M$ & $6.40 \times 10^{17}$ & -1.00 & 0 \\
\hline (8) & $\mathrm{H}+\mathrm{O}+M \rightleftharpoons \mathrm{OH}+M$ & $6.00 \times 10^{16}$ & -0.6 & 0 \\
\hline (9) & $\mathrm{H}+\mathrm{O}_{2}+M \rightleftharpoons \mathrm{HO}_{2}+M$ & $2.10 \times 10^{15}$ & 0 & $-1,000$ \\
\hline (10) & omitted & & & \\
\hline (11) & $\mathrm{HO}_{2}+\mathrm{H} \rightleftharpoons \mathrm{OH}+\mathrm{OH}$ & $1.40 \times 10^{14}$ & 0 & 1,080 \\
\hline (12) & $\mathrm{HO}_{2}+\mathrm{H} \rightleftharpoons \mathrm{H}_{2} \mathrm{O}+\mathrm{O}$ & $1.00 \times 10^{13}$ & 0 & 1.080 \\
\hline (13) & $\mathrm{HO}_{2}+\mathrm{O} \rightleftharpoons \mathrm{O}_{2}+\mathrm{OH}$ & $1.50 \times 10^{13}$ & 0 & 950 \\
\hline (14) & $\mathrm{HO}_{2}+\mathrm{OH} \rightleftharpoons \mathrm{H}_{2} \mathrm{O}+\mathrm{O}_{2}$ & $8.00 \times 10^{12}$ & 0 & 0 \\
\hline (15) & $\mathrm{HO}_{2}+\mathrm{HO}_{2} \rightleftharpoons \mathrm{H}_{2} \mathrm{O}_{2}+\mathrm{O}_{2}$ & $2.00 \times 10^{12}$ & 0 & 0 \\
\hline (16) & $\mathrm{H}+\mathrm{H}_{2} \mathrm{O}_{2} \rightleftharpoons \mathrm{H}_{2}+\mathrm{HO}_{2}$ & $1.40 \times 10^{12}$ & 0 & 3,600 \\
\hline (17) & $\mathrm{O}+\mathrm{H}_{2} \mathrm{O}_{2} \rightleftharpoons \mathrm{OH}+\mathrm{HO}_{2}$ & $1.40 \times 10^{13}$ & 0 & 6,400 \\
\hline (18) & $\mathrm{OH}+\mathrm{H}_{2} \mathrm{O}_{2} \rightleftharpoons \mathrm{H}_{2} \mathrm{O}+\mathrm{HO}_{2}$ & $6.10 \times 10^{12}$ & 0 & 1,430 \\
\hline (19) & $M+\mathrm{H}_{2} \mathrm{O}_{2} \rightleftharpoons \mathrm{OH}+\mathrm{OH}+M$ & $1.20 \times 10^{17}$ & 0 & 45,500 \\
\hline (20) & $\mathrm{O}+\mathrm{O}+M=\mathrm{O}_{2}+M$ & $6.00 \times 10^{13}$ & 0 & $-1,800$ \\
\hline (21) & $\mathrm{N}+\mathrm{N}+M \rightleftharpoons \mathrm{N}_{2}+M$ & $2.80 \times 10^{17}$ & -0.75 & 0 \\
\hline (22) & $\mathrm{N}+\mathrm{O}_{2} \rightleftharpoons \mathrm{NO}+\mathrm{O}$ & $6.40 \times 10^{19}$ & 1.00 & 6,300 \\
\hline (23) & $\mathrm{N}+\mathrm{NO} \rightleftharpoons \mathrm{N}_{2}+\mathrm{O}$ & $1.60 \times 10^{13}$ & 0 & 0 \\
\hline (24) & $\mathrm{N}+\mathrm{OH} \rightleftharpoons \mathrm{NO}+\mathrm{H}$ & $6.30 \times 10^{11}$ & 0.50 & 0 \\
\hline (25) & $\mathrm{H}+\mathrm{NO}+M \rightleftharpoons \mathrm{HNO}+M$ & $5.40 \times 10^{15}$ & 0 & -600 \\
\hline (26) & $\mathrm{H}+\mathrm{HNO} \rightleftharpoons \mathrm{NO}+\mathrm{H}_{2}$ & $4.80 \times 10^{12}$ & 0 & 0 \\
\hline (27) & $\mathrm{O}+\mathrm{HNO} \rightleftharpoons \mathrm{NO}+\mathrm{OH}$ & $5.00 \times 10^{11}$ & 0.50 & 0 \\
\hline (28) & $\mathrm{OH}+\mathrm{HNO} \rightleftharpoons \mathrm{NO}+\mathrm{H}_{2} \mathrm{O}$ & $3.60 \times 10^{13}$ & 0 & 0 \\
\hline (29) & $\mathrm{HO}_{2}+\mathrm{HNO} \rightleftharpoons \mathrm{NO}+\mathrm{H}_{2} \mathrm{O}_{2}$ & $2.00 \times 10^{12}$ & 0 & 0 \\
\hline (30) & $\mathrm{HO}_{2}+\mathrm{NO} \rightleftharpoons \mathrm{NO}_{2}+\mathrm{OH}$ & $3.40 \times 10^{12}$ & 0 & -260 \\
\hline (31) & $\mathrm{H}+\mathrm{NO}_{2} \rightleftharpoons \mathrm{NO}+\mathrm{OH}$ & $3.50 \times 10^{14}$ & 0 & 1,500 \\
\hline (32) & $\mathrm{O}+\mathrm{NO}_{2} \rightleftharpoons \mathrm{NO}+\mathrm{O}_{2}$ & $1.00 \times 10^{13}$ & 0 & 600 \\
\hline (33) & $M+\mathrm{NO}_{2} \rightleftharpoons \mathrm{NO}+\mathrm{O}+M$ & $1.16 \times 10^{16}$ & 0 & 66,000 \\
\hline
\end{tabular}

'Third body efficiencies relative to $\mathrm{N}_{2}$ :

Reaction (6) $\mathrm{H}_{2} \mathrm{O}=6.0$

Reaction (7) $\mathrm{H}_{2} \mathrm{O}=6.0 ; \mathrm{H}_{2}=2.0$

Reaction (8) $\mathrm{H}_{2} \mathrm{O}=5.0$

Reaction (9) $\mathrm{H}_{2} \mathrm{O}=16.0 ; \mathrm{H}_{2}=2.0$

Reaction (19) $\mathrm{H}_{2} \mathrm{O}=15.0$ 
Only the first 19 reactions from the above mechanism are chosen for the Hydrogen Oxygen detonation studied in this project as the 33 step reaction mechanism is proposed for Hydrogen - air combustion. Therefore, reactions involving Nitrogen are eliminated.

\subsubsection{Chemical Kinetics}

Hydrogen - oxygen detonation is a detailed and complex chemical process. Such a combustion process involves three steps namely Initiation, Propagation and Termination. A lot of mechanisms have been proposed for hydrogen - air detonations, ranging from the basic global mechanism involving 1 or 2-step reactions, the reduced elementary reaction consisting of 7 or 8 -step reactions, to detailed mechanisms involving $21,32,33,38$-step reactions and so on. For the hydrogen - oxygen detonation studied in this research, a single step global mechanism was used initially, but the results were inaccurate and inadequate as most works suggest. Therefore, the ideal choice was to go for a detailed chemical mechanism. The success or ability of the 19-step reaction to capture the detonation physics $[39,40]$ led to it being the natural choice for the current study. Only the results from the detailed 19-step reaction have been presented in this paper as the results from the 1-step reaction are not sufficient or worth mentioning.

\subsubsection{Boundary Condition}

The head end of the tube is closed and treated as a wall while at the exit, a chamber has been added to emulate ambient conditions. It is initialized with a pressure farfield boundary condition with $P=1 \mathrm{~atm}$ and $T=300 \mathrm{~K}$. The fluidic valve inlet is specified by a pressure inlet condition with oxygen flowing in. The total pressure at the

inlet is $4 \mathrm{~atm}$ and a static pressure slightly lower than the total pressure (3.8 atm) is specified to ensure flow into the cavity. All other edges are treated as walls except the bottom edge of the tube, which is symmetric. This is because only one half of the tube is 
modelled and it can be seen from experimental setup that a second valve is located radially opposite to the one at the top and injection in both the valves occur simultaneously.

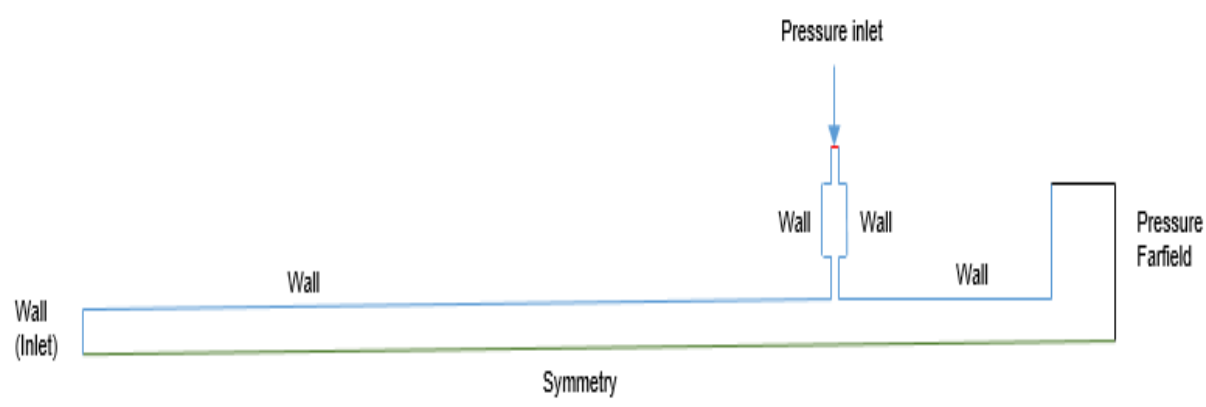

Figure 3.5: Boundary Conditions

\subsubsection{Initial Condition}

Initial conditions of pressure, temperature and mass fractions of reactant species are specified. The detonation tube is filled with a stoichiometric hydrogen - oxygen mixture (mass fraction of $\mathrm{H}_{2}=0.11, \mathrm{O}_{2}=0.89$ ) maintained at 1 atm pressure and $300 \mathrm{~K}$. The fluidic valve is maintained at a constant pressure of $3.8 \mathrm{~atm}, \mathrm{O}_{2}$ mass fraction $=1$ and $300 \mathrm{~K}$.

Detonation is initiated at the head end of tube. In numerical simulations, this is achieved by patching a few cells at the head end of the tube with very high pressure and temperature, usually the conditions at Von Neumann spike (refer Figure 3.6). For hydrogen-oxygen detonations, the cells are patched with $\mathrm{P}=35 \mathrm{~atm}, \mathrm{~T}=3500 \mathrm{~K}$ and species, in the state they are at the end of the 19-step chemical reaction. The value of 
mass fractions of species at the end of a hydrogen-oxygen detonation is predicted by NASA CEA program and is tabulated in Table 3.5.

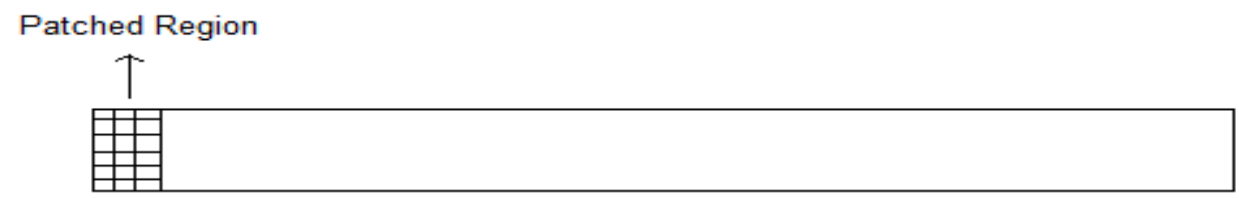

Figure 3.6 Portion of the detonation tube showing patched region

Table 3.5 Species concentration ${ }^{[41]}$

\begin{tabular}{|l|l|}
\hline Species & Mass Fraction \\
\hline $\mathrm{H}$ & 0.00556 \\
\hline $\mathrm{HO}_{2}$ & 0.00042 \\
\hline $\mathrm{H}_{2}$ & 0.02254 \\
\hline $\mathrm{H}_{2} \mathrm{O}$ & 0.66084 \\
\hline $\mathrm{H}_{2} \mathrm{O}_{2}$ & 0.00005 \\
\hline $\mathrm{O}$ & 0.04130 \\
\hline $\mathrm{OH}$ & 0.16593 \\
\hline $\mathrm{O} 2$ & 0.10336 \\
\hline 3.2. & \\
\hline
\end{tabular}

3.2.4 Computational Resource 
The support of the Texas Advanced Computing Center (TACC) for providing the computing resource for this project is sincerely appreciated Running Fluent on a PC takes an incredible amount of time and memory for problems as complicated as detonation. Simulations for this project have been run on Stampede, one of the supercomputers at TACC. The supercomputer is accessed remotely through WinSCP which helps to transfer files securely between a remote computer and a local computer. Preprocessing, physics setup and solution initialization is done on the local computer. The case and data file from Fluent are then transferred to the supercomputer along with an input file. The results or output files are then copied to the local system and loaded into CFD Post for post processing.

\subsection{Post Processing}

Post processing is process of analyzing results from simulations. It has been done in CFD Post, the postprocessor available in Ansys Workbench. The main advantage of CFD Post is that all data files from the simulation can be loaded as a single case. It helps in visualizing data and analyzing results qualitatively and quantitatively. 
Chapter 4

Results and Discussion

In this chapter, the results from the simulation are presented and discussed. Various plots and contours are analyzed to observe detonation properties and pattern of flow. Parameters such as detonation velocity, interruption time, valve time etc. are also determined.

\subsection{Effect of grid resolution on flow variables}

Pressure and temperature are plotted as a function of tube length at instances where the wave crosses the various mesh regions explained in Section 3.1.2.

At $0.078 \mathrm{~ms}$, the wave propagates through Region 1 (coarser grid). The plots below show distribution of properties behind the wave. It can be seen that a peak static pressure of $16.5 \mathrm{~atm}$ and temperature of $3650.46 \mathrm{~K}$ are attained. These values confirm the parameters obtained from the grid study. The end wall conditions also resemble the conditions behind the Taylor expansion wave which verifies the accuracy of the code.

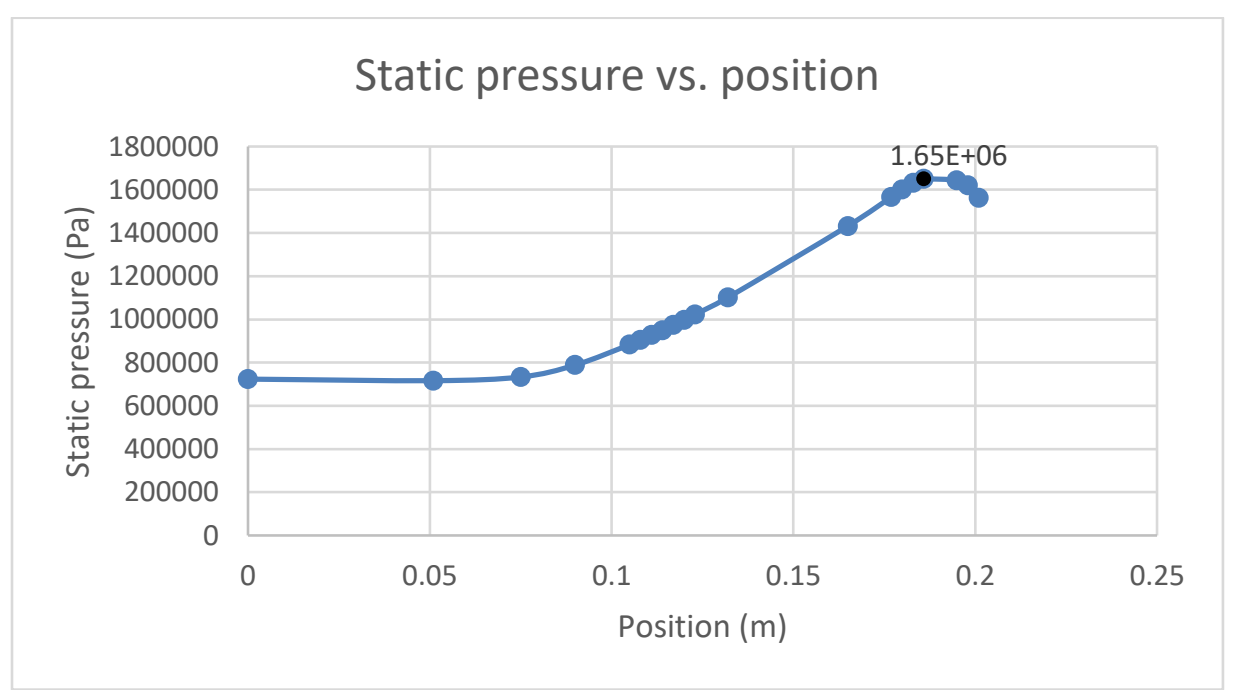

Figure 4.1 Static pressure vs. position at $0.078 \mathrm{~ms}$ 


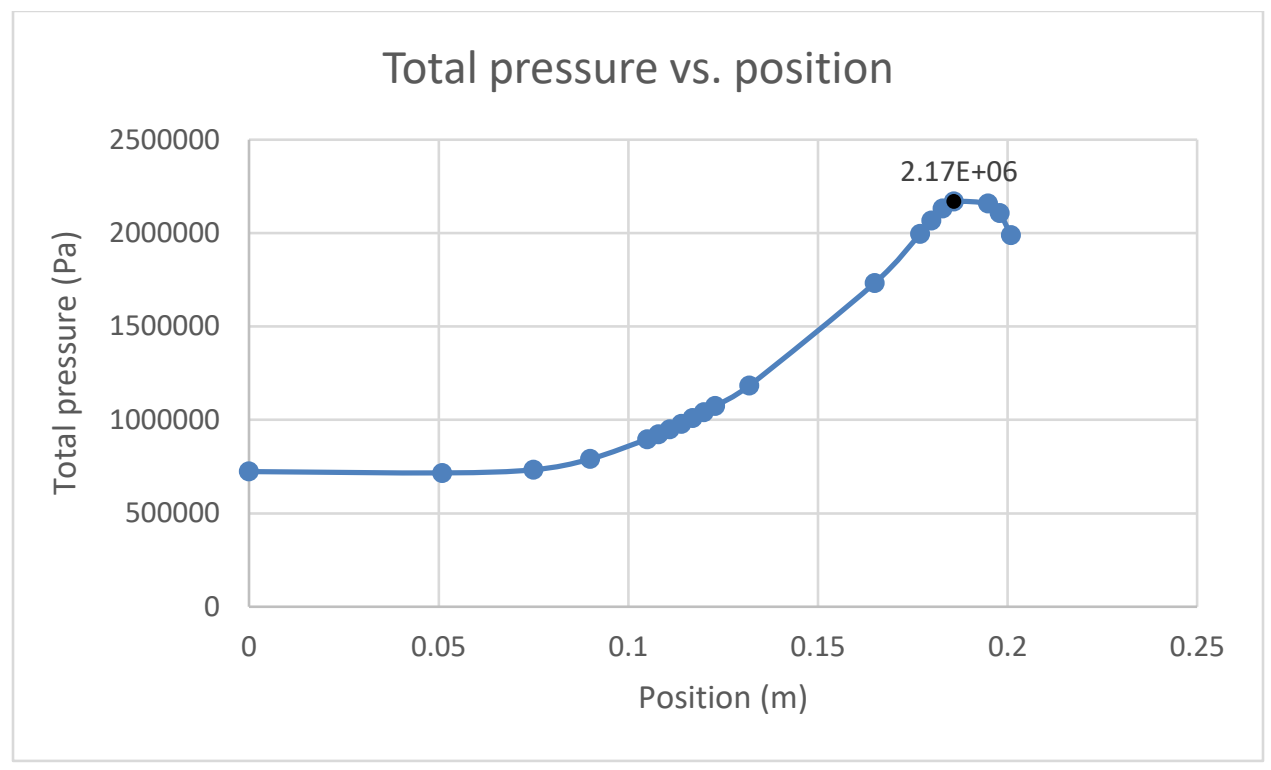

Figure 4.2 Total pressure vs. position at $0.078 \mathrm{~ms}$

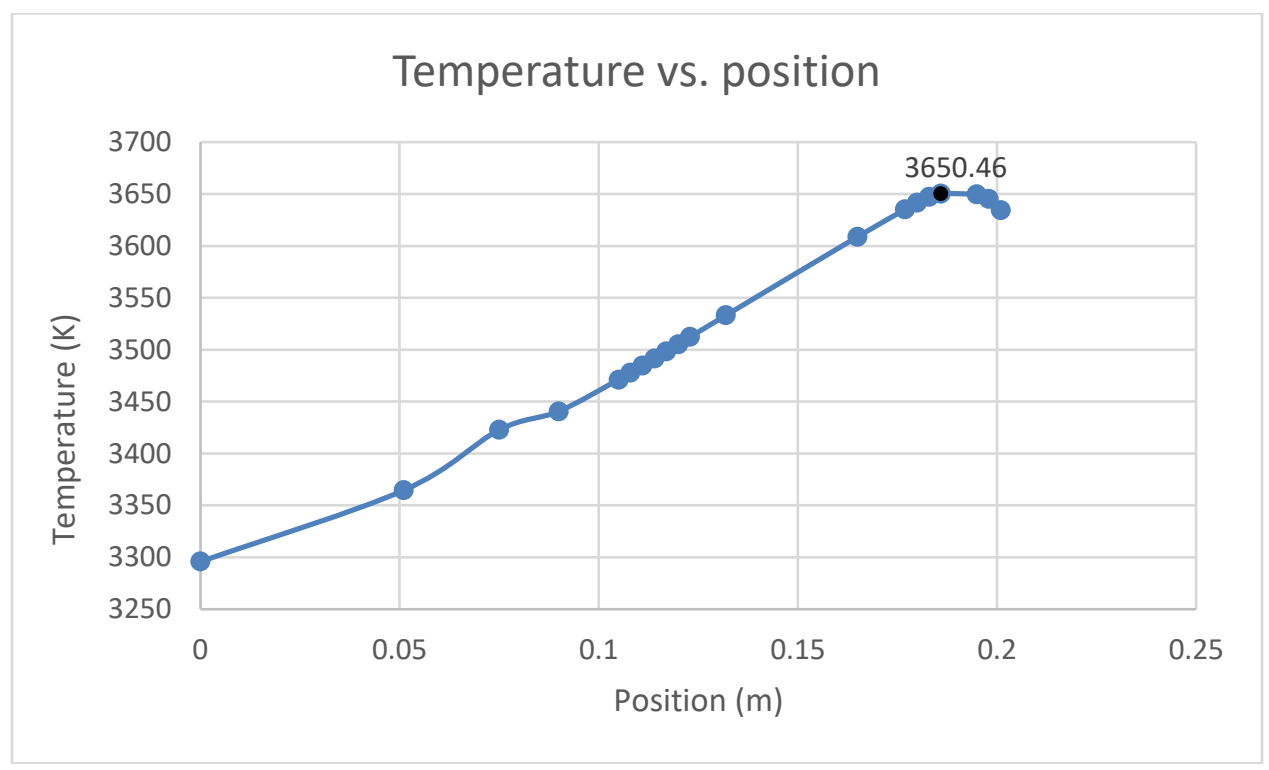

Figure 4.3 Temperature vs. position at $0.078 \mathrm{~ms}$ 
At $0.665 \mathrm{~ms}$, the detonation wave just enters Region 3. Temperature and pressure of the wave front at this instant are denoted by red markers in the plots below. These values match the CJ properties very closely due to fine grid size. Another peak (black markers) is also observed at some distance behind the wave front. This could be due to the variation in grid size as the region ahead of it has unstructured mesh. A more detailed study may be needed to confirm this and to eliminate the possibility of a computational trait, but, such spikes were not observed in the grid study where uniform mesh was used in a simple rectangular geometry..

From the plot of Mach number vs. position, it can be seen that the leading front travels at $M=1$.

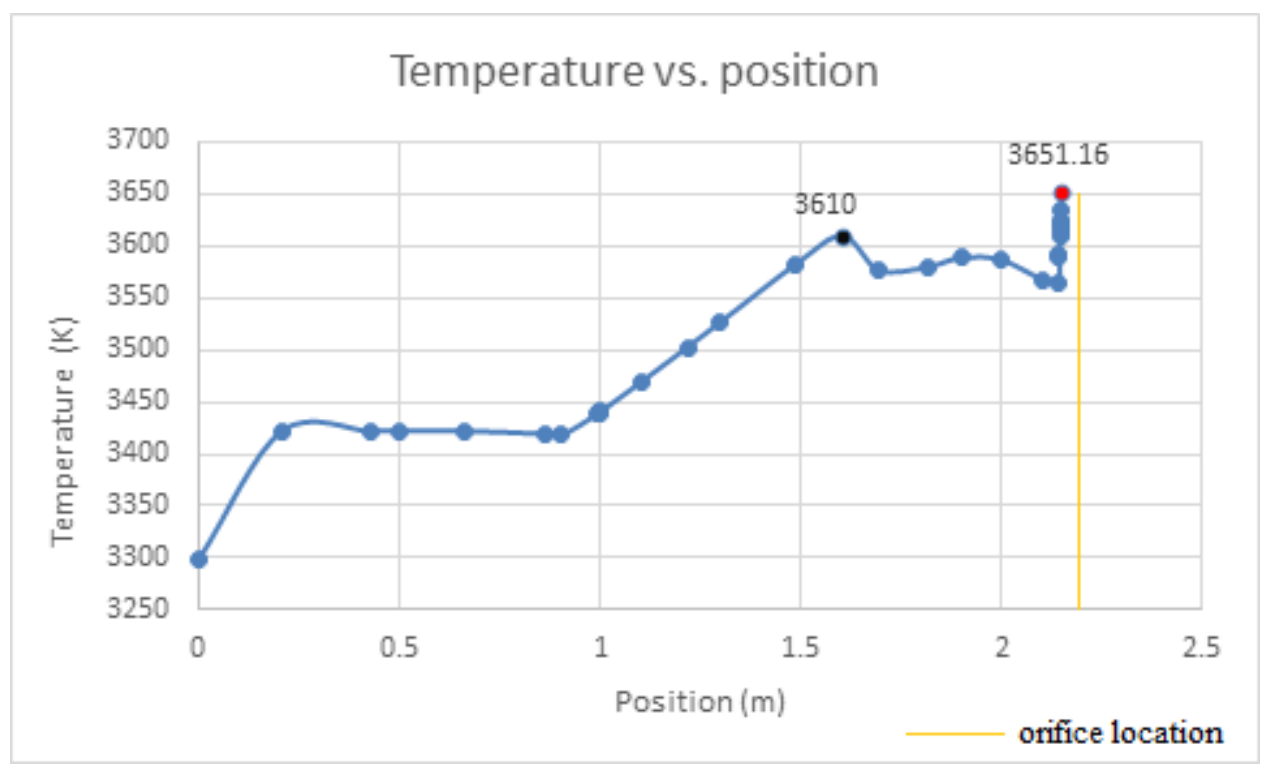

Figure 4.4 Temperature vs. position at $0.665 \mathrm{~ms}$ 


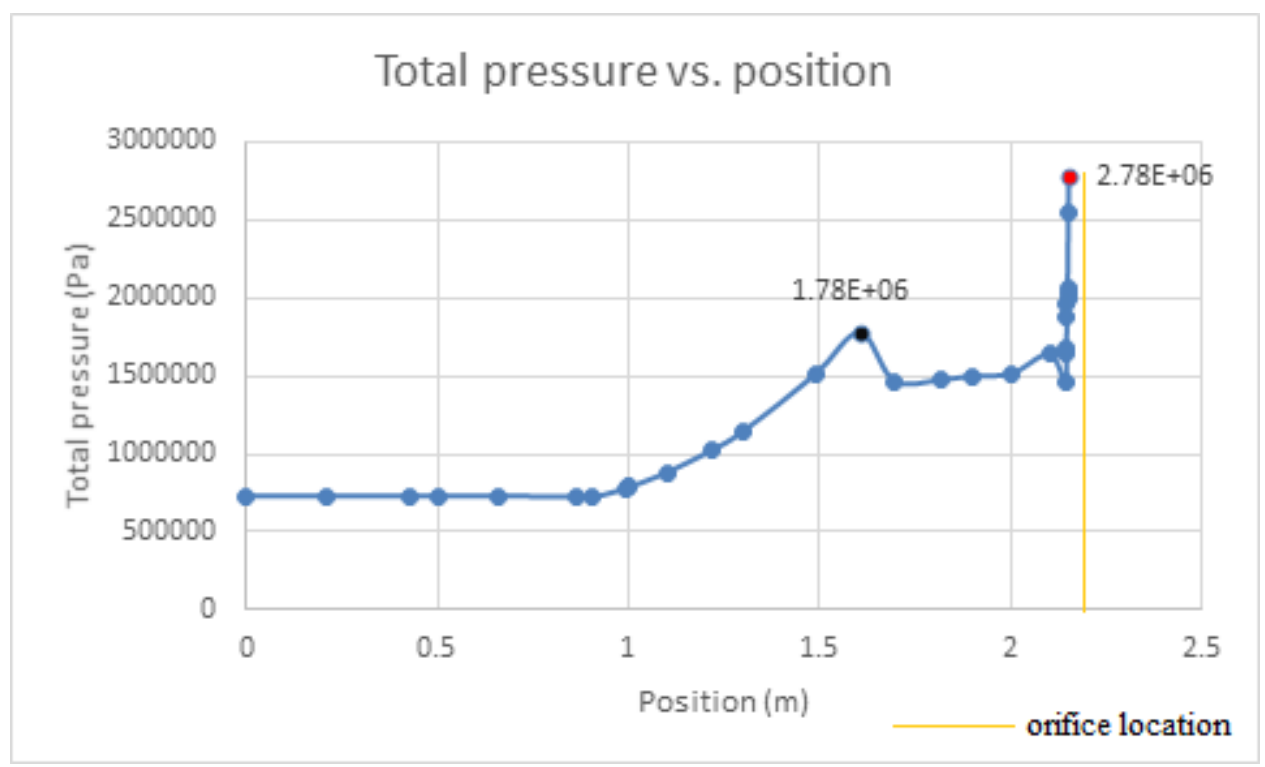

Figure 4.5 Total pressure vs. position at $0.665 \mathrm{~ms}$

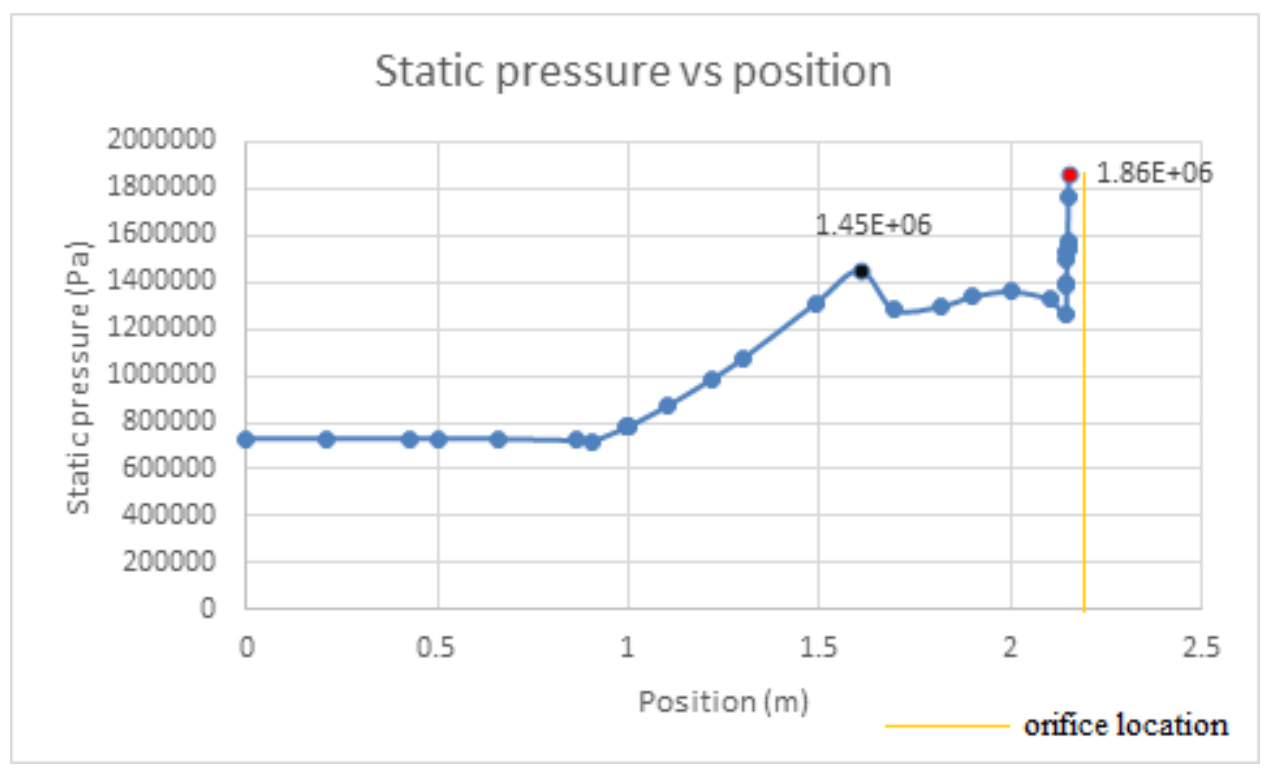

Figure 4.6 Static pressure vs. position at $0.665 \mathrm{~ms}$ 


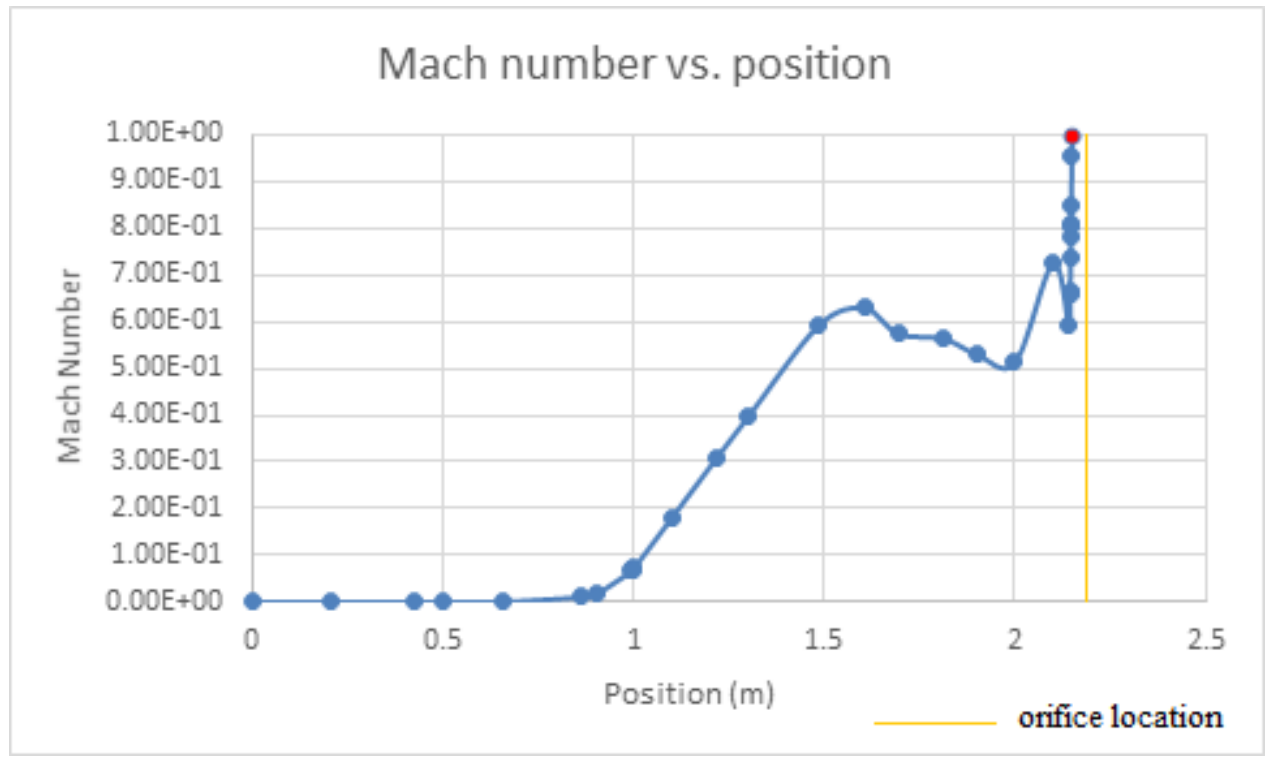

Figure 4.7 Mach number vs. position at $0.665 \mathrm{~ms}$

\subsection{Calculation of detonation velocity}

Detonation velocity is calculated using time of flight method. Two points $\left(\mathrm{x}_{1}, \mathrm{x}_{2}\right.$ : $0.379 \mathrm{~m}, 0.59 \mathrm{~m}$ ) are chosen along the length of the detonation tube and their corresponding times ( $\left.\mathrm{t}_{1}, \mathrm{t}_{2}: 186 \mu \mathrm{s}, 111 \mu \mathrm{s}\right)$ are noted. The detonation velocity is calculated as

$\mathrm{V}=\left(\mathrm{x}_{2}-\mathrm{x}_{1}\right) /\left(\mathrm{t}_{2}-\mathrm{t}_{1}\right)$

$V=2813.33 \mathrm{~m} / \mathrm{s}$, which is very close to the value of CJ velocity obtained from CEA:

$2835.7 \mathrm{~m} / \mathrm{s}$, error being only $0.78 \%$.

It should be noted that any two points which have the same value of pressure at the detonation wave front can be chosen to determine the detonation velocity by using the method above. 


\subsection{Visualization and determination of important flow parameters}

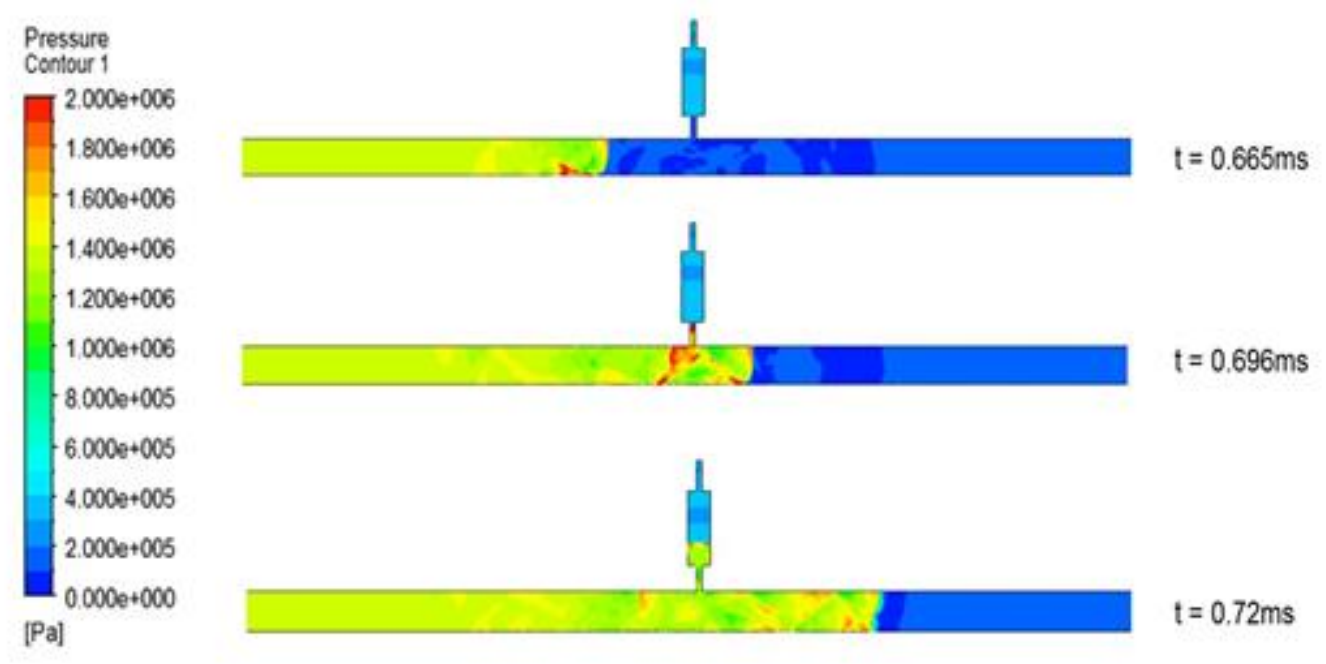

Figure 4.8 Static pressure contours at different instances

The contours above show the propagation of wave at three different times and it can be seen in each of the contours that the pressure at the wave front is very close to the $\mathrm{CJ}$ value. It is also interesting to note that the wave front is not planar as there seems to be a transverse deformation. This is due to a timing issue where oxygen flow should ideally reach the exit of the orifice at the same time the detonation wave reaches the orifice location, but it was not possible to time it so closely even in experiments. Therefore, the deformation of the wave front could be due to the interaction between the oxygen flowing from the valve into the tube and the wave front propagating in the main tube. This can be visualized better with the help of Figure 4.10. 


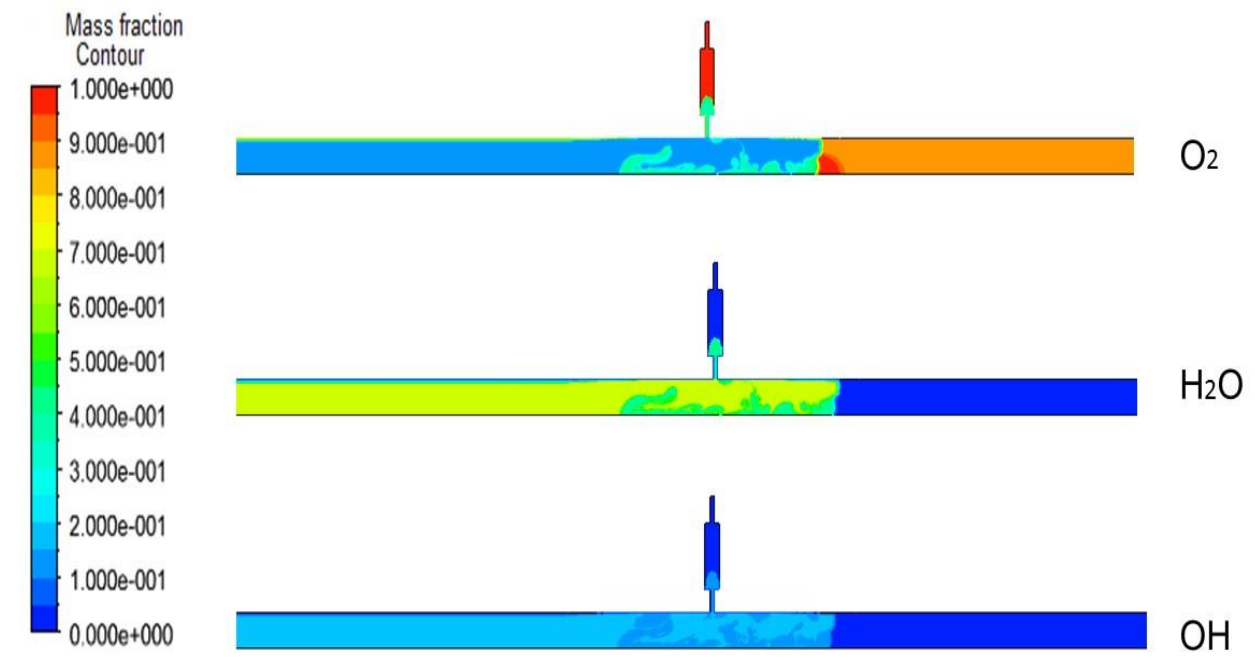

Figure 4.9 Species concentration at $0.72 \mathrm{~ms}$

The contours above show the concentration of $\mathrm{O}_{2}, \mathrm{H}_{2} \mathrm{O}$ and $\mathrm{OH}$ at $0.72 \mathrm{~ms}$. At this instant, the detonation wave has travelled past the fluidic valve and the combustion products have entered the cavity, replacing the oxygen present there. Distribution of other species have not been included as they have negligible mass fractions. 
The contours below represent the flow of oxygen from the valve into main tube. It is seen that oxygen enters the tube before the wave front reaches the orifice.

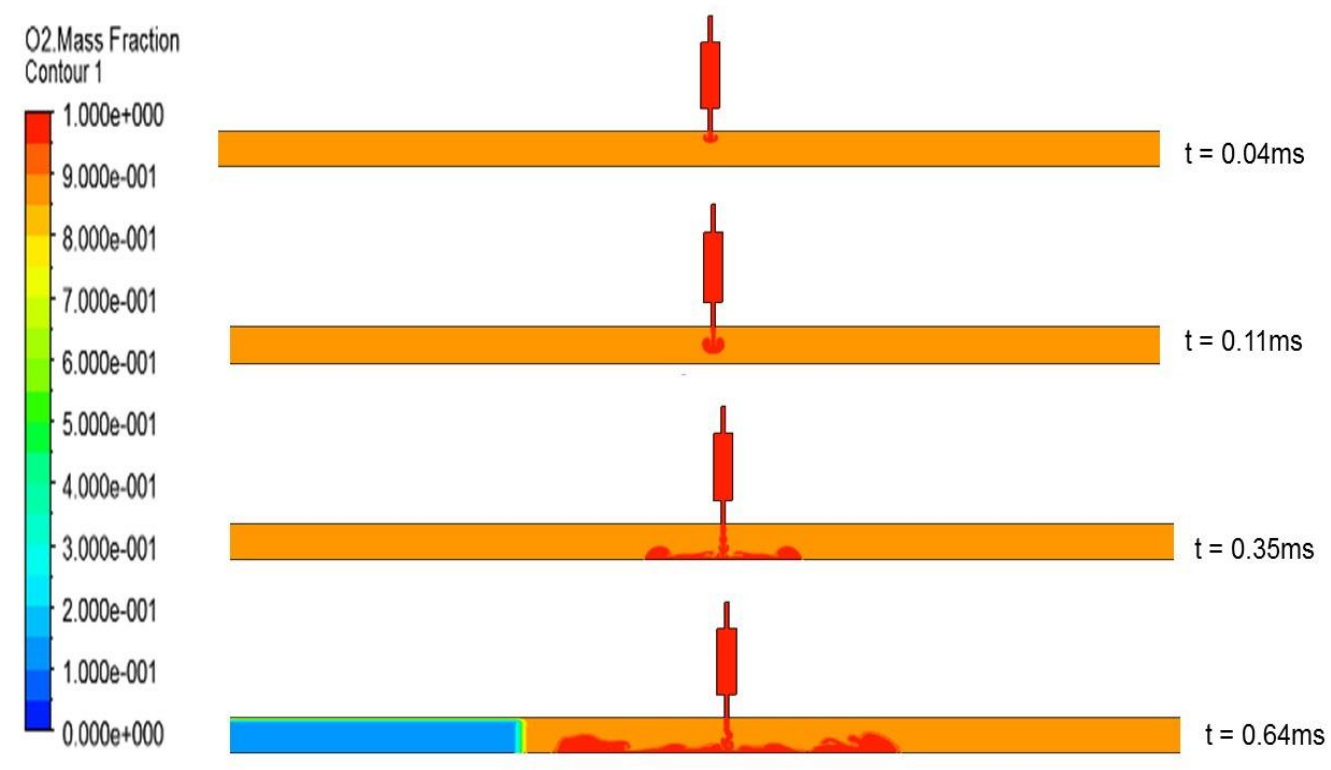

Figure 4.10 Injection of Oxygen into the tube 
Three points are randomly chosen in the plenum cavity as shown in the figure below. Pressure, temperature, density and species contours at these points are plotted to determine the following:

- Time taken for pressure to return to steady state value in the cavity.

- Concentration of species when steady state is reached. This will tell us if steady state flow consists of fresh propellant or detonation products.

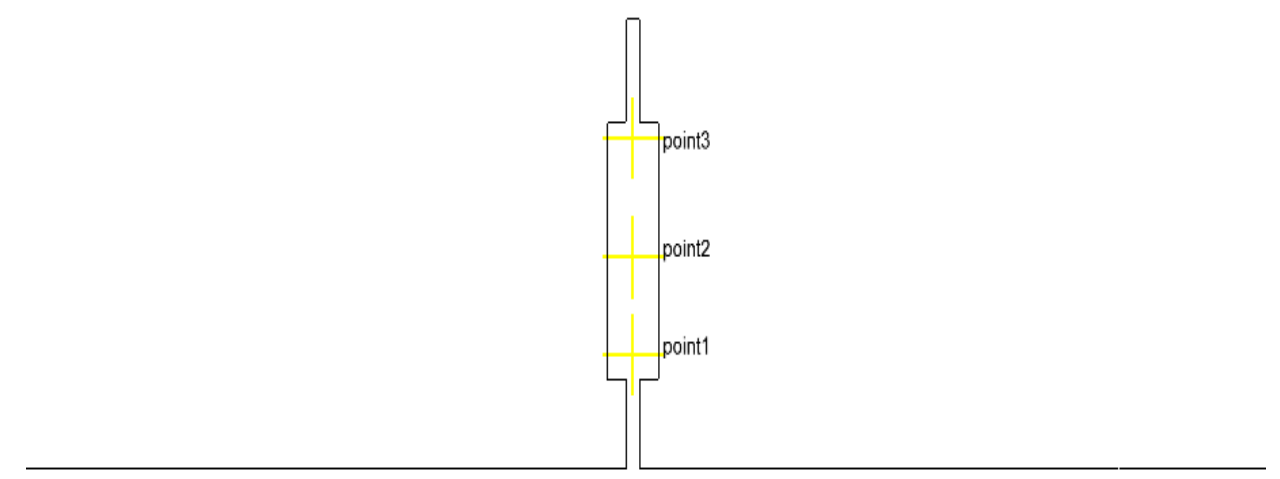

Figure 4.11 Location of points in the plenum cavity

A parameter called the "interruption time" was defined by Braun as the time between the blast wave front entering the plenum cavity and time taken to reach steady state pressure again in the plenum cavity [6]. This can be determined easily with the help of a plot of pressure vs. time. Thus, the interruption time is determined graphically by averaging the values obtained from the pressure plots at points 1,2 and 3 . 
Static Pressure vs. time at Point 1

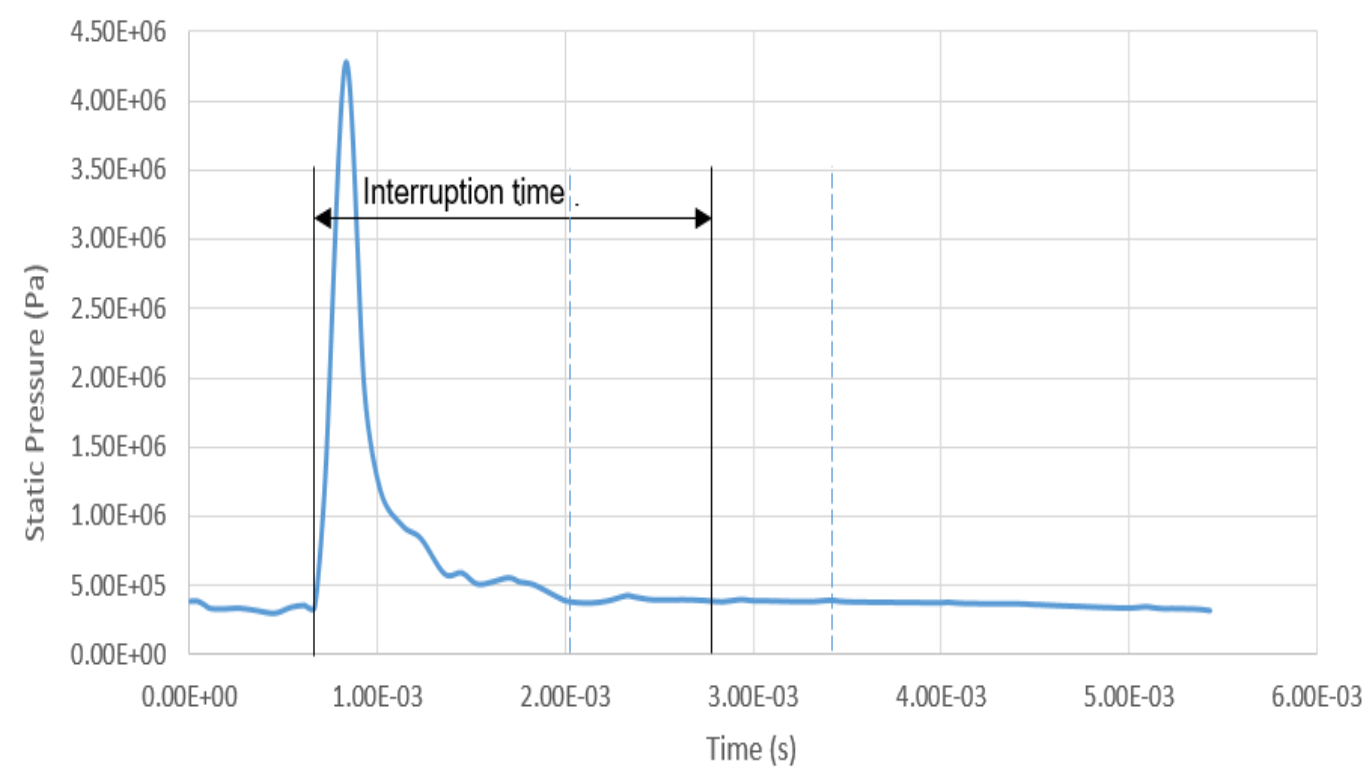

Figure 4.12 Static pressure vs. time at Point 1

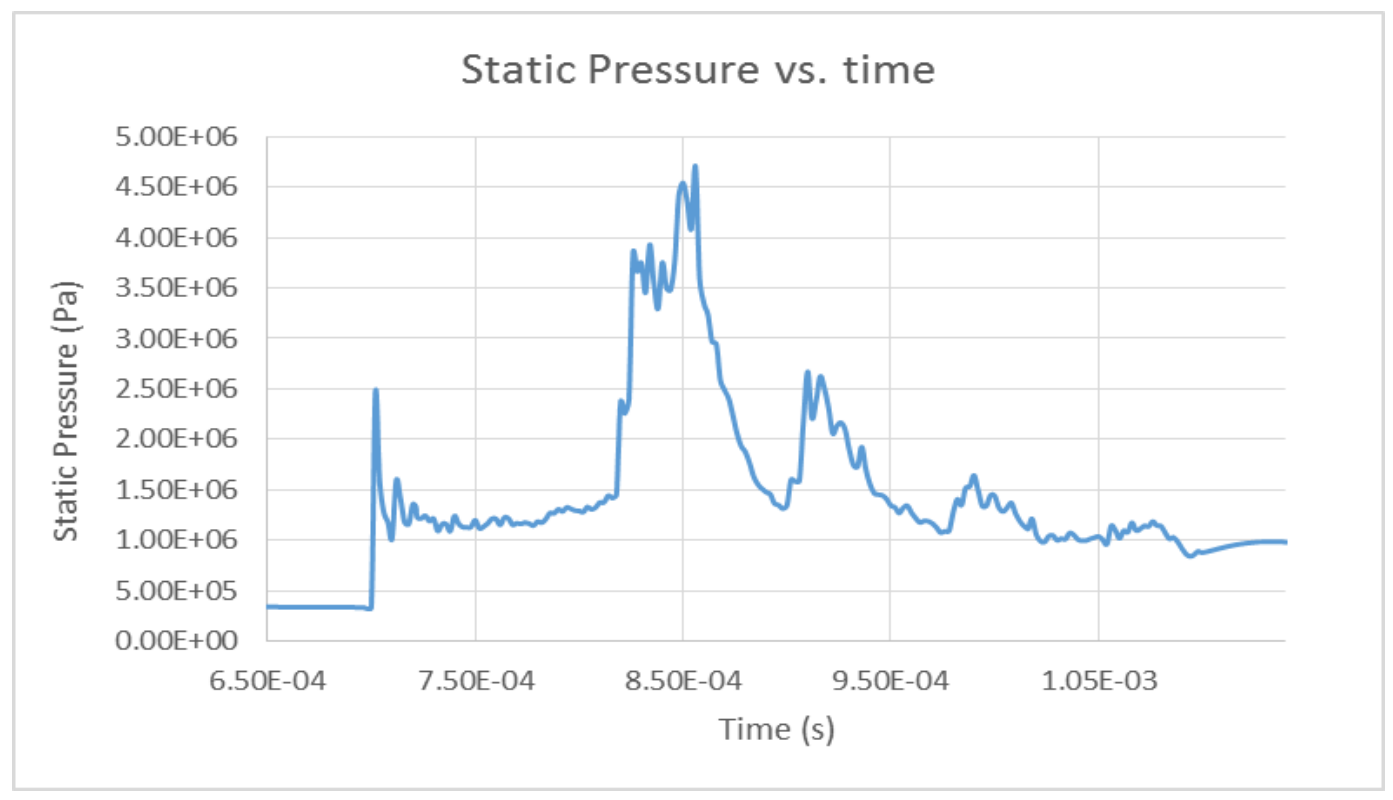

Figure 4.13 Static pressure vs. time at Point 1 showing reflections 
Figure 4.12 can be misleading in the sense that if looks as though there is only reflection inside the cavity. But by studying the same plot on a much smaller time interval, we can see that there multiple reflections within the cavity before the pressure returns to steady state value (Figure 4.13).

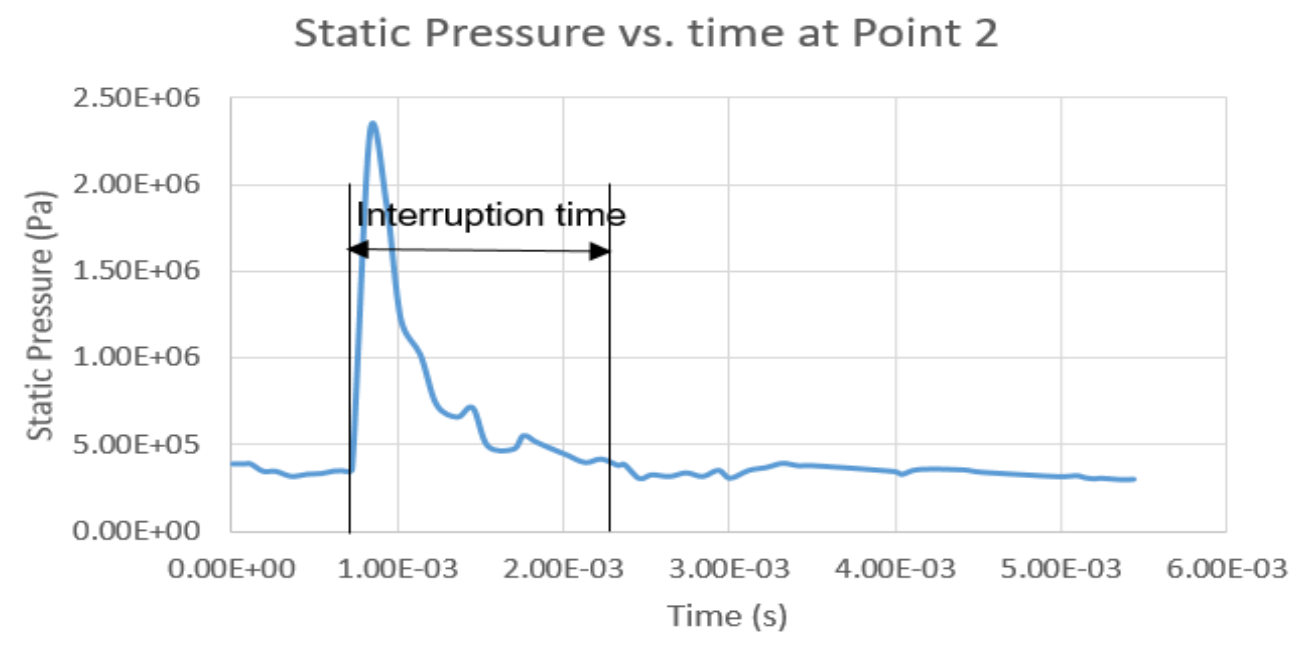

Figure 4.14 Static pressure vs. time at Point 2 


\section{Static Pressure vs. time at Point 3}

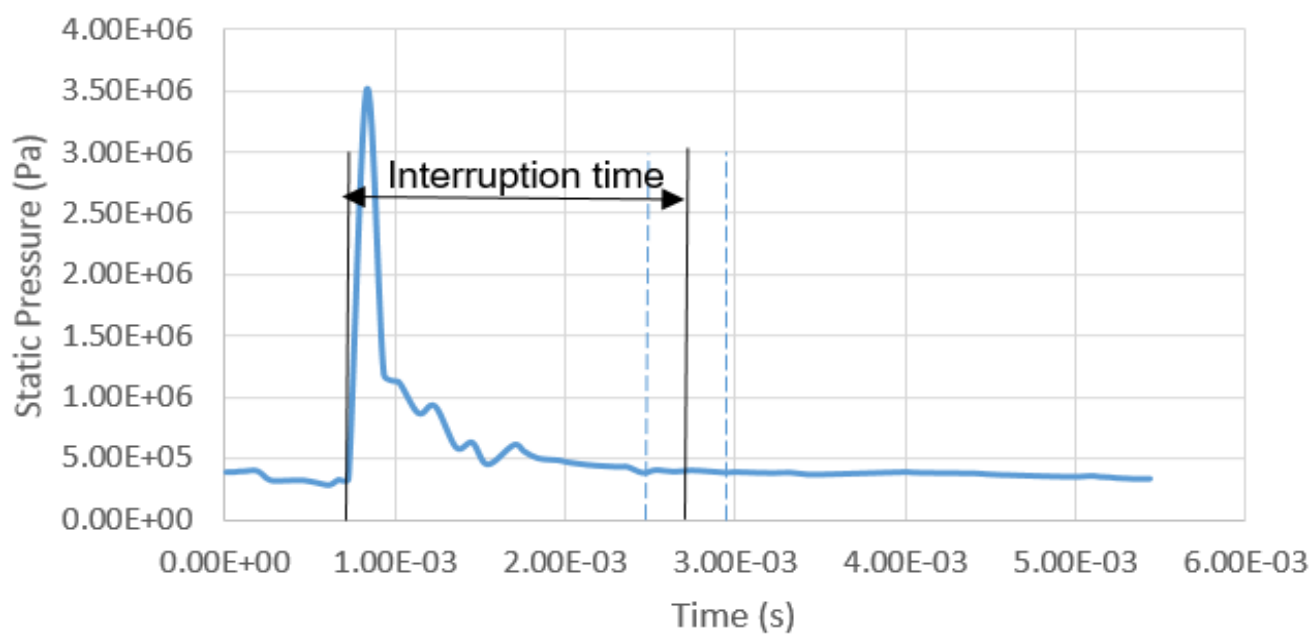

Figure 4.15 Static pressure vs. time at Point 3

The dashed lines in the plots are regions of uncertainty. The first dashed line represents the first point in time when pressure drops to steady state value. A slight fluctuation in pressure is observed until the second dashed line after which pressure almost remains constant. The solid line represents the average value and is used to calculate the time.

At Point 1 , interruption time, $\mathrm{t} 1 \mathrm{int}=(2.76 \mathrm{E}-03-6.65 \mathrm{E}-04)=2.095 \mathrm{~ms}$

At Point 2, interruption time, $\mathrm{t}_{\text {int }}=(2.33 \mathrm{E}-03-7.22 \mathrm{E}-04)=1.608 \mathrm{~ms}$

At Point 3, interruption time, $3_{\mathrm{int}}=(2.69 \mathrm{E}-03-7.22 \mathrm{E}-04)=1.968 \mathrm{~ms}$

Normally, one would expect time to decrease from point 1 to point 3 . While interruption times at points 1 and 3 follow this trend, point 2 takes less time compared to point 3. This is probably because a circulation of combustion products is observed within the cavity. Point 2 is at the center of this circulation region and since the pressure is 
usually low at the center of such a region, it takes lesser time to return to the steady state value.

Velocity magnitude $(\mathrm{m} / \mathrm{s})$
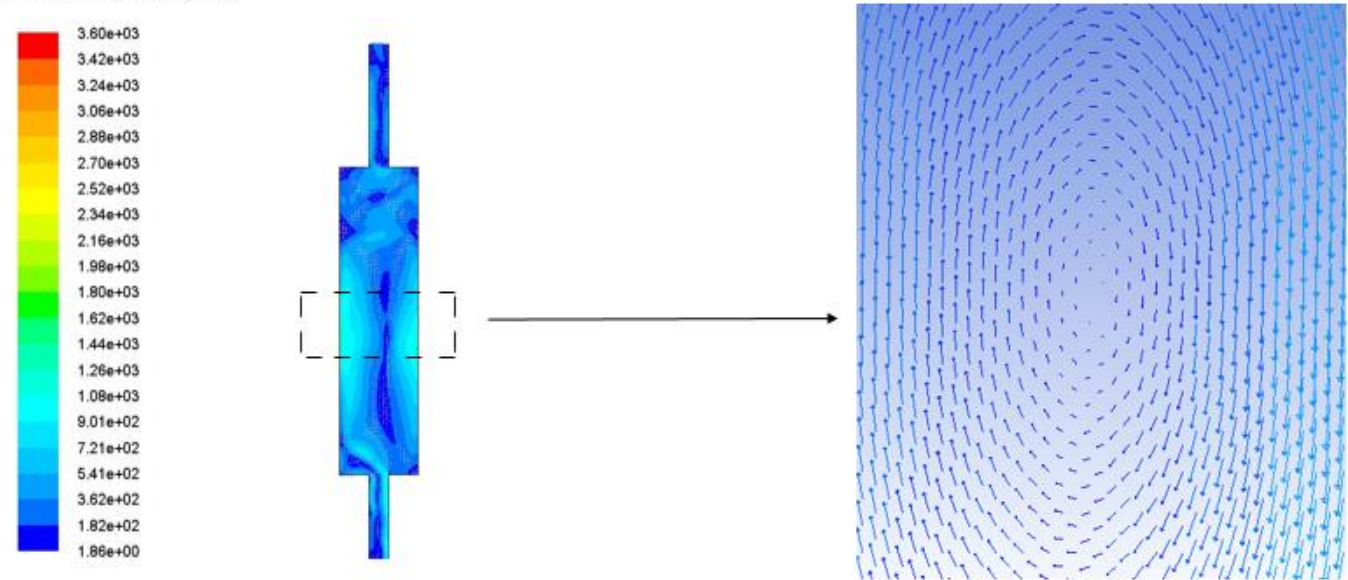

Figure 4.16 Vectors of velocity magnitude 


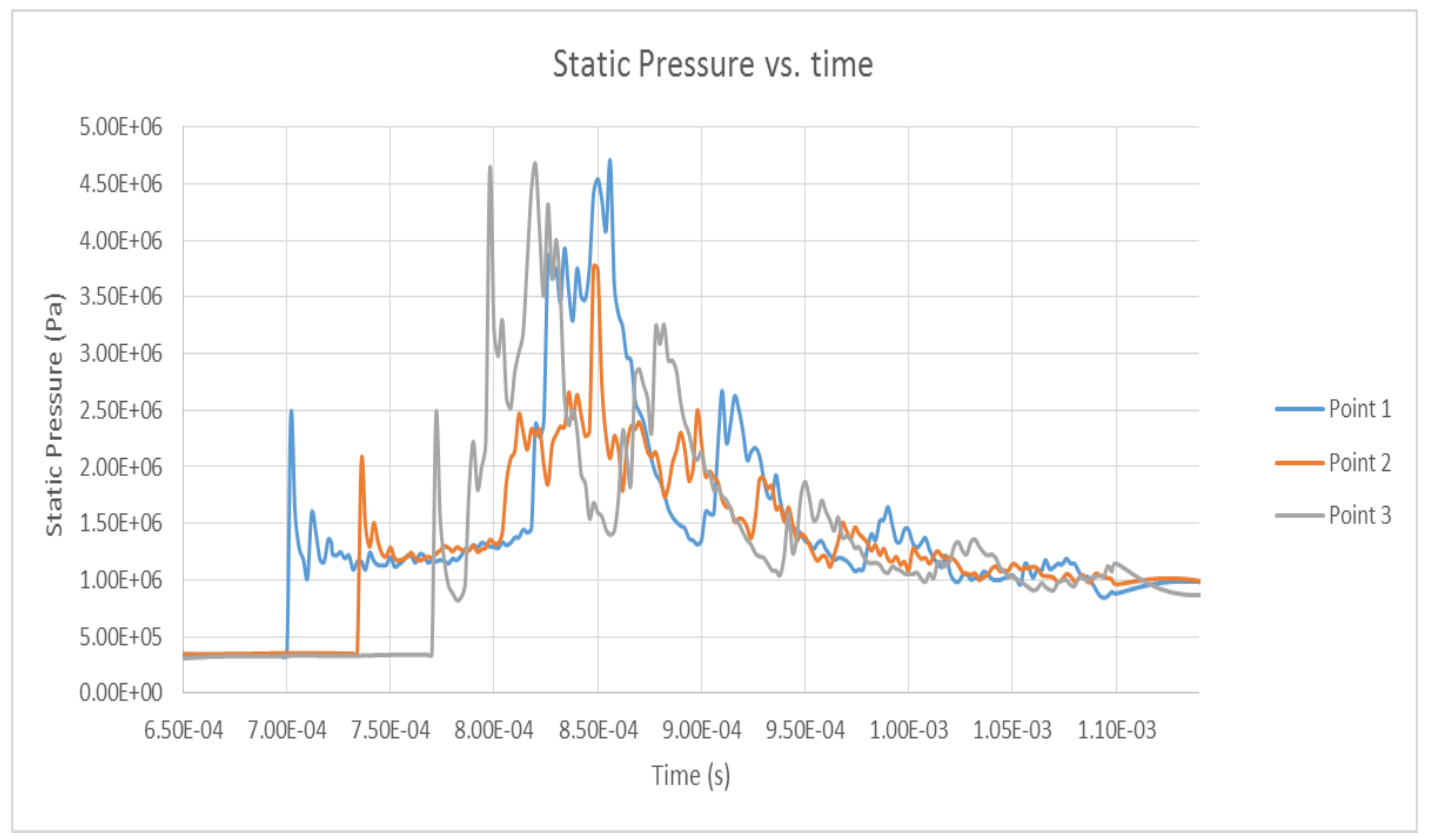

Figure 4.17 Static pressure vs. time indicating multiple reflections within the cavity

The figure above shows the variation in static pressure with respect to time as the shock wave flows into the cavity. The many peaks in the figure indicate multiple shock reflections within the cavity. An $\mathrm{x}-\mathrm{t}$ diagram can be drawn based on this data to determine the location of the shock front. Figure 4.18 is an $x-t$ diagram showing the first reflection. The $\Delta t$ value denoted in the figure is about $0.021 \mathrm{~ms}$, which is much smaller than the interruption time. This also indicates that multiple reflections occur before the pressure in the cavity returns to its original steady state value. The points marked on the graph represent the location of the shock front at a particular instant. The slope of $x-t$ diagram gives the speed of the shock wave.

From the graph, speed of the shock wave, $\mathrm{u}_{\mathrm{s}}=\Delta \mathrm{x} / \Delta \mathrm{t}=555.5 \mathrm{~m} / \mathrm{s}$.

A similar $\mathrm{x}-\mathrm{t}$ diagram showing multiple reflections plotted by Braun for a propane - oxygen detonation with Argon injection is shown in Fig. 4.19. The speed of 
shock wave calculated from this graph gives a value of $544 \mathrm{~m} / \mathrm{s}$. The values differ slightly because the F/O mixtures and injector gases compared are different.

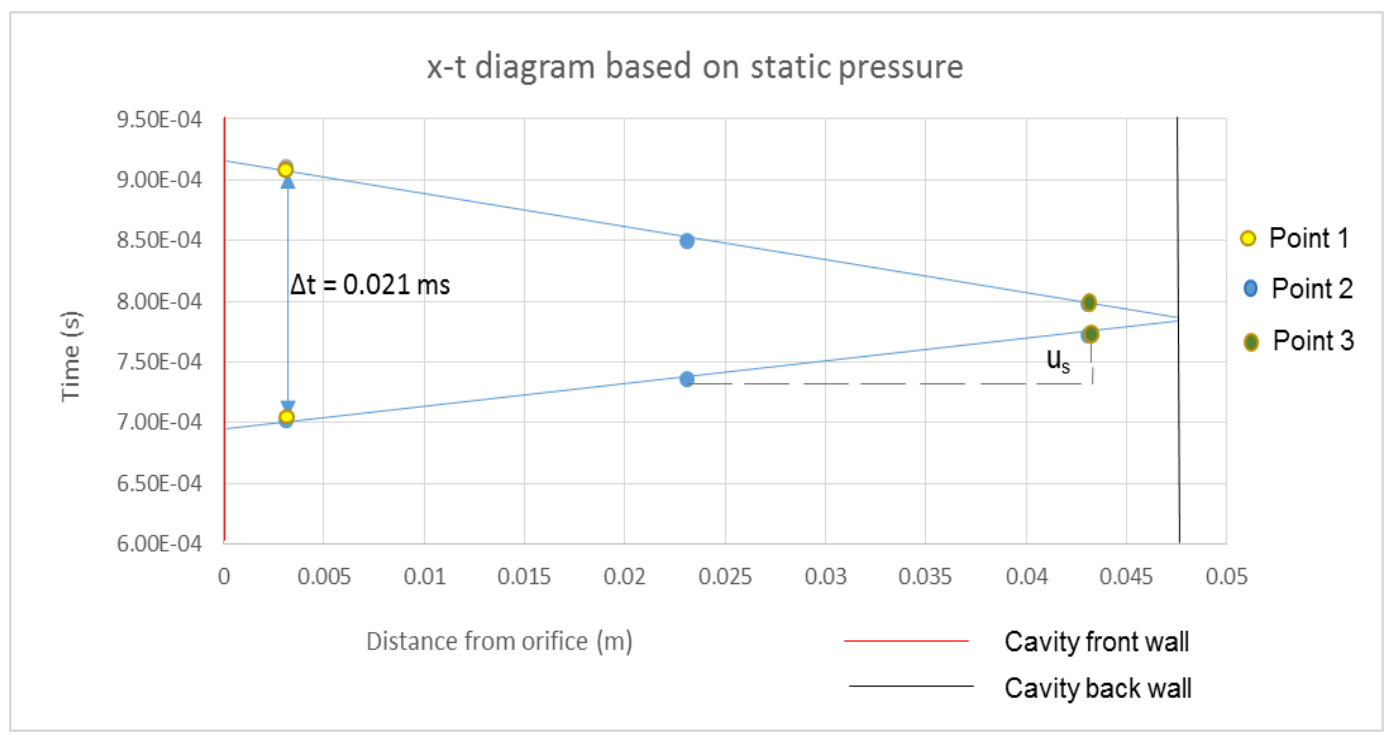

Figure $4.18 \mathrm{x}-\mathrm{t}$ diagram based on static pressure showing first reflection inside the cavity

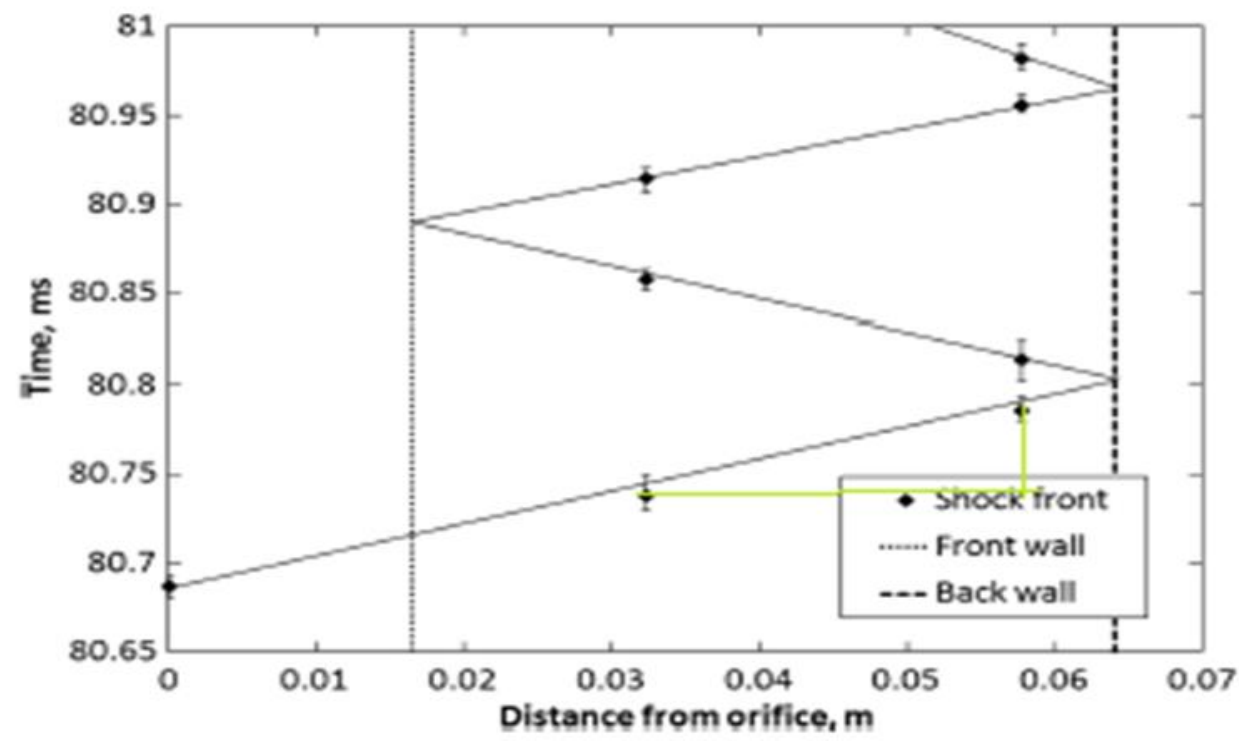

Figure $4.19 x$-t diagram based on experimental values showing multiple reflections ${ }^{[6]}$ 
Another parameter called "valve shut off time" is introduced, which as the name suggests, denotes the time during which the valve is closed. There is no flow of oxygen from the valve into the cavity during this time. This parameter can also be determined graphically by plotting mass fractions of Oxygen as a function of time at points 1,2 and 3 and averaging the values.

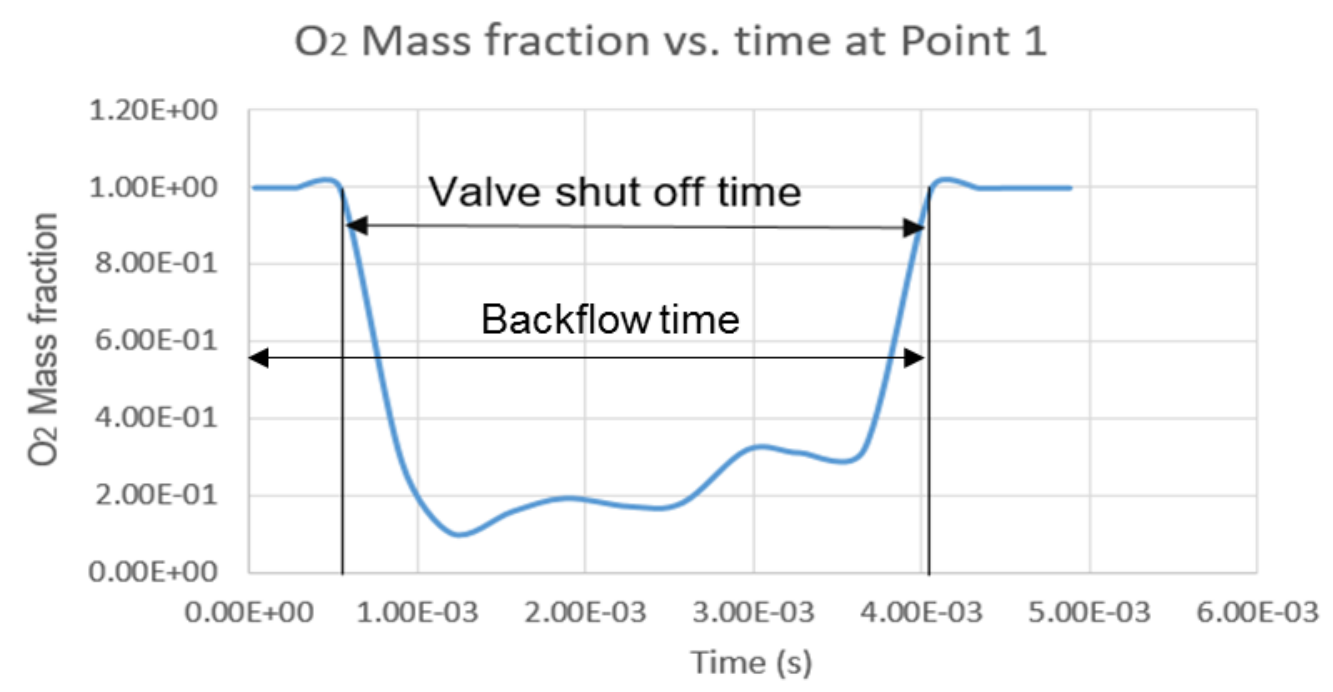

Figure 4.20 Mass fraction of $\mathrm{O}_{2}$ vs. time at Point 1 


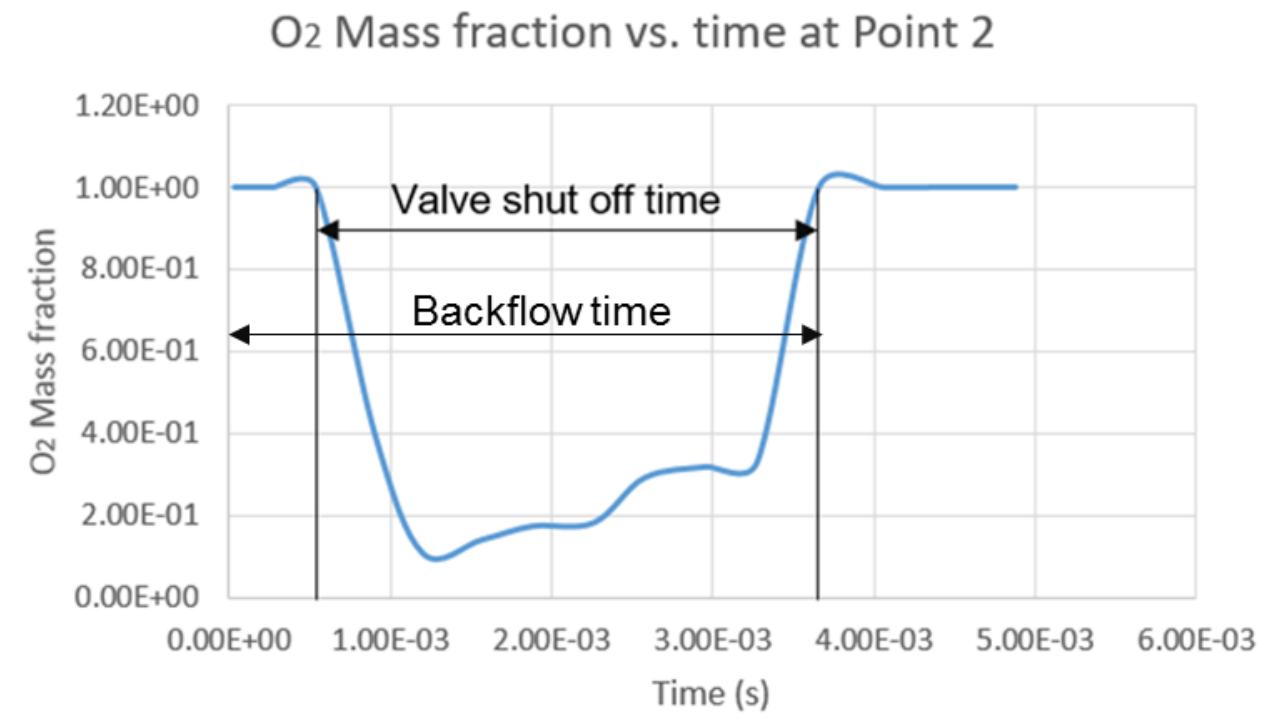

Figure 4.21 Mass fraction of $\mathrm{O}_{2}$ vs. time at Point 2

$\mathrm{O}_{2}$ Mass fraction vs. time at Point 3

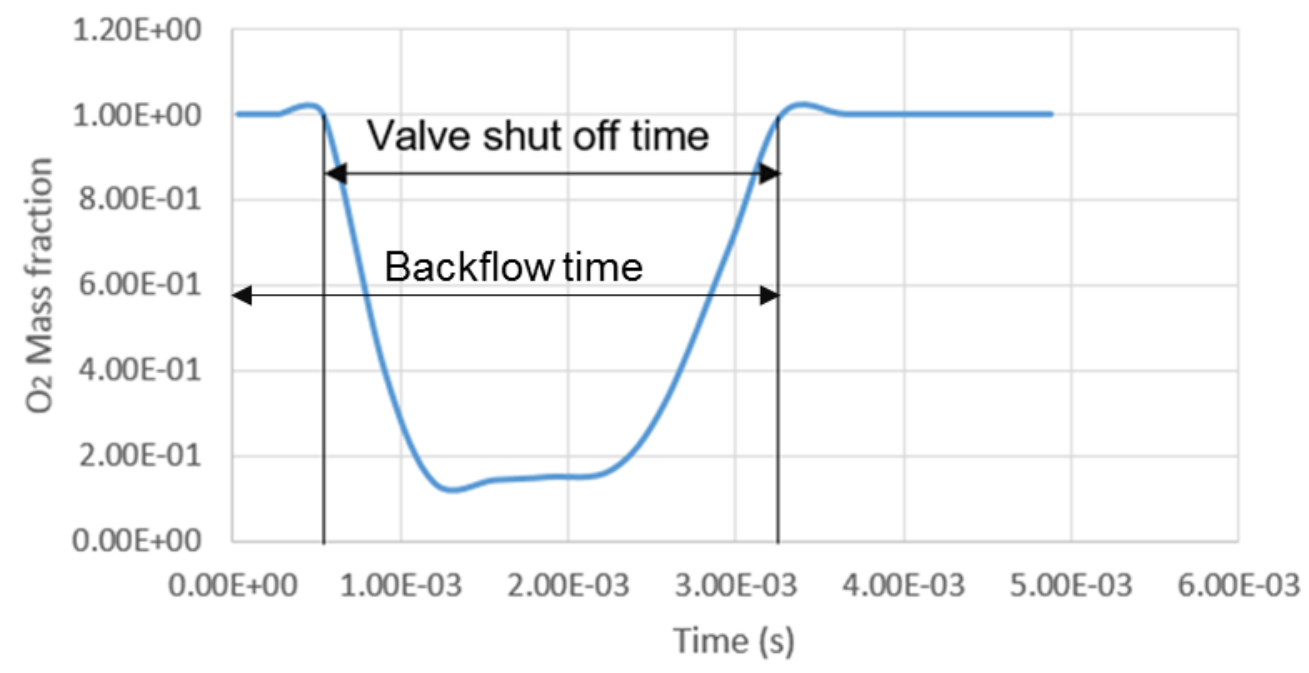

Figure 4.22 Mass fraction of $\mathrm{O}_{2}$ vs. time at Point 3 
At Point 1, valve shut off time, $\mathrm{t} 1_{\mathrm{val}}=(4.06 \mathrm{E}-03-5.4 \mathrm{E}-04)=3.52 \mathrm{~ms}$

At Point 2, valve shut off time, t2val $=(3.66 \mathrm{E}-03-5.4 \mathrm{E}-04)=3.12 \mathrm{~ms}$

At Point 3, valve shut off time, t3val $=(3.28 \mathrm{E}-03-5.4 \mathrm{E}-04)=2.74 \mathrm{~ms}$

Backflow time is defined as the time between start of propellant injection and recommencement of propellant flow after the detonation products have exited the plenum cavity.

At Point 1 , backflow time, $\mathrm{t}_{\mathrm{b}}=(4.06 \mathrm{E}-03-0)=4.06 \mathrm{~ms}$

At Point 2, backflow time, $\mathrm{t}_{\mathrm{b}}=(3.66 \mathrm{E}-03-0)=3.66 \mathrm{~ms}$

At Point 3, backflow time, $\mathrm{t}_{\mathrm{b}}=(3.28 \mathrm{E}-03-0)=3.28 \mathrm{~ms}$

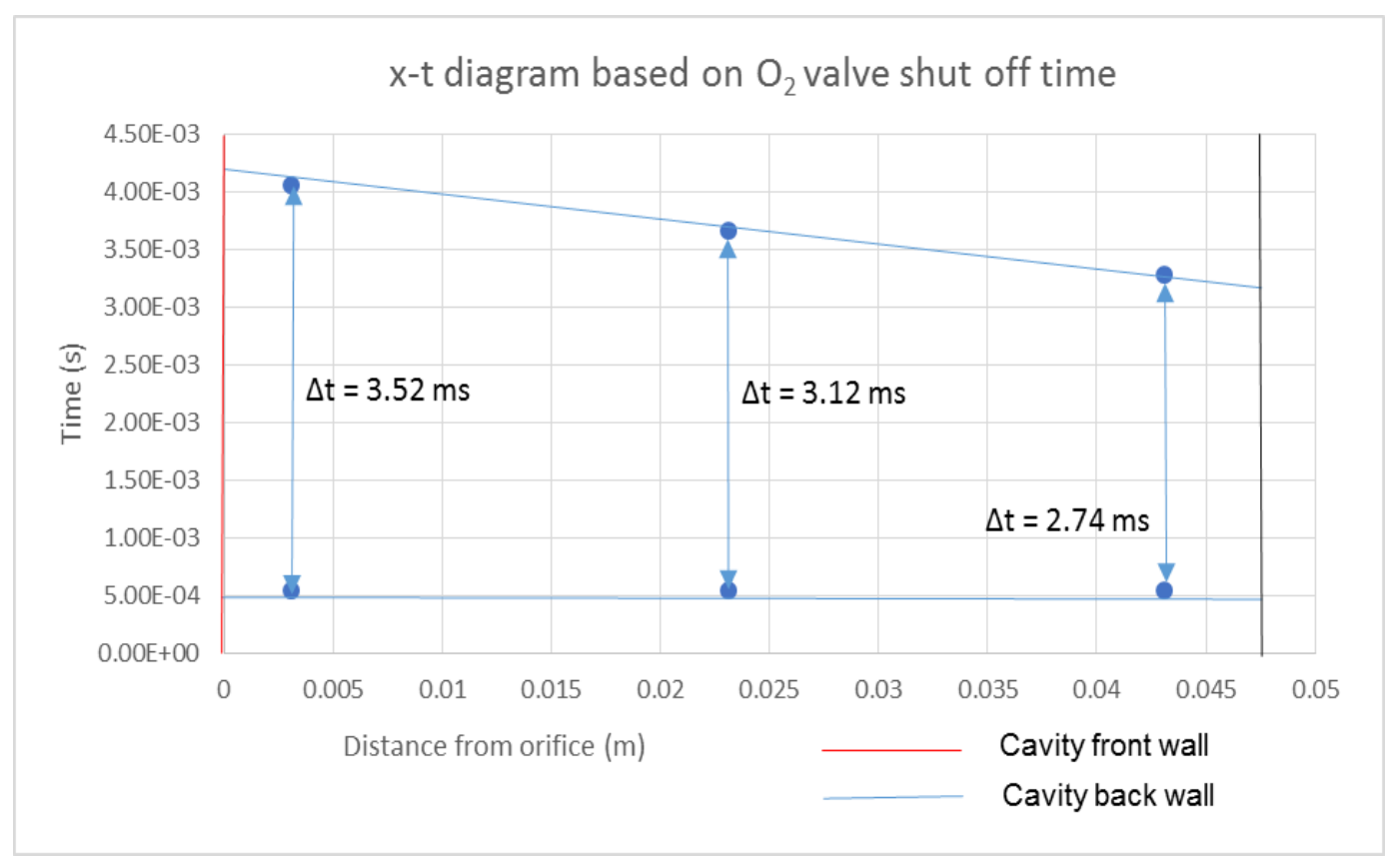

Figure $4.23 \mathrm{x}$-t diagram based on $\mathrm{O}_{2}$ valve shut off time 


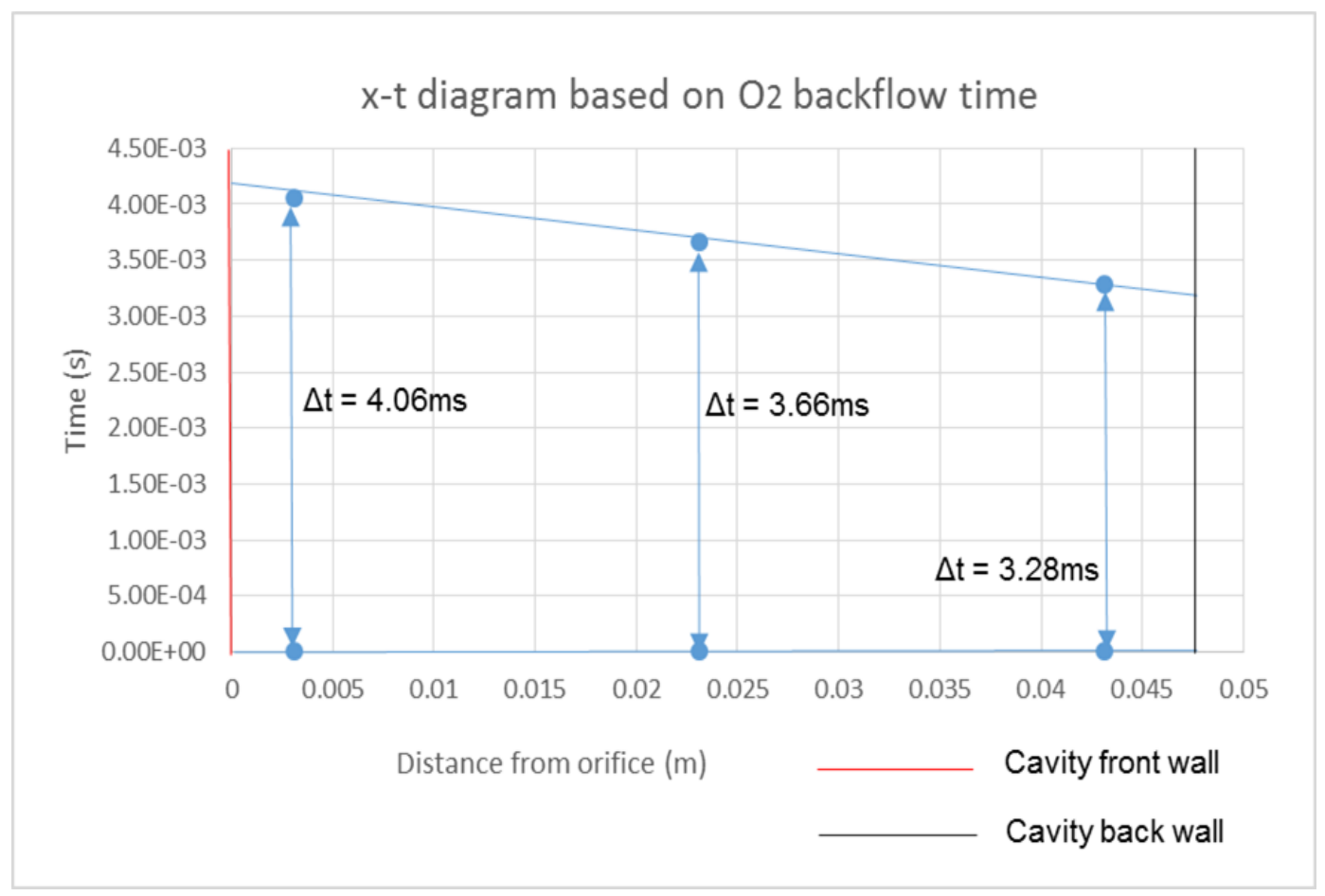

Figure $4.24 \mathrm{x}$-t diagram based on $\mathrm{O}_{2}$ backflow time

Figures 4.23 and 4.24 are $\mathrm{x}-\mathrm{t}$ diagrams based on $\mathrm{O}_{2}$ valve shut off time and backflow time measured at points 1,2 and 3 .

By comparing interruption time and valve shut off time, we understand that steady state flow consists of detonation products as it takes longer for fresh oxygen to flow into the cavity again. This means that the time for the contact surface to exit the cavity is greater than the time for the reflected shock wave to leave the cavity. This statement is well supported by the discussion on interaction between reflected shock wave and contact surface by Zucrow and Hoffman [42].

Density plots are similar to plots of species concentration. This is because a change in density indicates a change in the composition of species. Therefore, by 
analyzing density plots, we can identify the location of contact surface and determine when it leaves the cavity.

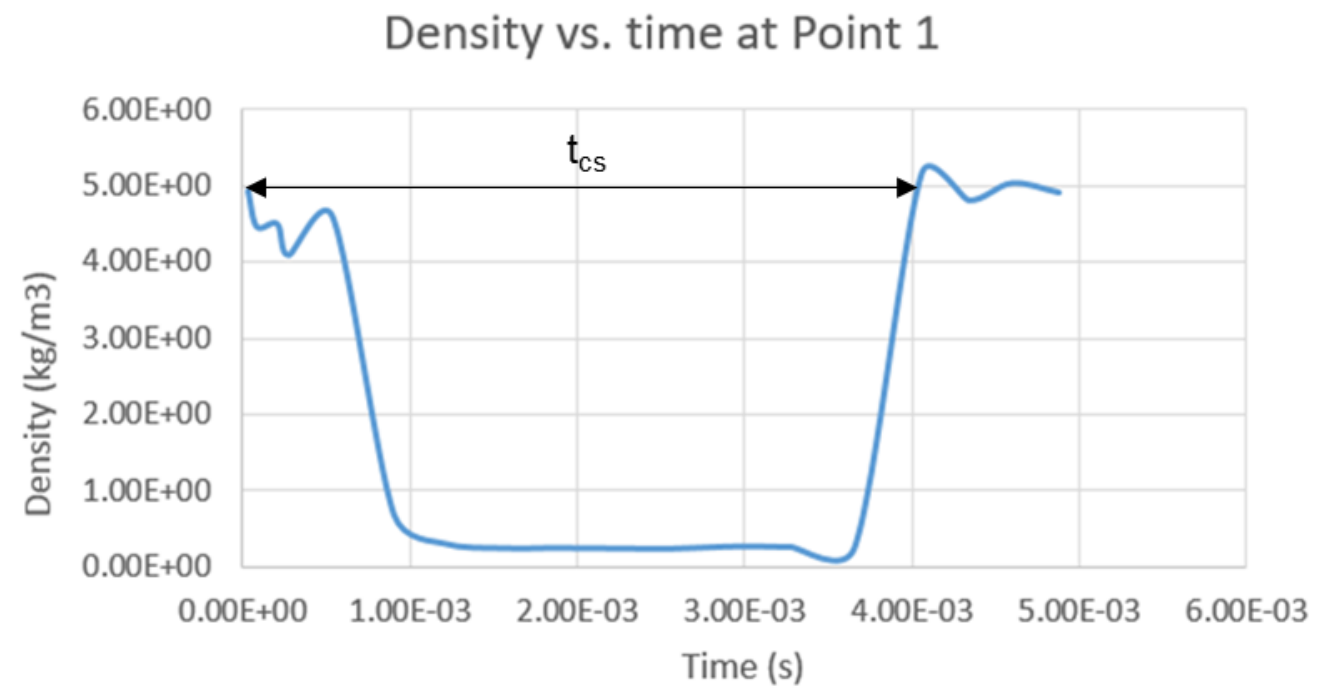

Figure 4.25 Density vs. time at Point 1

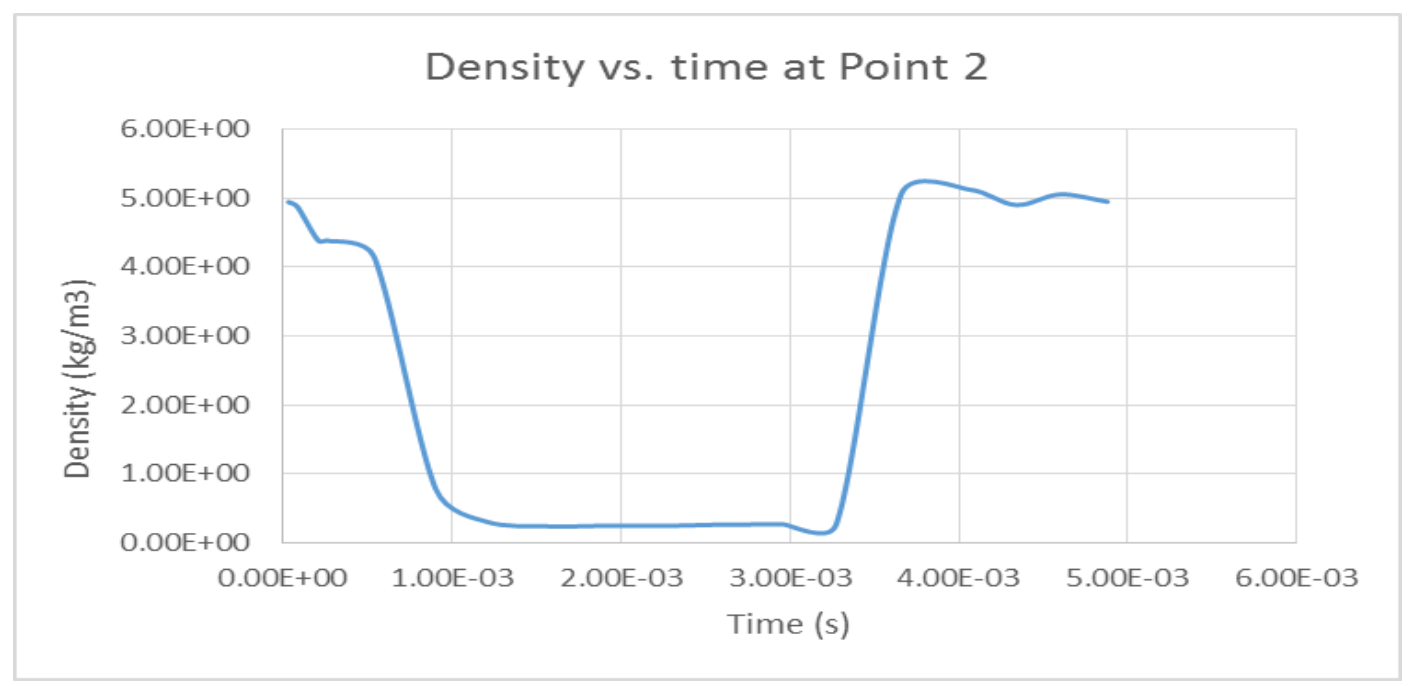

Figure 4.26 Density vs. time at Point 2 


\section{Density vs. time at Point 3}

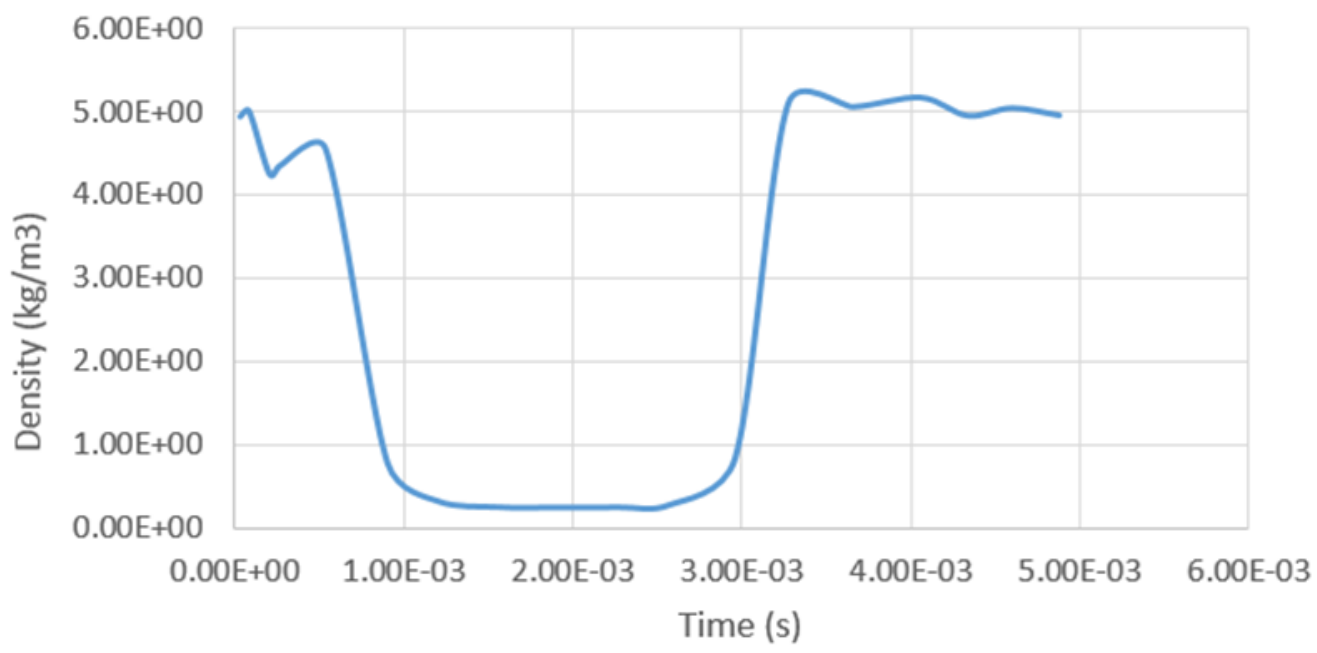

Figure 4.27 Density vs. time at Point 3

Time taken for contact surface to leave the plenum cavity is denoted by $t_{c s}$ in Figure 4.25.

$t_{c s}=4.06 \mathrm{~ms}$, which is the same as backflow time at point 1 . The location of contact surface in the cavity can be determined from the $x$ - t diagram shown in Fig. 4.28. The points on the curve (left to right) correspond to measurements from points 1, 2 and 3 . 


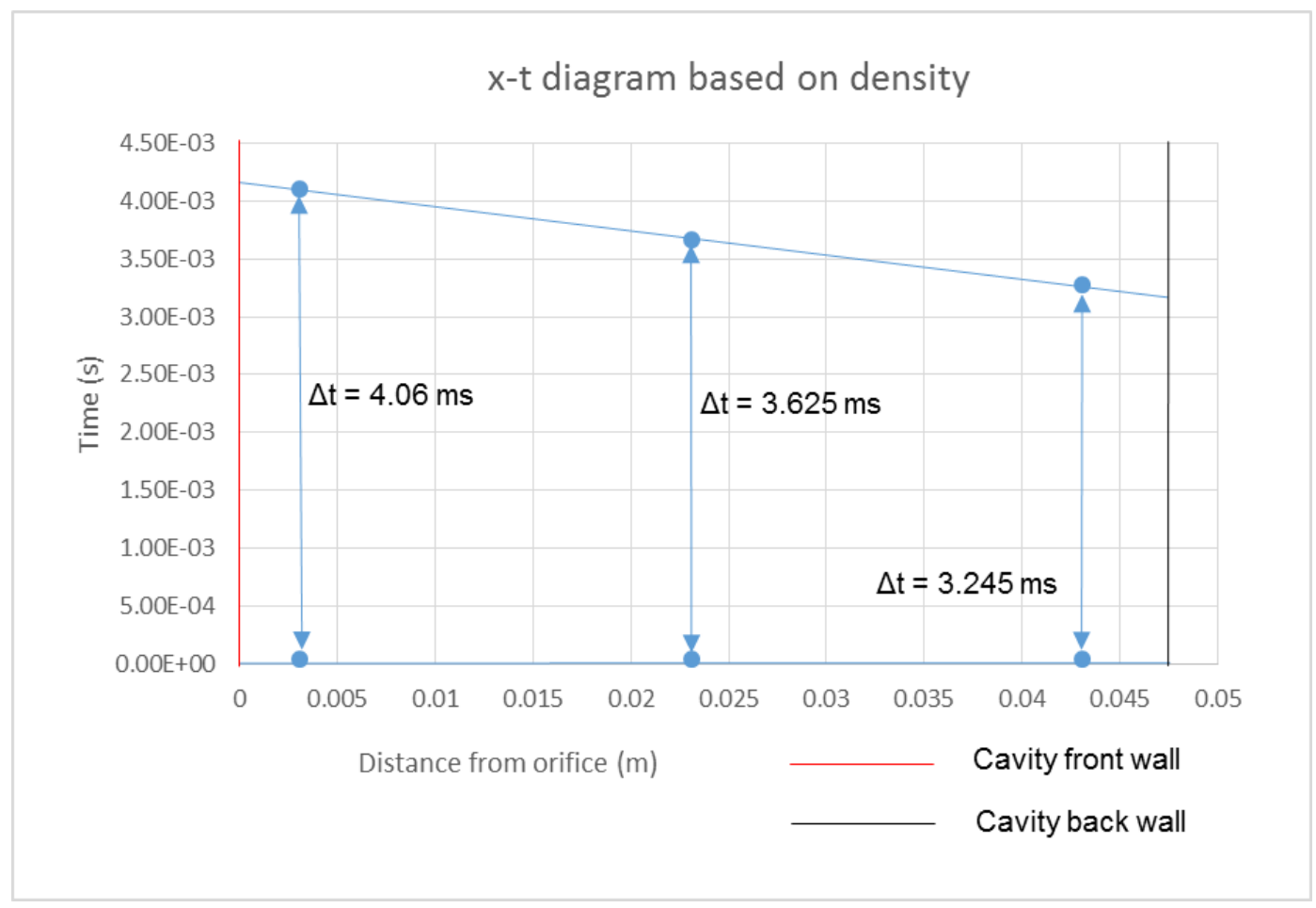

Figure $4.28 x$-t diagram based on density

Temperature plots follow a trend that is approximately the inverse of density plots, as density and temperature have an inverse relation. In the plots below, the cavity is initially at a lower temperature followed by a spike which occurs when the detonation products enter the valve. The temperature drops down to its steady state value when fresh propellants flow back into the cavity thereby replacing hot combustion products. An $\mathrm{x}$-t diagram based on temperature is also plotted. 


\section{Temperature vs. time at Point 1}

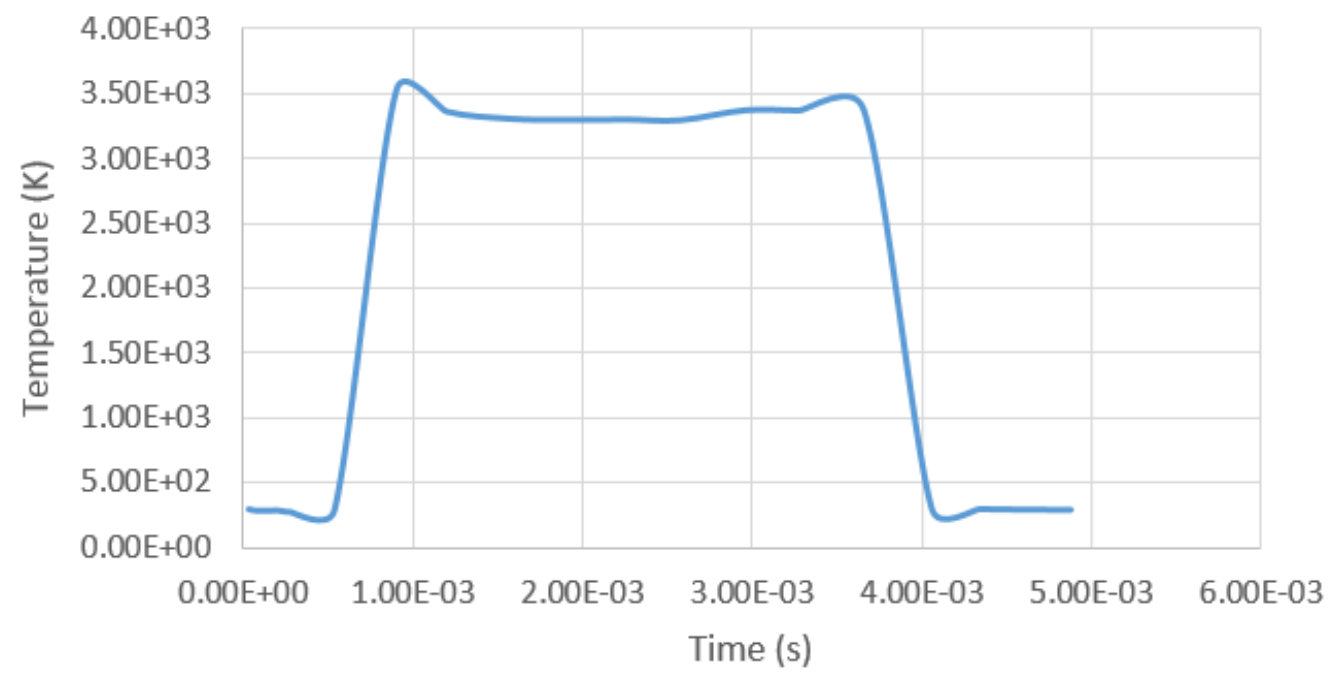

Figure 4.29 Temperature vs. time at Point 1

Temperature vs. time at Point 2

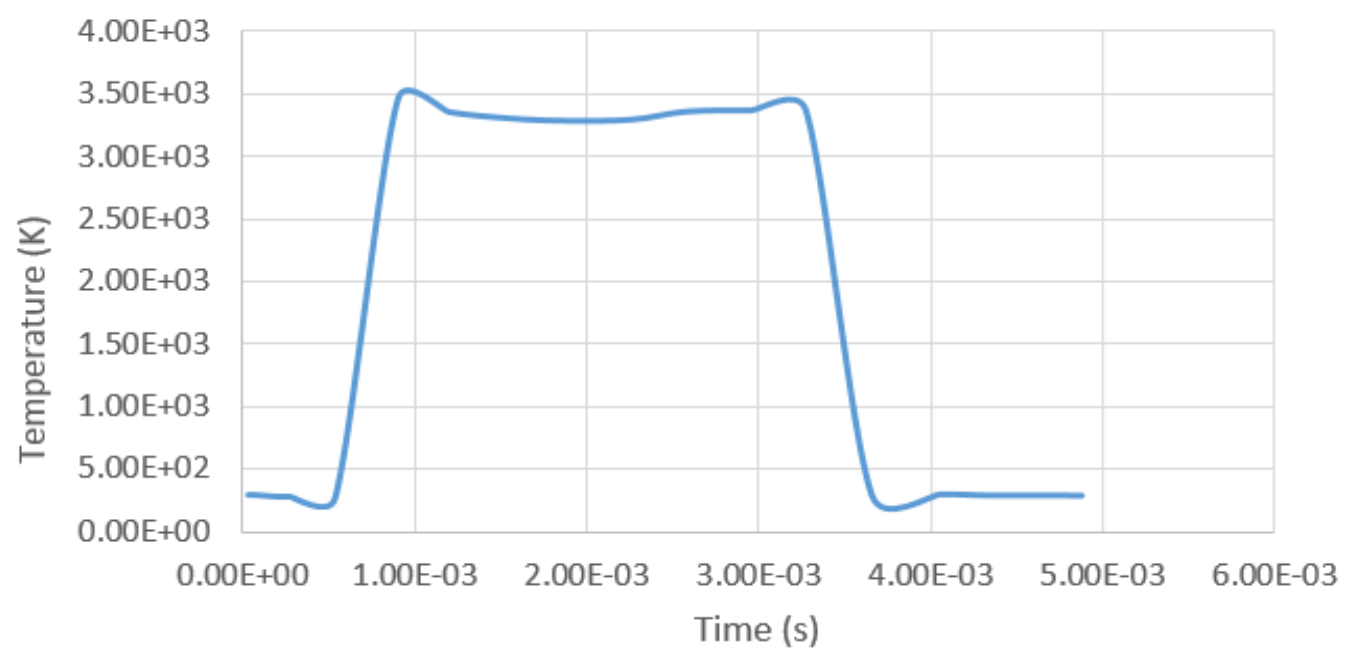

Figure 4.30 Temperature vs. time at Point 2 
Temperature vs. time at Point 3

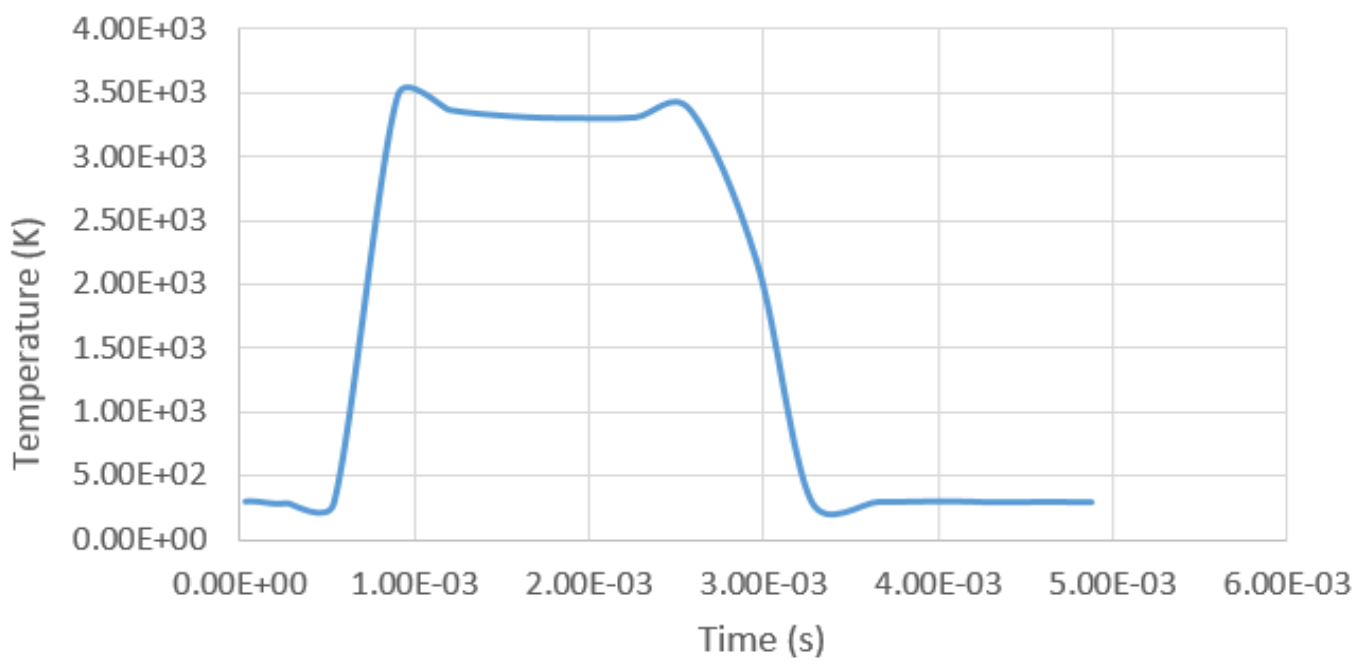

Figure 4.31 Temperature vs. time at Point 3

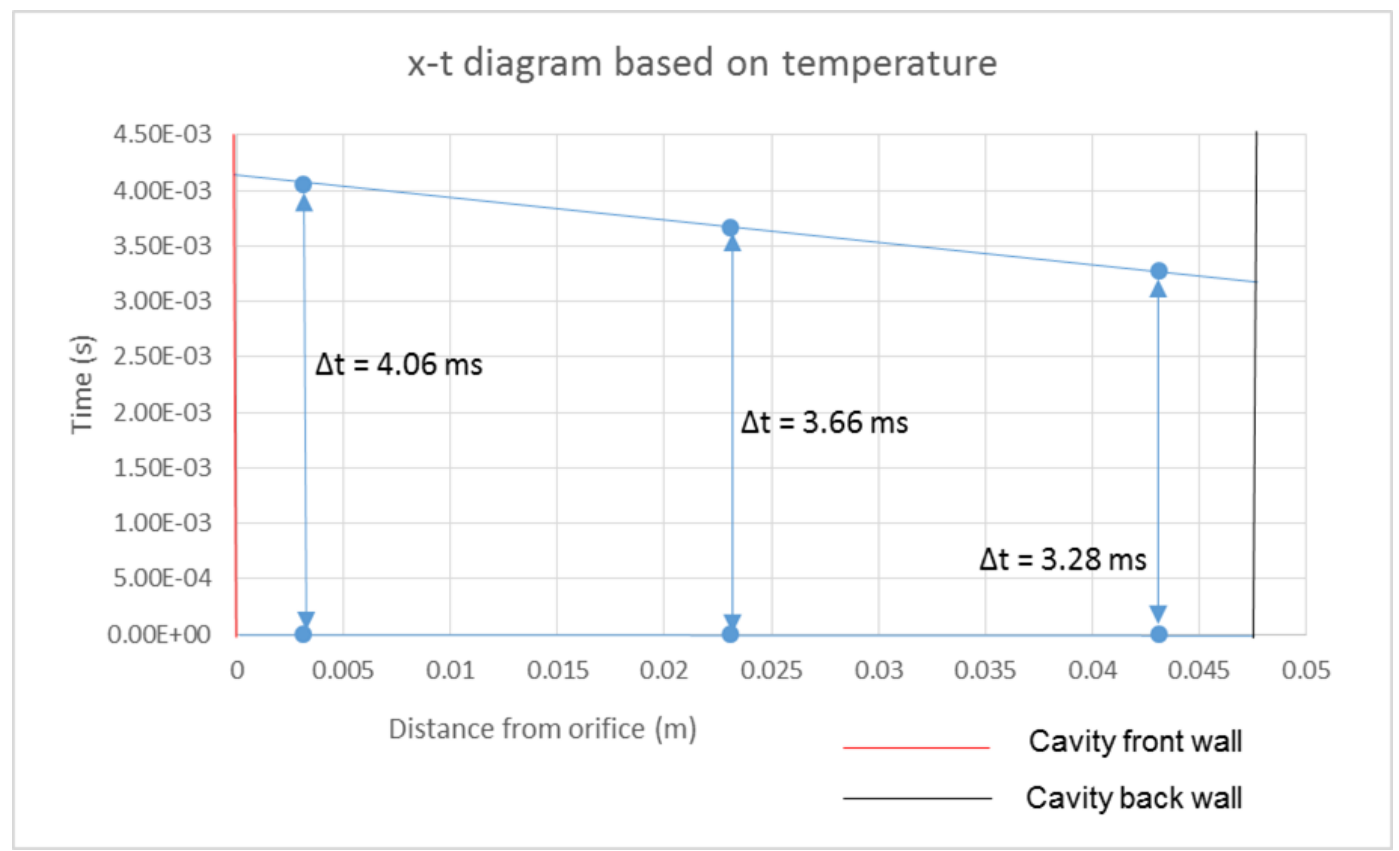

Figure $4.32 \mathrm{x}$-t diagram based on temperature 
It is seen that the valve shut off time obtained from the species plots $\left(\mathrm{O}_{2}\right.$ concentration) is same as the corresponding times obtained from density and temperature plots. Therefore, the species, density and temperature plots indicate a backflow time greater than the interruption time obtained from the pressure plots. It should also be noted that in each of the x-t diagrams shown above, there are multiple reflections within the cavity that occur within the two lines shown.

\subsection{Comparison with experimental results}

When the steady state injection pressure is lowered, the time taken to reach steady state pressure (interruption time) increases as it takes a longer time for the rarefaction wave to reach a lower pressure [6]. This is shown in table 4.1. We have four data points for the $0.32 \mathrm{~cm}$ diameter orifice and $25 \times 0.5 \mathrm{~mm}$ orifice array from the experiment (square markers in Figure 4.26). The steady state pressure in each case is different and corresponding interruption times are noted. The results from CFD analysis (circular marker in Figure 4.26) are then compared with this data to see if it follows the trend.

A comparison based on trend is used to validate the results because the values for hydrogen-oxygen detonation with oxygen injection are not reported in Braun's paper.

Behavior of argon and oxygen as injector gases can be compared due to similar speeds of sound. Although the F/O mixtures compared in the table below are different, primary focus is on the relation between steady state pressure and interruption time. Nevertheless, a non-dimensional pressure parameter was introduced to confirm this. 
Propane - oxygen and hydrogen - oxygen have different vales of CJ pressure. This value of pressure is divided by the steady state pressure in each case to form a nondimensional pressure parameter. A plot of this parameter versus interruption time (Figure 4.33) is plotted and it proves that the trend followed by both the mixtures are similar.

Table 4.1 Comparison with experimental results

\begin{tabular}{|l|l|l|l|l|}
\hline Analysis & F/O mixture & Injector gas & $\begin{array}{l}\text { Steady State } \\
\text { Pressure (atm) }\end{array}$ & tint (ms) \\
\hline Experiment & Propane/Oxygen & Argon & 13.8 & 0.881 \\
\hline Experiment & Propane/Oxygen & Argon & 9.5 & 1.61 \\
\hline Experiment & Propane/Oxygen & Argon & 6.12 & 1.87 \\
\hline CFD & Hydrogen/Oxygen & Oxygen & 3.8 & 1.968 \\
\hline Experiment & Hydrogen/Oxygen & Oxygen & 2.7 & 2.3 \\
\hline
\end{tabular}

In the table above, only the interruption time determined at point 3 is used for comparison. This is due to the fact that the pressure values obtained experimentally were measured with a pressure transducer located in the cavity at a point same as point 3 .

It is evident from the table that the result obtained from CFD follows the trend mentioned earlier. 


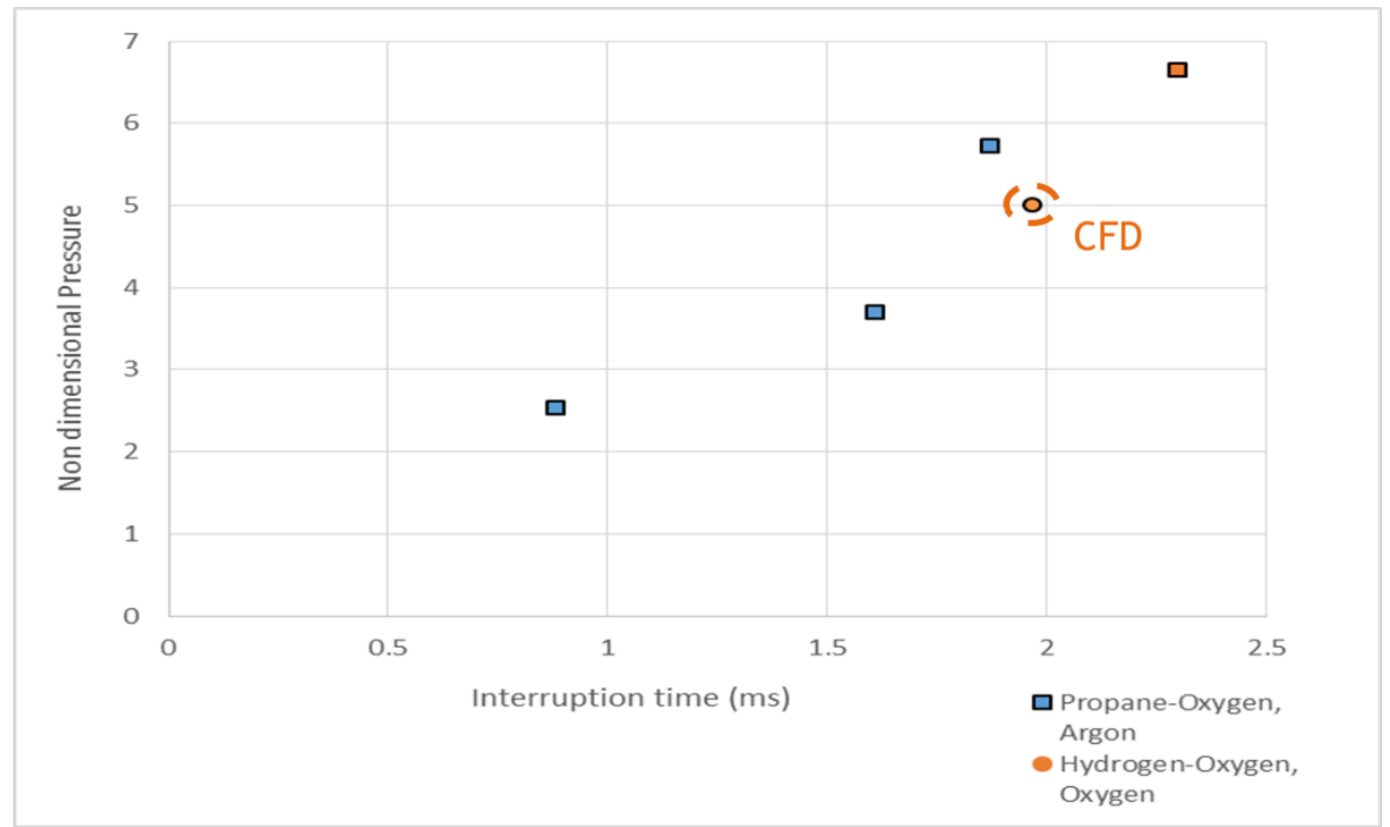

Figure 4.33 Plot of Non dimensional pressure vs. Interruption time 
Chapter 5

Conclusion

The CFD analysis conducted in this study is an extension to the experimental work done by Braun. $\mathrm{A} \mathrm{H}_{2}-\mathrm{O}_{2}$ detonation was studied using a 19 step combustion mechanism in a $3.05 \mathrm{~m}$ long tube with fluidic valves serving as injectors. The valve had an orifice diameter of $0.32 \mathrm{~cm}$ and a plenum cavity operating at a constant pressure of $3.8 \mathrm{~atm}$; one of the configurations and conditions tested in the experiment. While experiments could only predict the interruption time, CFD analysis was required to see if steady state flow was comprised of combusted products or fresh propellants. Therefore, interruption time and valve shut off time were determined and compared using pressure, density and species plots. Results suggest that the return to steady state flow based on pressure measurements does not coincide with resumption of oxygen flow through the cavity. The flow within the cavity between the end of the interruption time and the valve time is still comprised of detonation products as it takes a longer time for the contact surface to exit the cavity and for fresh propellants to flow back. In other words, valve shut off time is more than the interruption time.

The advantage of developing detonation engines with fluidic valves as injectors is to realize operating frequencies higher than $100 \mathrm{~Hz}$.

Operating frequency of the detonation engine studied can be calculated as:

$1 /$ valve shut off time at point $1=1 / 0.00352 \mathrm{~s}=284.1 \mathrm{~Hz}$

Operating frequency of the detonation engine based on interruption time at point 1 is:

$1 / 0.002095=477.3 \mathrm{~Hz}$. 
Although calculation based on interruption time predicts a higher operating frequency than valve shut off time, it is still higher than the operating limit of conventional mechanical valves (about $100 \mathrm{~Hz}$ ). This suggests that fluidic valves can be used in high frequency detonation engines.

This work can therefore be used as a tool to design valves in RDEs and to refine orifice geometries and dimensions to achieve much higher operating frequencies. 
Chapter 6

Future work

The current analysis is based on the assumption that the flow is inviscid. Although such a model predicts detonation properties fairly accurately, turbulence and molecular diffusion are known to play a key role in detonation. Therefore, viscous effects can be included in future work.

This study is limited to hydrogen - oxygen detonation and a single kind of orifice geometry. By extending this work to various fuel - oxidizer combinations and different kinds of orifices, we can observe how valve time changes with propellants and predict the best combination of $\mathrm{F} / \mathrm{O}$ mixture and orifice geometry. This will also help in designing valves in RDEs capable of operating at much higher frequencies.

Although varying grid sizes have been used in this model to save time and resources, it has been performed manually. By performing Adaptive Mesh Refinement in future, computation time and resources can be reduced drastically! 
References

[1] https://en.wikipedia.org/wiki/History_of_aviation

[2] https://en.wikipedia.org/wiki/Pulse_detonation_engine

[3] Raghupathy, A., "A Numerical Study of Detonation and Plume Dynamics in a Pulsed Detonation Engine," Masters Thesis, Dept. of Mechanical, Industrial and Nuclear Engineering, University of Cincinnati., Ohio, 2005.

[4] Musielak, Dora . E., "Fundamentals of Pulse Detonation Engine (PDE) and Related Propulsion Technology," University of Texas at Arlington, June 2014.

[5] Fickett, W., and Davis, W.C., "Detonation," University of California Press, 1979.

[6] Braun, E., "New Detonation Concepts for Propulsion and Power Generation," PhD Dissertation, Dept. of Mechanical, and Aerospace Engineering, University of Texas at Arlington, Arlington, TX 2012.

[7] Bulata, P. V., and Volkovb, K. N., "Detonation Jet Engine. Part 1 - Thermodynamic Cycle," International Journal of Environmental \& Science Education, Vol. 11, No. 12, 2016, 5009-5019.

[8] E, Wintenberger., and Shepherd, J.E., "Thermodynamic Cycle Analysis for Propagating Detonations," Journal of Propulsion And Power, Vol. 22, No. 3, May-June 2006.

[9] Bellini, R., "Ideal Cycle Analysis of a Regenerative Pulse Detonation Engine for Power Production," PhD Dissertation, Dept. Of Mechanical, and Aerospace Engineering, University of Texas at Arlington, Arlington, TX 2010.

[10] Rouser, K., King, P., Schauer, F., Sondergaard, R., and Hoke, J., "Experimental Performance Evaluation of a Turbine Driven by Pulsed Detonations," 51st AIAA Aerospace Sciences Meeting, AIAA 2013- 1212, January 2013. 
[11] Schwer, D.A., and K., Kailasanath, "Numerical Investigation of Rotating Detonation Engines," Joint Propulsion Conference, AIAA 2010-6880, July 2010.

[12] Braun, E. M., Balcazar, T. S., Wilson, D. R., and Lu, F. K., "Experimental Study of a High-Frequency Fluidic Valve Fuel Injector," Journal of Propulsion and Power, Vol. 28, No. 5, September-October 2012.

[13] Zel'dovich, Y. B., "K teori rasprostranenia detonazi v gasoobrasnikh systemakh," Zhurnal Experimental'noi i Teoreticheskoi Fiziki, Vol. 10, pp. 543-568, 1940.

[14] von Neumann, J., "Theory of Detonation Waves. Progress Report to the National Defense Research Committee Div. B, OSRD-549, (April 1, 1942. PB 31090)," in John von Neumann: Collected Works, 1903-1957, Vol. 6, A. H. Taub, Ed. New York: Pergamon, 1963.

[15] D“oring, W., "Uber den detonationsvorgang in gasen,” " Annalen der Physik, Vol. 43, pp. 421-436, 1943.

[16] Arienti, M., "A Numerical and Analytical Study of Detonation Diffraction," PhD Dissertation, California institute of Technology, Pasadena, 2003.

[17] Hsu, K., and Jemcov, A., "Numerical Investigation of Detonation in Premixed Hydrogen-Air Mixture - Assessment of Simplified Chemical Mechanisms," AIAA Paper 00-2478, Denver, CO, 2000.

[18]http://3.bp.blogspot.com/_yQmQ1ZvYAok/THPVjdzAYQI/AAAAAAAAB5I/IW2V2D sWLol/s1600/hugoniotcurve.jpg

[19] Zhang, Bo, and ChunHua, Bai, "Research progress on the dynamic characteristics of gaseous detonation," Sci Sin-Phys Mech Astron, Vol. 44: 665-681, 2014.

[20] W, Fickett ., and Wood, W. W., "Flow Calculations for Pulsating One-Dimensional Detonations," Physics of Fluids, Vol. 9, 1966. 
[21] Erpenbeck, J. J., "Stability of Idealized One-Reaction Detonations," Physics of Fluids, Vol. 7, 1964.

[22] Oran, E. S., Weber, J. B.Jr., Stefaniw, E. I., Lefebvre, M. H., and Anderson, J. D.Jr., "A Numerical Study of a Two-Dimensional H2-O2-Ar Detonation Using a Detailed Chemical Reaction Model," Combustion And Flame 113:147-163, 1998.

[23] Sharpe, G. J., and Falle, S. A. E. G., "One-Dimensional numerical simulations of idealized detonations," Proc. R. Soc. A 455 1203-1214, 1999.

[24] Sharpe, G. J. "Linear stability of idealized detonations." Proc. R. Soc. Lond. A453, 2603- 2625, 1997.

[25] He, L., and Lee, J. H. S., "The dynamical limit of one-dimensional detonations," Physics of Fluids, Vol. 7, 1151-1, 1995.

[26] Li, C., Kailasanath, K., and Patnaik, G., "A Numerical Study of Flow Field Evolution in a Pulsed Detonation Engine," AIAA Paper 2000-0314, $38^{\text {th }}$ AIAA Aerospace Sciences Meeting and Exhibit, Reno, NV, 2000.

[27] Hsu, K., and Jemcov, A.,"Numerical Investigation of Detonation in Premixed Hydrogen-Air Mixture - Assessment of Simplified Chemical Mechanisms," AIAA Paper 00-2478, Denver, CO,2000.

[28] Law, C, K., Sung, C, J., Wang, H., and Lu, T, F., "Development of Comprehensive Detailed and Reduced Reaction Mechanisms for Combustion Modeling ," AIAA 2002-0331, $40^{\text {th }}$ AIAA Aerospace Sciences Meeting and Exhibit, Reno, NV,2000.

[29] S. Yungster, and K. Radhakrishnan, "Structure and Stability of One-Dimensional Detonations in Ethylene-Air Mixtures," 33rd AIAA Fluid Dynamics Conference, Orlando, Florida, 23-26 June 2003. 
[30] Bourlioux, A., Majda, A.J. and Roytburd, V., "Theoretical and Numerical Structure of Unstable One-Dimensional Detonations," SIAM Journal of Applied Mathematics, Vol. 51, pp. 303-343, April 1991.

[31] Fedor A. Bykovskii, Sergey A. Zhdan, and Evgenii F. Vedernikov, "Continuous Spin Detonations," Journal of Propulsion and Power, Vol. 22, No. 6, NovemberDecember 2006.

[32] Baklanov, D. I., Golovastov, S. V., Golub, V. V., Reshetnyak, R. B.,Semin, N. V., and Volodin, V. V., "Model of Low-Thrust Pulsed Detonation Device with Valveless Fuel Feed," 2nd European Conference for Aerospace Sciences (EUCASS) [CD-ROM], Brussels,Univ. Libre de Bruxelles/von Kármán Inst. for Fluid Dynamics,Brussels/Flemish Brabant, Belgium, 2007.

[33] Matsuoka, Ken., Yageta, Jun., Nakamichi, Tatsuya., Kasahara, Jiro., Yajima, Takashi., and Kojima, Takayuki., "Inflow-Driven Valve System for Pulse Detonation Engines," Journal Of Propulsion And Power, Vol. 27, No. 3, May-June 2011.

[34] Liu, Shi-Jie., Lin, Zhi-Yong., Liu, Wei-Dong., Lin, Wei., and Sun, Ming-Bo., "Experimental and three-dimensional numerical investigations on $\mathrm{H} 2$ /air continuous rotating detonation wave," Journal of Aerospace Engineering, 2011.

[35] ANSYS Fluent Theory Guide, Release 17.0, ANSYS Inc., Canonsburg, PA, 2016.

[36] G.D. Smith., Numerical solution of partial differential equations, $2^{\text {nd }}$ ed., New York, 1979.

[37] Wada, Y., and Liou, M.-S., "An Accurate and Robust Flux Splitting Scheme for Shock and Contact Discontinuities," SIAM Journal of Scientific Computing, Vol. 18, 633657, 1997.

[38] Wilson, G. J., and MacCormack, R. W., "Modeling Supersonic Combustion Using a Fully Implicit Numerical Method," AIAA Paper 90-2307, July 1990. 
[39] Peace, J., and Lu, F. K., "Numerical Study of Pulse Detonation Engine Nozzle and Exhaust Flow Phenomena," 51st AIAA/SAE/ASEE Joint Propulsion Conference, July 27-29, 2015, Orlando, FL.

[40] Raghupathy, Arun Prakash., Ghia, Urmila., and Ghia, Karman.," A Comparative Study of Chemical Mechanisms in the Simulation of One-Dimensional Detonation," 42nd AIAA/ASME/SAE/ASEE Joint Propulsion Conference \& Exhibit, 9 - 12 July 2006, Sacramento, California

[41] Gordon, S., McBride, B.J.: Computer Program for Calculation of Complex Chemical Equilibrium Compositions andApplications. I. Analysis, NASA Reference Publication 1311, 1994.

[42] Zucrow, M. J., and Hoffman, J. D., "The Method of Characteristics Applied to Unsteady One-Dimensional Flow", Gas Dynamics - Multidimensional flow, Vol. 2, 1977, pp. 326-328. 